\title{
IMPACT OF RESERVOIR-DEVELOPMENT ALTERNATIVES ON STREAMFLOW QUANTITY IN THE YAMPA RIVER BASIN, COLORADO AND WYOMING
}

\section{U. S. GEOLOGICAL SURVEY}

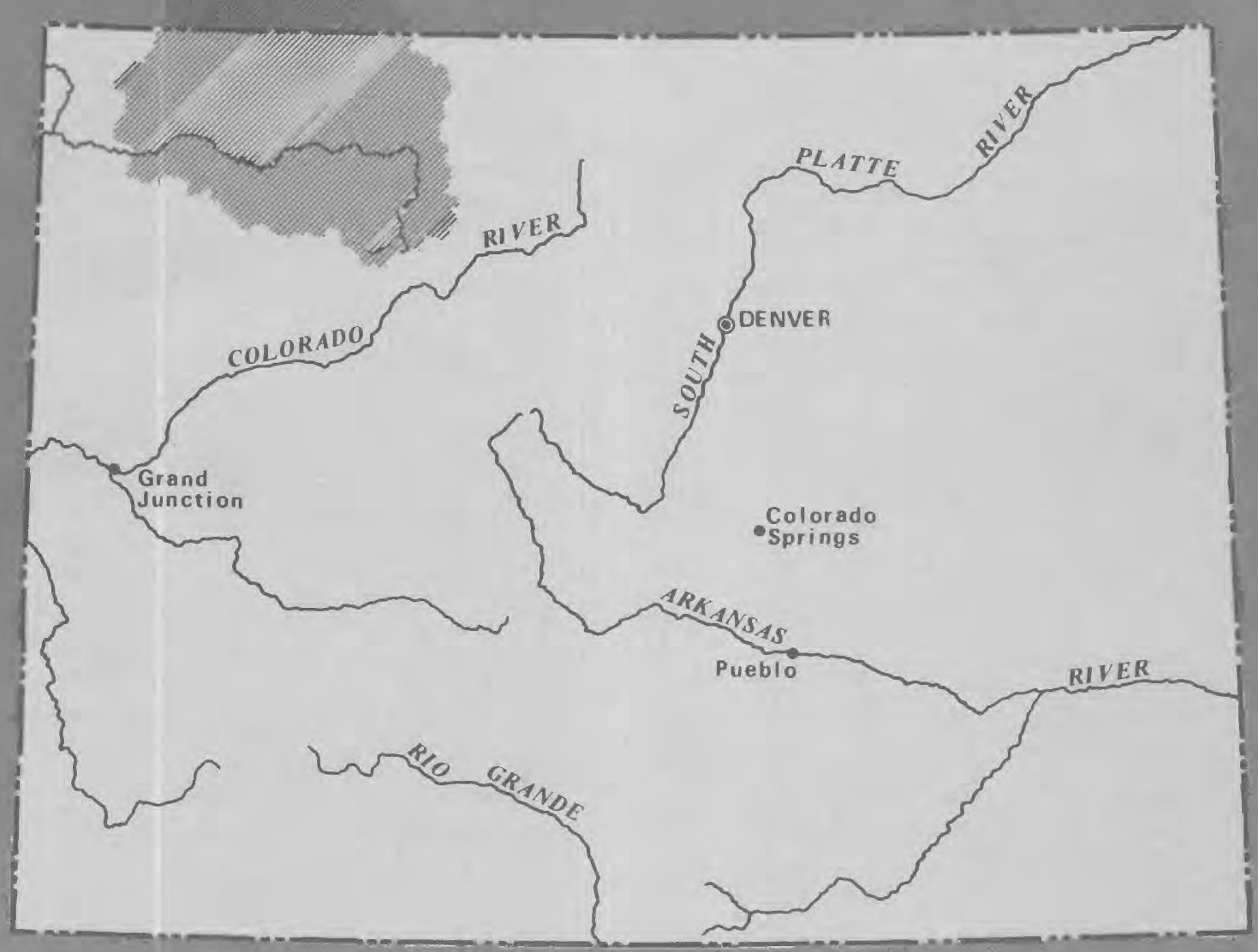

Water-Resources Investigations 80-113

Prepared in cooperation with the U.S. Fish and Wildlife Service 


\begin{tabular}{|c|c|c|c|}
\hline $\begin{array}{l}\text { REPORT DOCUMENTATION } \\
\text { PAGE }\end{array}$ & 1. REPORT & No. & 3. Reciplent's Accession No. \\
\hline \multirow{3}{*}{\multicolumn{3}{|c|}{$\begin{array}{l}\text { 4. Title and subtitle } \\
\text { IMPACT OF RESERVOIR-DEVELOPMENT ALTERNATIVES ON STREAMFLOW } \\
\text { QUANTITY IN THE YAMPA RIVER BASIN, COLORADO AND WYOMING }\end{array}$}} & 5. Report Date \\
\hline & & & March 1982 \\
\hline & & & 6. \\
\hline \multicolumn{3}{|c|}{$\begin{array}{l}\text { 7. Author(s) } \\
\text { Jack E. Veenhuis and Donald E. Hillier }\end{array}$} & $\begin{array}{l}\text { 8. Performing Organization Rept. No. } \\
\text { USGS/WRI } 80-113\end{array}$ \\
\hline \multirow{2}{*}{\multicolumn{3}{|c|}{ 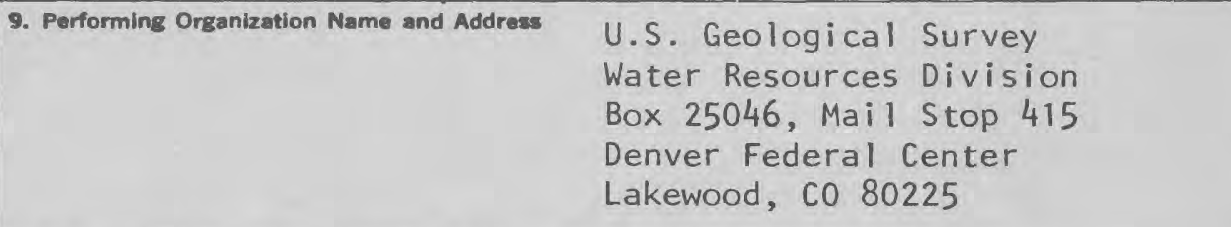 }} & 10. Project/Tsak/Work Unit No. \\
\hline & & & $\begin{array}{l}\text { 11. Contract(C) or Grant(G) No. } \\
\text { (C) } \\
\text { (G) }\end{array}$ \\
\hline \multirow{2}{*}{\multicolumn{3}{|c|}{$\begin{array}{l}\text { 12. Sponsoring Organization Name and Address } \\
\text { U.S. Geological Survey } \\
\text { Water Resources Division } \\
\text { Box 25046, Mail Stop } 415 \\
\text { Denver Federal Center } \\
\text { Lakewood Co } 80225\end{array}$}} & 13. Type of Report \& Period Covered \\
\hline & & & 14. \\
\hline
\end{tabular}

15. Supplementary Notes Lakewood, co 80225

16. Abstract (Limit: 200 words) In the Yampa River basin, Colorado-Wyoming, 35 major reservoirs and 2 transmountain diversions have been proposed to provide additional water for increasing uses. A multireservoir-flow computer model was used to simulate effects on streamflow of five potential options--one representing historical conditions and four representing various degrees of reservoir and transmountain-diversion development. Various combinations of 17 proposed reservoirs and 2 transmountain diversions were used. Varying percentages (25, 50, 75, and 100 percent) of hypothetical agricultural and transmountain diversions within each proposed reservoir-development option were simulated for different degrees of water use, providing results for alternatives.

The proposed Vidler transmountain diversion would affect streamflow only in the Yampa River subbasin while the proposed addition to the Hog Park transmountain diversion would affect streamflow primarily in the Little Snake River subbasin. Streamflow in the Yampa River tributaries could be relatively unaffected by the Vidler transmountain diversion, although streamflow could be affected to some degree in all reaches of the Yampa River downstream from the proposed diversion site.

More uniform flow regimens during the year could result from some proposed options. However, existing (1979) minimum streamflows would not be maintained in many instances, and for many months, with the larger percentage of water-use allocation, there could be no streamflow.

17. Document Analysis a. Descriptors

Reservoir simulation, Reservoir development, Routing, Simulation, Computer model, Minimum flow, Instream flow

b. Identifiers/Open-Ended Terms

Yampa River, Little Snake River, Colorado

c. COSATI Field/Group

18. Availability Statemen:

No restriction on distribution

19. Security Class (This Report)

20. Security Class (This Page)
21. No. of Pages

81

22. Price 


\section{IMPACT OF RESERVOIR-DEVELOPMENT ALTERNATIVES ON STREAMFLOW}

QUANTITY IN THE YAMPA RIVER BASIN, COLORADO AND WYOMING

By Jack E. Veenhuis and Donald E. Hillier

U.S. GEOLOGICAL SURVEY

Water-Resources Investigations 80-113

Prepared in cooperation wi th the

U.S. FISH AND WILDLIFE SERVICE

Lakewood, Colorado

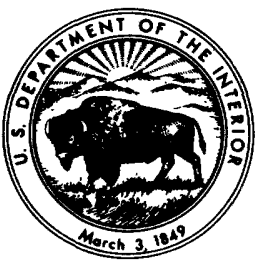

1982 


\section{UNITED STATES DEPARTMENT OF THE INTERIOR}

JAMES G. WATT, Secretary

GEOLOGICAL SURVEY

Dallas L. Peck, Director

For additional information write to:

Colorado District Chief, WRD

U.S. Geological Survey

Box 25046, Mail Stop 415

Denver Federal Center

Lakewood, C0 80225 


\section{CONTENTS}

Abstract-

Introduction-

Model description-

Data availability

Streamflow records-

Precipitation records-

Evaporation records-

Consumptive use and existing surface-water diversions-1.-

Reservoir geometry-

Alternative reservoir configurations studied-

Proposed diversions used in the model-

Agricultural diversions

Industrial and municipal diversions-

Transmountain diversions-_.

Model verification-

Model simulations

Model results-

Summary-

Selected references-

\section{ILLUSTRATIONS}

Figure 1. Map showing location of some representative proposed reservoirs and control points used in multireservoir-modeling analysis----

2. Map showing location of streamflow-gaging and climatological stations having records used in multireservoir-modeling analysis-.....

3. Graph showing periods of record for streamflow data used in the multireservoir-modeling analysis--

4-6. Graphs showing simulated and measured mean annual streamflow at: 4. Control point 39, Yampa River at Steamboat Springs, Colo., 1927-76 water years-1.-1

5. Control point 18, Yampa River near Maybell, Colo., 1927-76 water years-16 16

6. Control point 42, Little Snake River near Lily, Colo., 1927-76 water years-1.

\section{TABLES}

Table 1. Monthly evaporation rates used for reservoirs in the Yampa River basin-.......

2. Assumed monthly schedules for proposed and existing agricultural diversions-.-.

3. Proposed reservoirs used in model analysis- 
Tables:

4. Proposed and existing monthly diversions for industrial and municipal use--...-

5. Assumed monthly schedules for transmountain diversions-_...........

6. Control points for which results of model simulations are presented-

7. Summary of monthly streamflows, control point 39 (Yampa River at Steamboat Springs, Colo.), for simulated historical conditions, including 100 percent of transmountain diversions, and for historical conditions

8-11. Summary of simulated historical monthly streamflows, control point 33 (Yampa River at Steamboat Springs, Colo.):

8. With 25 percent of agricultural and no transmountain diversions, and with 25 percent of both agricultural and transmountain diversions, and including 100 percent of industrial and municipal diversions for all simulations--

9. With 50 percent of agricultural and no transmountain diversions, and with 50 percent of both agricultural and transmountain diversions, and including 100 percent of industrial and municipal diversions for all simulations--

10. With 75 percent of agricultural and no transmountain diversions, and with 75 percent of both agricultural and transmountain diversions, and including 100 percent of industrial and municipal diversions for all simulations--

11. With 100 percent of agricultural and no transmountain diversions, and with 100 percent of both agricultural and transmountain diversions, and including 100 percent of industrial and municipal diversions for all simulations--

12. Summary of monthly streamflows, control point 38 (Elk River near Trull, Colo.), for simulated historical conditions, including 100 percent of transmountain diversions, and for historical conditions

13-16. Summary of simulated historical monthly streamflows, control point 38 (Elk River near Trull, Colo.):

13. With 25 percent of agricultural and no transmountain diversions, and with 25 percent of both agricultural and transmountain diversions, and including 100 percent of industrial and municipal diversions for all simulations--

14. With 50 percent of agricultural and no transmountain diversions, and with 50 percent of both agricultural and transmountain diversions, and including 100 percent of industrial and municipal diversions for all simulations--

15. With 75 percent of agricultural and no transmountain diversions, and with 75 percent of both agricultural and transmountain diversions, and including 100 percent of industrial and municipal diversions for all simulations--

16. With 100 percent of agricultural and no transmountain diversions, and with 100 percent of both agricultural and transmountain diversions, and including 100 percent of industrial and municipal diversions for all simulations-- 


\section{CONTENTS--Continued}

Tables:

Page

17-21. Summary of simulated historical monthly streamflows, control

point 34 (Trout Creek at mouth):

17. For historical conditions and with 100 percent of transmountain diversions-...

18. With 25 percent of agricultural and no transmountain diversions, and with 25 percent of both agricultural and transmountain diversions, and including 100 percent of industrial and municipal diversions for all simulations--

19. With 50 percent of agricultural and no transmountain diversions, and with 50 percent of both agricultural and transmountain diversions, and including 100 percent of industrial and municipal diversions for all simulations--

20. With 75 percent of agricultural and no transmountain diversions, and with 75 percent of both agricultural and transmountain diversions, and including 100 percent of industrial and municipal diversions for all simulations--

21. With 100 percent of agricultural and no transmountain diversions, and with 100 percent of both agricultural and transmountain diversions, and including 100 percent of industrial and municipal diversions for all simulations--

22-26. Summary of simulated historical monthly streamflows, control point 15 (Yampa River at Craig, Colo.):

22. For historical conditions and with 100 percent of trans-

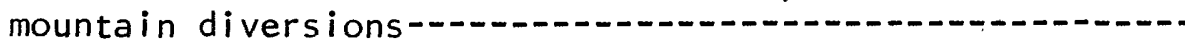

23. With 25 percent of agricultural and no transmountain diversions, and with 25 percent of both agricultural and transmountain diversions, and including 100 percent of industrial and municipal diversions for all simulations--

24. With 50 percent of agricultural and no transmountain diversions, and with 50 percent of both agricultural and transmountain diversions, and including 100 percent of industrial and municipal diversions for all simulations--

25. With 75 percent of agricultural and no transmountain diversions, and with 75 percent of both agricultural and transmountain diversions, and including 100 percent of industrial and municipal diversions for all simulations--

26. With 100 percent of agricultural and no transmountain diversions, and with 100 percent of both agricultural and transmountain diversions, and including 100 percent of industrial and municipal diversions for all simulations--

27-31. Summary of simulated historical monthly streamflows, control point 25 (confluence of Yampa River and Milk Creek):

27. For simulated historical conditions and with 100 percent of transmountain diversions-

28. With 25 percent of agricultural and no transmountain diversions, and with 25 percent of both agricultural and transmountain diversions, and including 100 percent of industrial and municipal diversions for all simulations-- 
Tables:

27-31. Summary of simulated historical monthly streamflows, control point 25 (confluence of Yampa River and Milk Creek)--Continued:

29. With 50 percent of agricultural and no transmountain diversions, and with 50 percent of both agricultural and transmountain diversions, and including 100 percent of industrial and municipal diversions for all simulations--

30. With 75 percent of agricultural and no transmountain diversions, and with 75 percent of both agricultural and transmountain diversions, and including 100 percent of industrial and municipal diversions for all simulations--

31. With 100 percent of agricultural and no transmountain

diversions, and with 100 percent of both agricultural and transmountain diversions, and including 100 percent of industrial and municipal diversions for all simulations--

32. Summary of monthly streamflows, control point 18 (Yampa River near Maybell, Colo.), for simulated historical conditions, including 100 percent of transmountain diversions, and for historical conditions--.-

33-36. Summary of simulated historical monthly streamflows, control point 18 (Yampa River near Maybell, Colo.):

33. With 25 percent of agricultural and no transmountain diversions, and with 25 percent of both agricultural and transmountain diversions, and including 100 percent of industrial and municipal diversions for all simulations--

34. With 50 percent of agricultural and no transmountain diversions, and with 50 percent of both agricultural and transmountain diversions, and including 100 percent of industrial and municipal diversions for all simulations--

35. With 75 percent of agricultural and no transmountain diversions, and with 75 percent of both agricultural and transmountain diversions, and including 100 percent of industrial and municipal diversions for all simulations--

36. With 100 percent of agricultural and no transmountain diversions, and with 100 percent of both agricultural and transmountain diversions, and including 100 percent of industrial and municipal diversions for all simulations--

37-41. Summary of simulated historical monthly streamflows, control point 19 (Yampa River near Lily, Colo.):

37. For historical conditions and with 100 percent of transmountain diversions

38. With 25 percent of agricultural and no transmountain diversions, and with 25 percent of both agricultural and transmountain diversions, and including 100 percent of industrial and municipal diversions for all simulations-ith 50 percent of agricultural and no transmountain diversions, and with 50 percent of both agricultural and transmountain diversions, and including 100 percent of industrial and municipal diversions for all simulations-- 
Tables

37-41. Summary of simulated historical monthly streamflows, control

point 19 (Yampa River near Lily, Colo.)--Continued:
40. With 75 percent of agricultural and no transmountain
diversions, and with 75 percent of both agricultural and
transmountain diversions, and including 100 percent of
industrial and municipal diversions for all simulations--
41. With 100 percent of agricultural and no transmountain
diversions, and with 100 percent of both agricultural and
transmountain diversions, and including 100 percent of
industrial and municipal diversions for all simulations--

point 19 (Yampa River near Lily, Colo.)--Continued:
40. With 75 percent of agricultural and no transmountain
diversions, and with 75 percent of both agricultural and
transmountain diversions, and including 100 percent of
industrial and municipal diversions for all simulations--
41. With 100 percent of agricultural and no transmountain
diversions, and with 100 percent of both agricultural and
transmountain diversions, and including 100 percent of
industrial and municipal diversions for all simulations--

point 19 (Yampa River near Lily, Colo.)--Continued:
40. With 75 percent of agricultural and no transmountain
diversions, and with 75 percent of both agricultural and
transmountain diversions, and including 100 percent of
industrial and municipal diversions for all simulations--
41. With 100 percent of agricultural and no transmountain
diversions, and with 100 percent of both agricultural and
transmountain diversions, and including 100 percent of
industrial and municipal diversions for all simulations--

point 19 (Yampa River near Lily, Colo.)--Continued:
40. With 75 percent of agricultural and no transmountain
diversions, and with 75 percent of both agricultural and
transmountain diversions, and including 100 percent of
industrial and municipal diversions for all simulations--
41. With 100 percent of agricultural and no transmountain
diversions, and with 100 percent of both agricultural and
transmountain diversions, and including 100 percent of
industrial and municipal diversions for all simulations--

point 19 (Yampa River near Lily, Colo.)--Continued:
40. With 75 percent of agricultural and no transmountain
diversions, and with 75 percent of both agricultural and
transmountain diversions, and including 100 percent of
industrial and municipal diversions for all simulations--
41. With 100 percent of agricultural and no transmountain
diversions, and with 100 percent of both agricultural and
transmountain diversions, and including 100 percent of
industrial and municipal diversions for all simulations--

point 19 (Yampa River near Lily, Colo.)--Continued:
40. With 75 percent of agricultural and no transmountain
diversions, and with 75 percent of both agricultural and
transmountain diversions, and including 100 percent of
industrial and municipal diversions for all simulations--
41. With 100 percent of agricultural and no transmountain
diversions, and with 100 percent of both agricultural and
transmountain diversions, and including 100 percent of
industrial and municipal diversions for all simulations--

point 19 (Yampa River near Lily, Colo.)--Continued:
40. With 75 percent of agricultural and no transmountain
diversions, and with 75 percent of both agricultural and
transmountain diversions, and including 100 percent of
industrial and municipal diversions for all simulations--
41. With 100 percent of agricultural and no transmountain
diversions, and with 100 percent of both agricultural and
transmountain diversions, and including 100 percent of
industrial and municipal diversions for all simulations--

point 19 (Yampa River near Lily, Colo.)--Continued:
40. With 75 percent of agricultural and no transmountain
diversions, and with 75 percent of both agricultural and
transmountain diversions, and including 100 percent of
industrial and municipal diversions for all simulations--
41. With 100 percent of agricultural and no transmountain
diversions, and with 100 percent of both agricultural and
transmountain diversions, and including 100 percent of
industrial and municipal diversions for all simulations--

point 19 (Yampa River near Lily, Colo.)--Continued:
40. With 75 percent of agricultural and no transmountain
diversions, and with 75 percent of both agricultural and
transmountain diversions, and including 100 percent of
industrial and municipal diversions for all simulations--
41. With 100 percent of agricultural and no transmountain
diversions, and with 100 percent of both agricultural and
transmountain diversions, and including 100 percent of
industrial and municipal diversions for all simulations-near Baggs, Wyo.), for simulated historical conditions, including 100 percent of transmountain diversions, and for historical conditions--.-_-

43-46. Summary of simulated historical monthly streamflows, control point 43 (Little Snake River near Baggs, Wyo.):

43. With 25 percent of agricultural and no transmountain diversions, and with 25 percent of both agricultural and transmountain diversions, and including 100 percent of industrial and municipal diversions for all simulations--

44. With 50 percent of agricultural and no transmountain diversions, and with 50 percent of both agricultural and transmountain diversions, and including 100 percent of industrial and municipal diversions for all simulations--

45. With 75 percent of agricultural and no transmountain diversions, and with 75 percent of both agricultural and transmountain diversions, and including 100 percent of industrial and municipal diversions for all simulations--

46. With 100 percent of agricultural and no transmountain diversions, and with 100 percent of both agricultural and transmountain diversions, and including 100 percent of industrial and municipal diversions for all simulations--

47-51. Summary of simulated historical monthly streamflows, control point 41 (Yampa River near Deerlodge Park, Colo.):

47. For historical conditions and with 100 percent of trans-

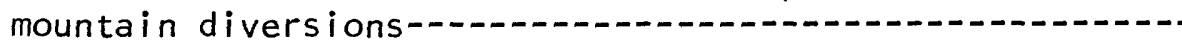

48. With 25 percent of agricultural and no transmountain diversions, and with 25 percent of both agricultural and transmountain diversions, and including 100 percent of industrial and municipal diversions for all simulations--

49. With 50 percent of agricultural and no transmountain
diversions, and with 50 percent of both agricultural and transmountain diversions, and including 100 percent of industrial and municipal diversions for all simulations-- 
Tables:

47-51. Summary of simulated historical monthly streamflows, control

point 41 (Yampa River near Deerlodge Park, Colo.)--Continued:

50. With 75 percent of agricultural and no transmountain diversions, and with 75 percent of both agricultural and transmountain diversions, and including 100 percent of industrial and municipal diversions for all simulations--

51. With 100 percent of agricultural and no transmountain

diversions, and with 100 percent of both agricultural and transmountain diversions, and including 100 percent of industrial and municipal diversions for all simulations--

METRIC CONVERSIONS

inch (in.)

mile (mi)

acre-foot (acre-ft)

cubic foot per second $\left(\mathrm{ft}^{3} / \mathrm{s}\right)$
25.40

1.609

0.001233

0.02832 millimeter

kilometer

cubic hectometer

cubic meter per second 
IMPACT OF RESERVOIR-DEVELOPMENT ALTERNATIVES ON STREAMFLOW QUANTITY

IN THE YAMPA RIVER BASIN, COLORADO AND WYOMING

By Jack E. Veenhuis and Donald E. Hillier

\section{ABSTRACT}

In the Yampa River basin of Colorado and Wyoming, a total of 35 major reservoirs and 2 transmountain diversions has been proposed for construction to provide additional water for increasing industrial, irrigation, and municipal uses. A multireservoir-flow computer model was used to simulate the effects on streamflow of five potential options, including one representing historical conditions and four representing various degrees of reservoir and transmountain-diversion development. Various combinations of 17 proposed reservoirs and the 2 transmountain diversions were used in the analysis. By varying the percentages $(25,50,75$, and 100 percent) of hypothetical agricultural and transmountain diversions within each proposed reservoir-development option studied, different degrees of water-use allocation were simulated, thus providing results for a greater range of alternatives. The results of these simulations provide water managers and planners with some insight into how proposed surface-water developments will affect streamflow.

The proposed Vidler transmountain diversion would affect streamflow only in the Yampa River subbasin while the proposed addition to the Hog Park transmountain diversion would affect streamflow primarily in the Little Snake River subbasin. Streamflow in tributaries to the Yampa River could be relatively unaffected by the Vidler transmountain diversion although streamflow could be affected to some degree in all reaches of the Yampa River downstream from the proposed diversion site.

More uniform flow regimens throughout the year could result from some of the proposed reservoir-development options. However, existing (1979) minimum streamflows would not be maintained in many instances, and for many months with the larger percentage of water-use allocations there could be no streamflow.

\section{INTRODUCTION}

Historically, the principal use of surface water in the Yampa River basin (fig. 1) has been for irrigation of hay meadows and wheat fields. However, increased energy and economic development in the basin will result in additional use of surface water for industrial, municipal, and recreational purposes. Because only 54,000 acre-ft of reservoir storage (Steele and others, 1979) is currently (1979) available in the basin, the construction of numerous reservoirs in the basin has been proposed as a means of providing additional surface water. Proposals include the construction of 35 major reservoirs with a total capacity of $2.18 \mathrm{million}$ acre-ft, which is 41 percent greater than the mean annual outflow 


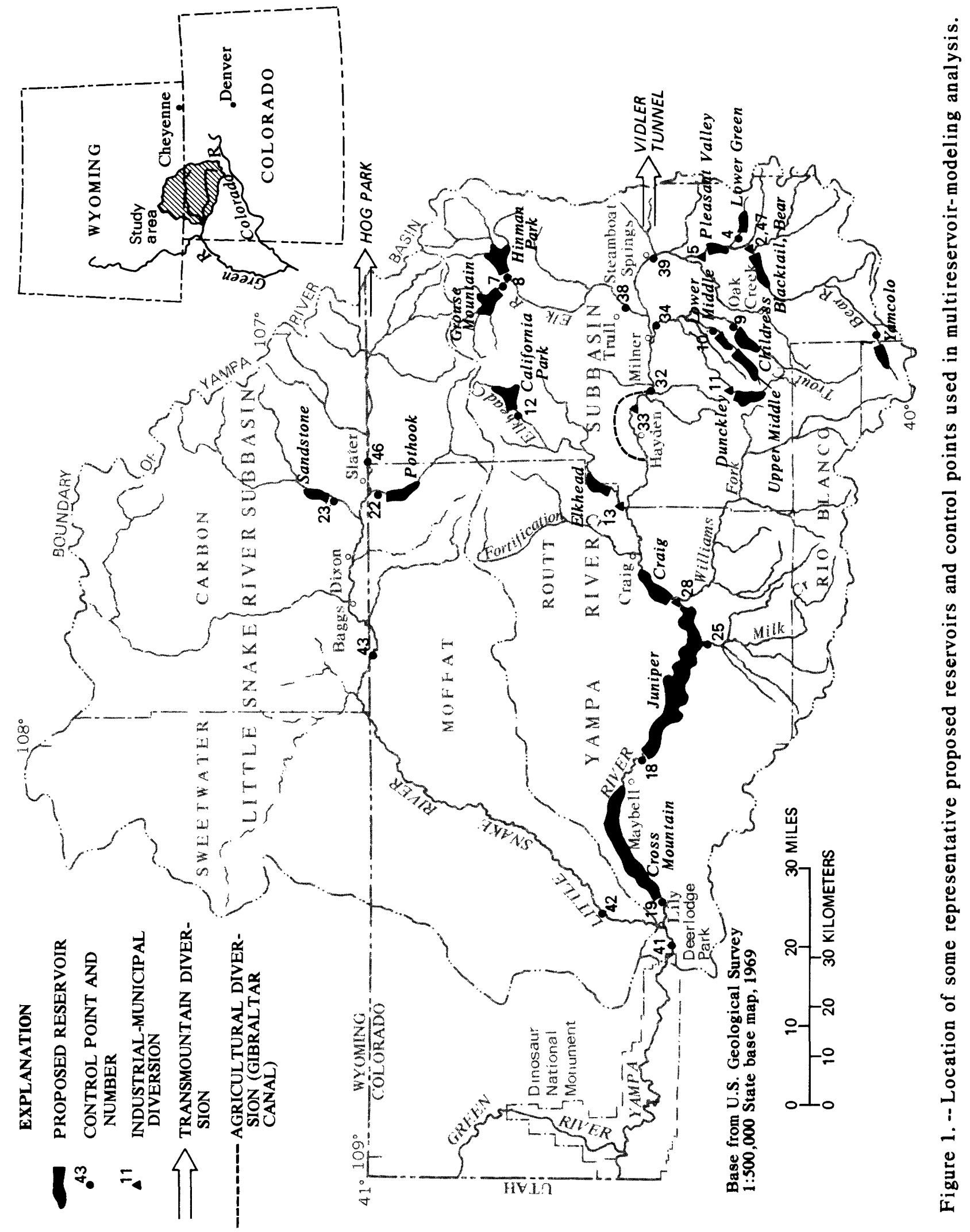


from the basin (Steele and others, 1979). The effects of reservoir development on streamflow and the effects on fish and wildlife habitat need to be determined. Accordingly, the U.S. Fish and Wildlife Service requested that the U.S. Geological Survey determine the effects of potential reservoir configurations and various al locations for irrigation and transmountain diversions on the quantity of streamflow throughout the Yampa River basin.

In this study, a multireservoir-flow model was used to simulate the effects of various configurations of 17 proposed reservoirs, a proposed transmountain diversion, and a proposed addition to a second existing transmountain diversion, on streamflow in the Yampa River basin. The 17 proposed reservoirs are the larger of the total 35 reservoirs being considered for construction in the basin. While the geohydrologic characteristics of the Yampa River basin are well known, the physical characteristics and operating schedules of the reservoirs and transmountain diversions are speculative, as are the flows resulting from the model simulations. To obtain some knowledge of the possible effects on streamflow, five potential options including one representing historical conditions (no additional reservoir development) and four representing various degrees of reservoir and transmountaindiversion development were studied. This study is an extension of earlier reservoir modeling for the Yampa River basin (Adams and others, 1982).

By varying the percentages of agricultural and transmountain diversions within each proposed reservoir-development option studied, different degrees of development were simulated, thus providing results for a greater range of alternatives. The results of these simulations will provide water managers and planners with some insight into how proposed surface-water developments will affect minimum st reamflows.

Results for nine representative control points are presented in this report. Results for the remaining 38 control points may be obtained from the U.S. Geological Survey for the cost of computer and reproduction time.

\section{MODEL DESCRIPTION}

The multireservoir-simulation model used in this study was the HEC-3 multireservoir-flow model developed by the U.S. Army, Corps of Engineers (1968) to do multipurpose, multireservoir routing of streamflow within a river basin. For this study, the Yampa River basin was simulated by 47 control points, arranged in downstream order, representing either a reservoir, a diversion or return-flow point, a confluence of streams, or a stream reach where fish and wildlife habitat is of interest. At all reservoir control points, monthly values were specified for net evaporation (evaporation minus precipitation), downstream discharge-channel capacities, and reservoir geometry (including elevation-area and elevation-volume tables). Storage in each reservoir was divided into six storage and surface-area increments to facilitate approximate simultaneous adjustment of all reservoir levels throughout the basin. Monthly diversions, return flows to the next downstream control point, and estimates of consumptive use were specified at all diversion control points. Between all control points, incremental inflow was computed on the basis of available streamflow records. 


\section{DATA AVAILABILITY}

\section{Streamflow Records}

Daily streamflow records from 36 streamflow-gaging stations, unadjusted for changes in water use (figs. 2 and 3 ), were used to compute mean monthly and mean annual streamflow at the stations for water years 1910-76. Data for periods of no record were synthesized using a least-error, linear-regression technique (A.W. Burns, U.S. Geological Survey, written commun., 1976). Either measured streamflow data or a combination of measured and synthesized streamflow data were used to determine what is termed in this report as "historical conditions" for the modelanalysis period (water years 1927-76). The resulting streamflow data were used to: (1) Determine incremental inflows to proposed reservoirs, and (2) determine incremental inflows between all other control points for the 1927-76 modelanalysis period.

\section{Precipitation Records}

Monthly precipitation records for water years 1910-76 for climatological stations operated by the National Weather Service at Columbine, Craig, Hayden, Pyramid, and Steamboat Springs, Colo., and Dixon, Wyo. (fig. 2), were used in the reservoir analysis. Data for periods of no record were synthesized using a leasterror, linear-regression technique (A. W. Burns, U.S. Geological Survey, written commun., 1976).

\section{Evaporation Records}

Few evaporation data are available for the Yampa River basin. For this reason, monthly evaporation rates determined for reservoirs in the vicinity of Denver, Colo. (Ficke and others, 1976), were used in the reservoir analysis. Monthly evaporation rates for a reservoir in the Yampa River basin were selected from the data in table 1, based on a comparison of geometric characteristics between one of the Denver-vicinity reservoirs and the reservoir of interest in the Yampa River basin. In many instances, the evaporation rates had to be estimated for November through March because ice cover prevented the collection of data (N. E. Spahr, U.S. Geological Survey, written commun., 1977). 


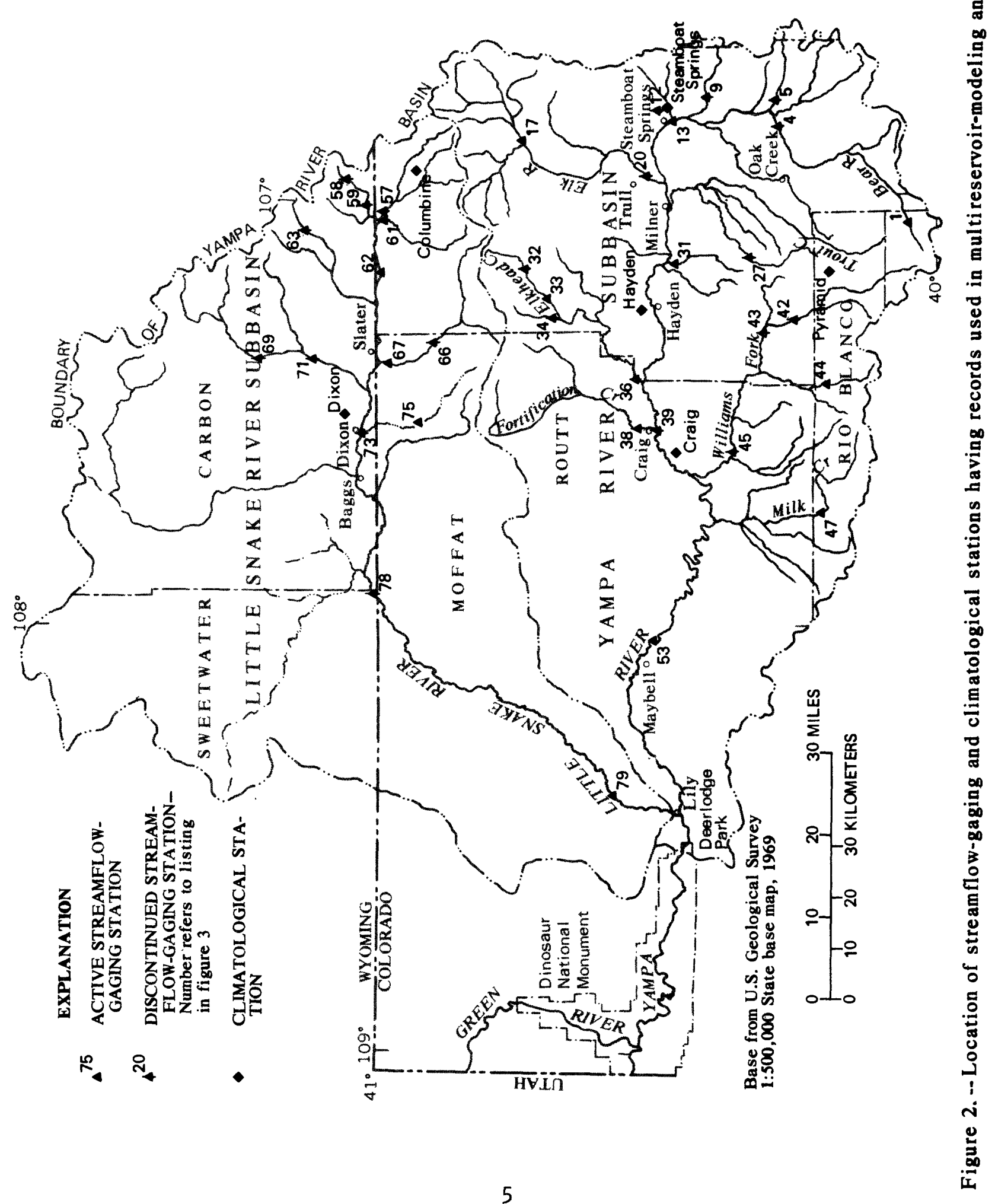




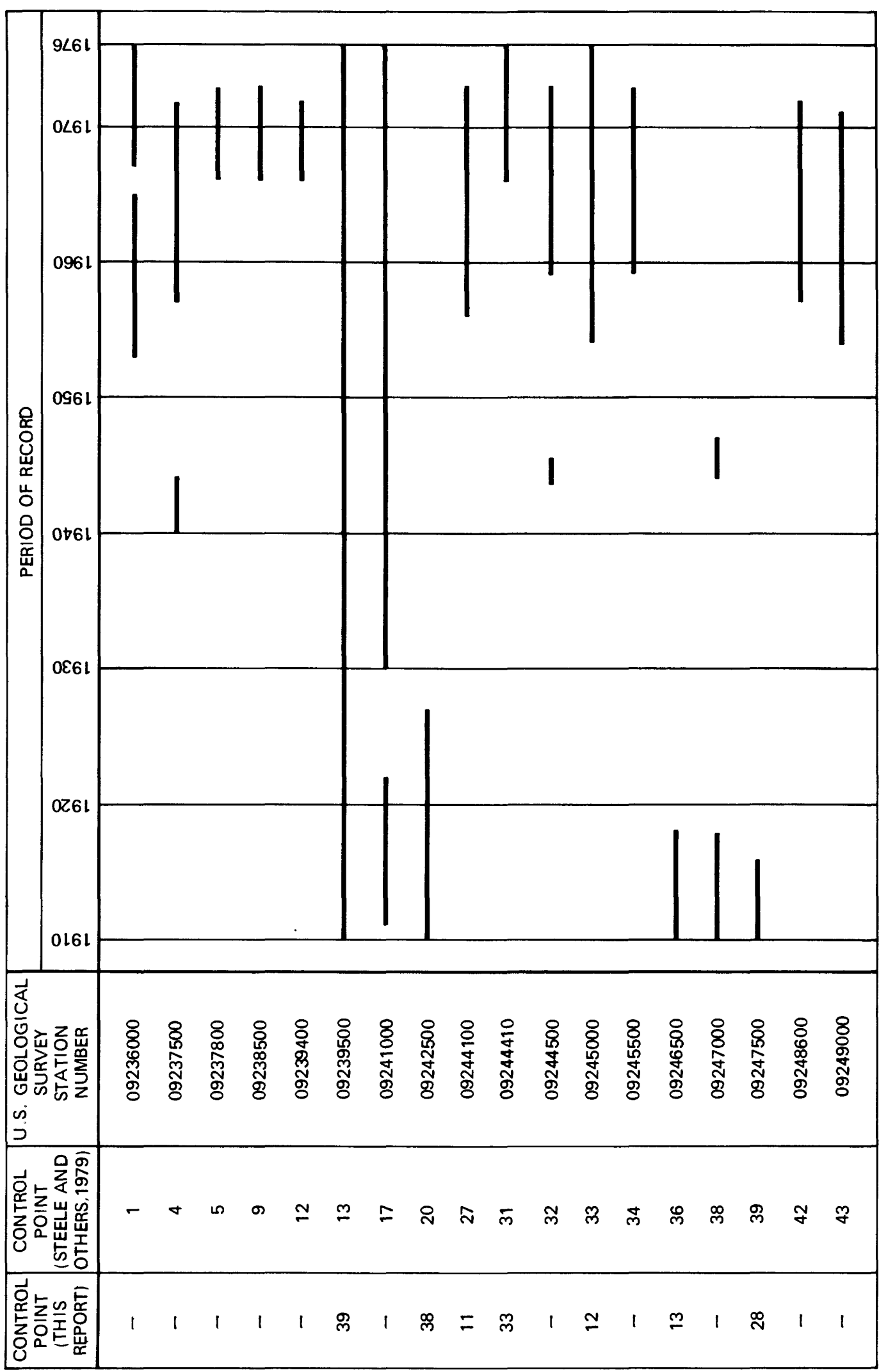

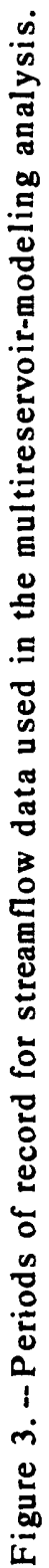




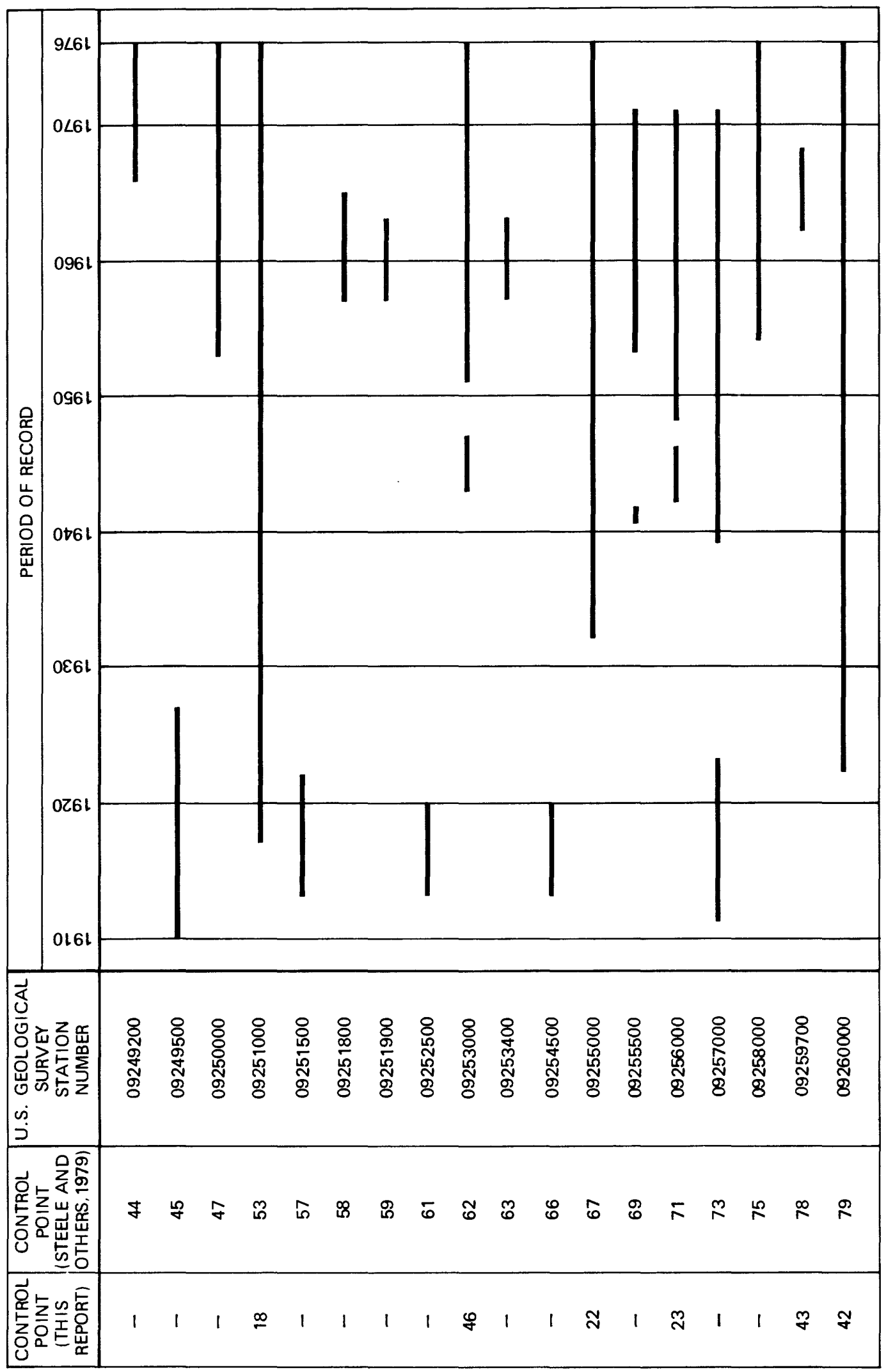

告 


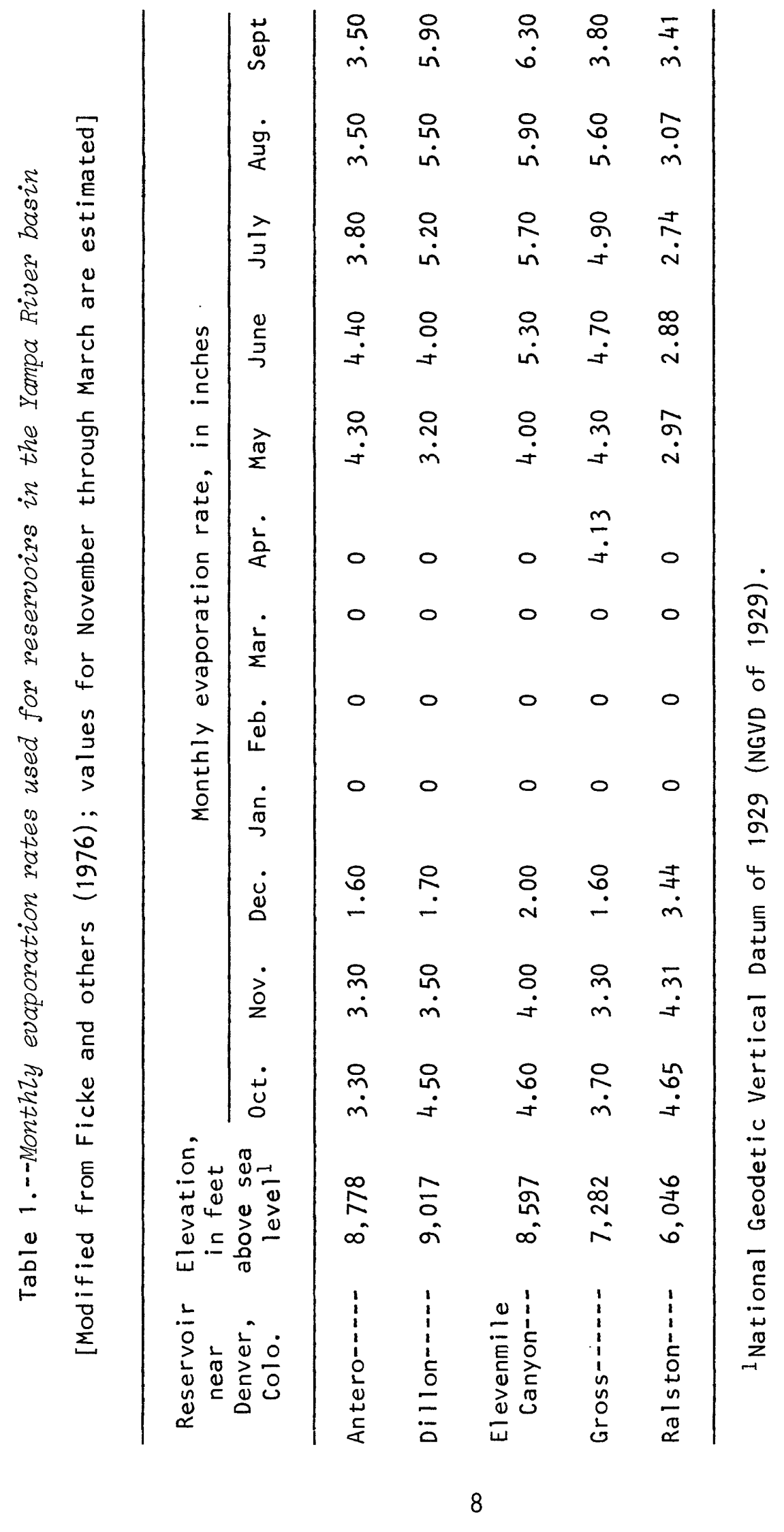




\section{Consumptive Use and Existing Surface-Water Diversions}

Analyses of existing surface-water rights and diversions indicate that more than 90 percent of the water withdrawals and 96 percent of the consumptive use of water in northwestern Colorado during 1976 were attributed to agricultural irrigation (Knudsen and Danielson, 1977; Gray and others, 1977). Most records of diversions to hay and wheat fields and pasturelands in the basin are incomplete. However, incremental inflows between control points accounted for the effects of most of these diversions on streamflow. Diversions through the Gibraltar Canal from the Yampa River near Hayden, Colo., were documented and were included in the reservoir analysis (table 2).

\section{Reservoir Geometry}

Data regarding the geometry of the proposed reservoirs were obtained from Herbert Dishlip (U.S. Bureau of Reclamation, written commun., 1977). Reservoir data obtained included water-surface elevation versus surface area and volume and some preliminary estimates of active storage volumes (conservation pool minus dead storage) for each reservoir. Outflow elevations were generally not available, so estimates were made for dead-storage or conservation-pool elevations. The amount of active storage available for downstream needs was not specified; therefore, for the 100-percent allocation, all available reservoir storage was distributed through the water year. Thus, the 100-percent allocation for each reservoir option represented use of the reservoirs' total active storage volume for diversion purposes.

\section{ALTERNATIVE RESERVOIR CONFIGURATIONS STUDIED}

Because it was not economically feasible to model all possible configurations of the 35 proposed reservoirs, 4 representative reservoir-development options for 17 of the larger proposed reservoirs were chosen as summarized in table 3; the locations of the reservoirs and control points are shown in figure 1 . These options, the same as those used in the U.S. Geological Survey's Yampa River basin assessment, include the largest proportion of the total reservoir storage proposed for the basin (Adams and others, 1982). Using these options, a representative expected range in flow may be simulated for various degrees of reservoir development. 


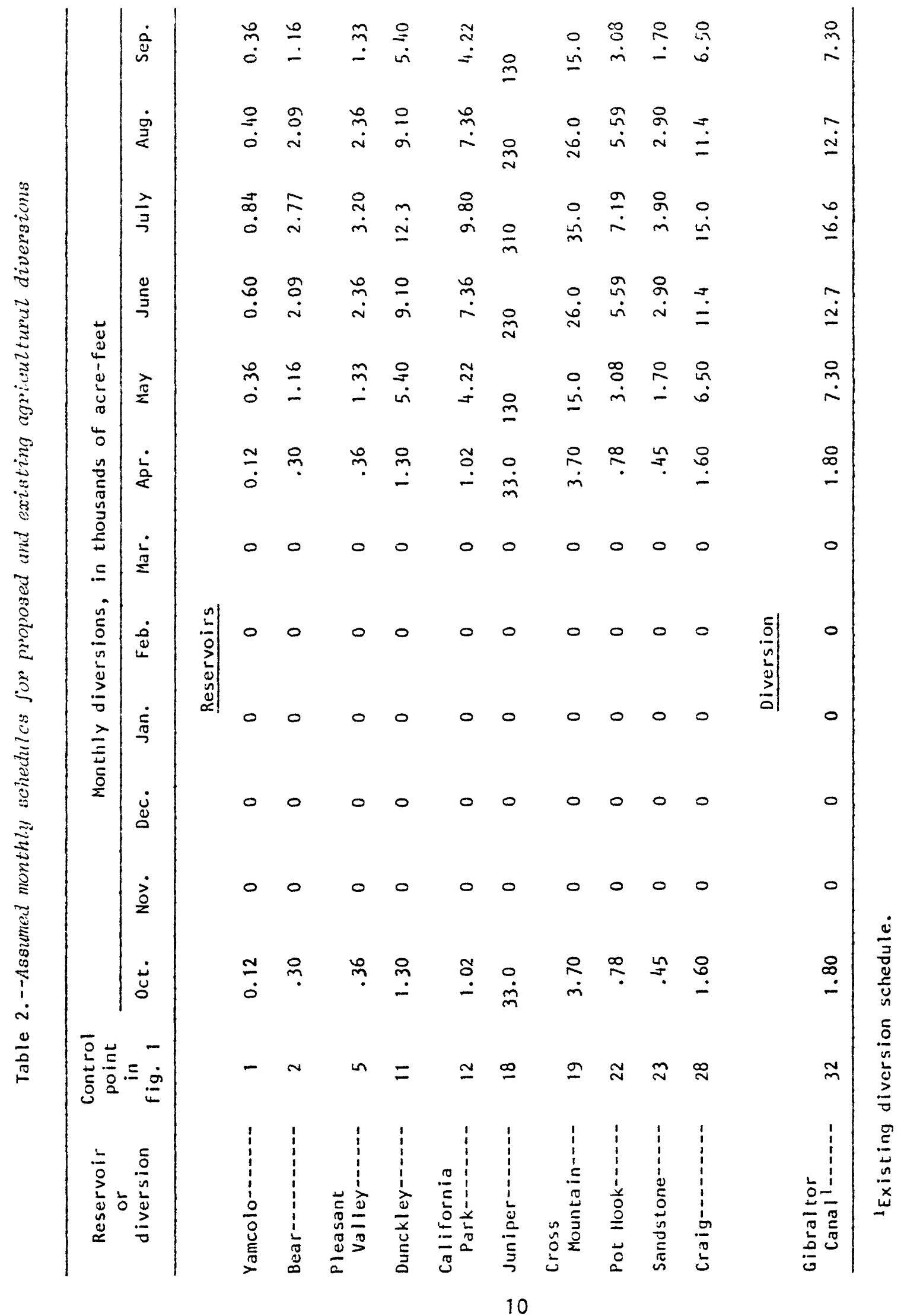


Table 3.--Proposed reservoirs used in model analysis

\begin{tabular}{|c|c|c|c|c|c|c|}
\hline \multirow{2}{*}{ Proposed reservoir } & \multirow{2}{*}{ Stream } & \multirow{2}{*}{$\begin{array}{c}\text { Proposed storage } \\
\text { capacity } \\
\text { (acre-feet) }\end{array}$} & \multicolumn{4}{|c|}{ Option } \\
\hline & & & 1 & 2 & 3 & 4 \\
\hline $\begin{array}{l}\text { Bearl } \\
\text { Cross Mountainl } \\
\text { Juniperl } \\
\text { Yamcolol. } \\
\text { Blacktail }\end{array}$ & $\begin{array}{l}\text { Yampa River } \\
\text { Yampa River } \\
\text { Yampa River } \\
\text { Bear River } \\
\text { Yampa River }\end{array}$ & $\begin{array}{r}11,610 \\
142,000 \\
1,079,990 \\
9,000 \\
229,250\end{array}$ & $\begin{array}{l}x \\
x \\
x \\
x \\
-\end{array}$ & $\begin{array}{l}- \\
x \\
x \\
x \\
x\end{array}$ & $\begin{array}{l}- \\
x \\
x \\
x \\
x\end{array}$ & $\begin{array}{l}- \\
\bar{x} \\
\bar{x}\end{array}$ \\
\hline $\begin{array}{l}\text { Childress } \\
\text { Lower Green- } \\
\text { Lower Middle- } \\
\text { Upper Middle } \\
\text { Pot Hook }\end{array}$ & $\begin{array}{l}\text { Trout Creek } \\
\text { Green Creek } \\
\text { Middle Creek } \\
\text { Middle Creek } \\
\text { Slater Fork }\end{array}$ & $\begin{array}{r}24,160 \\
99,600 \\
25,150 \\
102,200 \\
60,000\end{array}$ & $\begin{array}{l}- \\
- \\
-\end{array}$ & $\begin{array}{l}x \\
x \\
X \\
x \\
X\end{array}$ & $\begin{array}{l}x \\
x \\
x \\
x \\
x\end{array}$ & $\begin{array}{l}x \\
x \\
x \\
x \\
x\end{array}$ \\
\hline $\begin{array}{l}\text { Sandstonel } \\
\text { California Parkl-- } \\
\text { Craigl } \\
\text { Dunckley } \\
\text { Grouse Mountain--- }\end{array}$ & $\begin{array}{l}\text { Savery Creek } \\
\text { Elkhead Creek } \\
\text { Yampa River } \\
\text { Fish Creek } \\
\text { Willow Creek }\end{array}$ & $\begin{array}{l}15,500 \\
36,540 \\
44,490 \\
57,090 \\
79,260\end{array}$ & $\begin{array}{l}- \\
- \\
- \\
-\end{array}$ & $\begin{array}{l}x \\
- \\
- \\
- \\
-\end{array}$ & $\begin{array}{l}x \\
x \\
X \\
x \\
x\end{array}$ & $\begin{array}{l}x \\
x \\
x \\
x \\
x\end{array}$ \\
\hline $\begin{array}{l}\text { Hinman Park- } \\
\text { Pleasant Valley } 1--\end{array}$ & $\begin{array}{l}\text { Elk River } \\
\text { Yampa River }\end{array}$ & $\begin{array}{l}44,040 \\
43,220\end{array}$ & - & $\begin{array}{l}- \\
-\end{array}$ & $\begin{array}{l}x \\
x\end{array}$ & $\begin{array}{l}x \\
x\end{array}$ \\
\hline
\end{tabular}

${ }^{1}$ Proposed diversions for agricultural use.

Some of the proposed larger reservoir complexes considered in this study include: (1) Juniper and Cross Mountain project (Colorado River Water Conservation District, 1975); (2) Oak Creek Water and Power Project (Oak Creek Power Company, 1976), which includes the following proposed reservoirs: Blacktail, Lower Green, Upper and Lower Middle, and Childress; (3) Savery-Pot Hook project (U.S. Department of the Interior, 1976); and (4) Yamcolo project (Western Engineers, Inc., 1975). The proposed Pleasant Valley Reservoir is an expansion of the existing Lake Catamount Reservoir (Woodward-Clyde Consultants, 1977).

\section{PROPOSED DIVERSIONS USED IN THE MODEL}

Diversions associated with reservoir development in the Yampa River basin are proposed for agricultural, industrial, and municipal use within the basin, and municipal use outside the basin (transmountain diversions). In the model simulations, the proposed diversions for agricultural use within the basin were varied by using percentage water-use allocations $(25,50,75$, and 100 percent) of the 
total or part of active reservoir storage used in each option. Proposed diversions for industrial and municipal use within the basin were assumed to be 100-percent usage throughout the analysis; the proposed transmountain diversions also were varied by the same percentages as the proposed diversions for agricultural use.

\section{Agricultural Diversions}

Agricultural diversion for irrigation is one of the largest proposed uses of reservoir storage. An approximate monthly distribution of diversions, most occurring during the growing season, was assumed for all model simulations (table 2). The values shown in table 2 represent 100 percent of the agricultural irrigation water-use allocations from the noted reservoir. For the analysis, it was assumed that the total active reservoir storage was available each year. In the model, it also was assumed that, of the monthly agricultural diversions, two-thirds would be returned to the streams and one-third would be lost--either by plant evapotranspiration or recharge to the ground-water system. Some agricultural diversion control-point locations are shown in figure 1 , but because of the numerous returnflow sites, control points for return flows are not shown in figure 1 .

\section{Industrial and Municipal Diversions}

Proposed industrial and municipal diversions used in the model are listed in table 4 and the corresponding control points are shown in figure 1; the values in table 4 were not varied during the model simulations. It was assumed that industrial diversions would be completely used in the cooling processes associated with electricity generation at fossil-fueled powerplants. Values for the amount of water needed for cooling per megawatt of electricity produced were adapted from computations by Palmer and others (1977). For example, in a wet-cooling tower, 27,000 acre-ft of water is required for every 2,000 megawatts of electricity generated. For municipal uses, it was assumed that one-third of the diversions would be consumed and that two-thirds would be returned to the streams.

\section{Transmountain Diversions}

Two transmountain diversions from the Yampa River basin have been proposed: The Vidler diversion (Sheephorn project) that would divert about 132,000 acre-ft per year from the eastern part of the Yampa River subbasin to the Denver, Colo., metropolitan area (Robert Moreland, Vidler Tunnel Corp., written commun., 1977), and an addition to the existing Hog Park diversion that would divert a total of 31,000 acre-ft per year $(23,000$ acre-ft per year addition to the 8,000 acre-ft per year present diversion) from the eastern part of the Little Snake River subbasin to Cheyenne, Wyo. (Banner \& Associates, Inc., 1976). In the model, control point 39 (Yampa River at Steamboat Springs, Colo.) represents the withdrawal point for the Vidler diversion, which will divert water from the Yampa River and six tributaries upstream from Steamboat Springs, and control point 46 (Little Snake River near Slater, Wyo.) represents the withdrawal point for the expanded Hog Park diversion (fig. 1). The monthly schedules assumed for the diversions, which were based on the availability of water during peak-flow months, are listed in table 5 . 


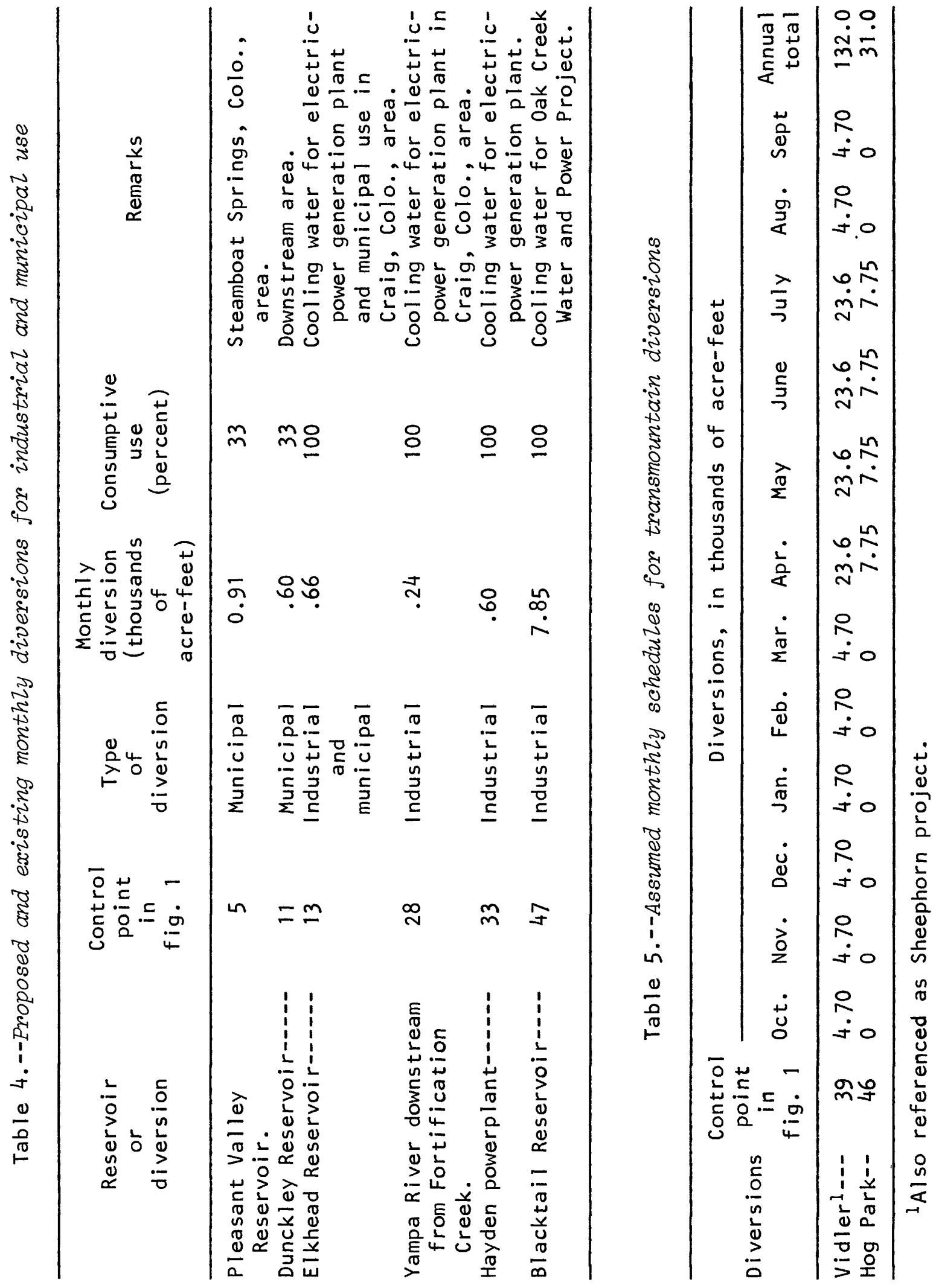




\section{MODEL VERIFICATION}

Because the HEC-3 simulation model has no parameters to calibrate, only verification to gaged streamflow was used to determine the accuracy of its predictive capability for the Yampa River basin. Therefore, a model simulation representing historical conditions with negligible reservoir operations was compared to streamflow records at three streamflow-gaging stations for 50 water years (1927-76). The comparisons between simulated historical and measured mean annual discharges at the three streamflow-gaging stations are shown in figures 4 through 6 . Simulated historical discharges were within 5 percent of measured discharges at control point 39 (Yampa River near Steamboat Springs, Colo.) and control point 42 (Little Snake River near Lily, Colo.), and within 20 percent at control point 18 (Yampa River near Maybell, Colo.). The decrease in accuracy for certain locations is partly due to the uncertainty in accurately representing historical irrigation diversions in the model. On the basis of these simulations, it is concluded that the model has been partly verified for the study area.

\section{MODEL SIMULATIONS}

Because the HEC-3 model is limited to a 50-year interval, model simulations were made for the 50-year period of water years 1927 through 1976. This period was chosen because it included a wide range of climatic conditions, including the droughts of the 1930's and the 1950's.

Thirty-four simulations were made to determine streamflow at the 47 control points in the model. The first simulation determined historical conditions without any proposed transmountain diversions or reservoir development. For the second simulation, the assumption was made that only the two transmountain diversions would be in operation. In each simulation, mean, median, and 80-percent exceedence flows, in cubic feet per second, were determined for each month at each control point. Statistically, median flows for a given month can be expected to be exceeded once every 2 years, on the average, and the 80 -percent exceedence flows can be expected to be exceeded 4 out of every 5 years, on the average.

Simulated historical monthly streamflows at the 47 control points throughout the Yampa River basin were determined as follows:

A. Historical conditions:

1. Historical conditions without any proposed diversions.

2. Historical conditions with 100 percent of proposed transmountain diversions.

B. Reservoir-development options 1 through 4:

1. Allocation of 25 percent of total active reservoir storage for agricultural use without any transmountain diversions, and including 100 percent of industrial and municipal diversions.

2. Allocation of 25 percent of total active reservoir storage for agricultural use with 25 percent of proposed transmountain diversions, and including 100 percent of industrial and municipal diversions. 


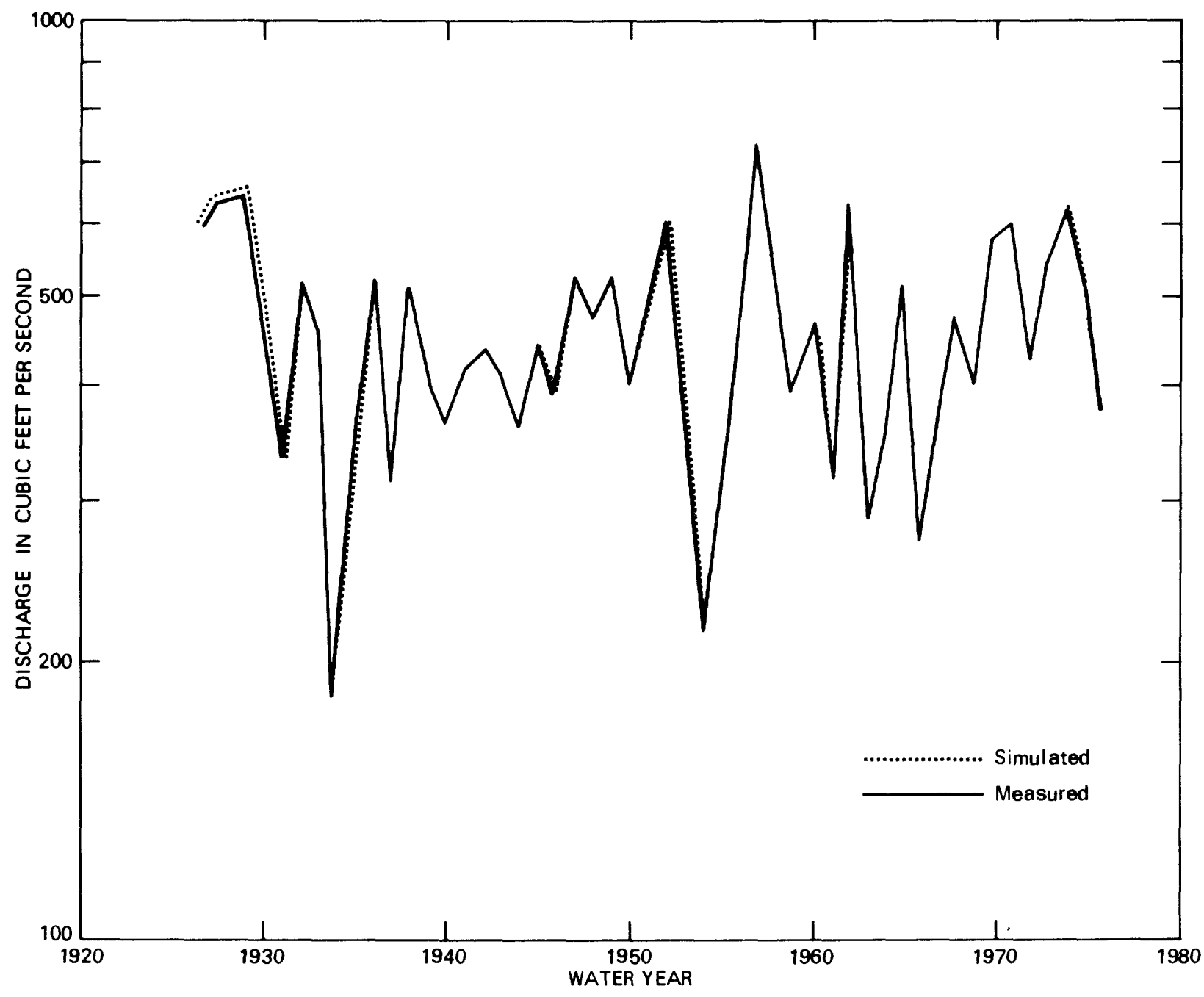

Figure 4. -- Simulated and measured mean annual streamflow at control point 39, Yampa River at Steamboat Springs, Colo., 1927-76 water years. 


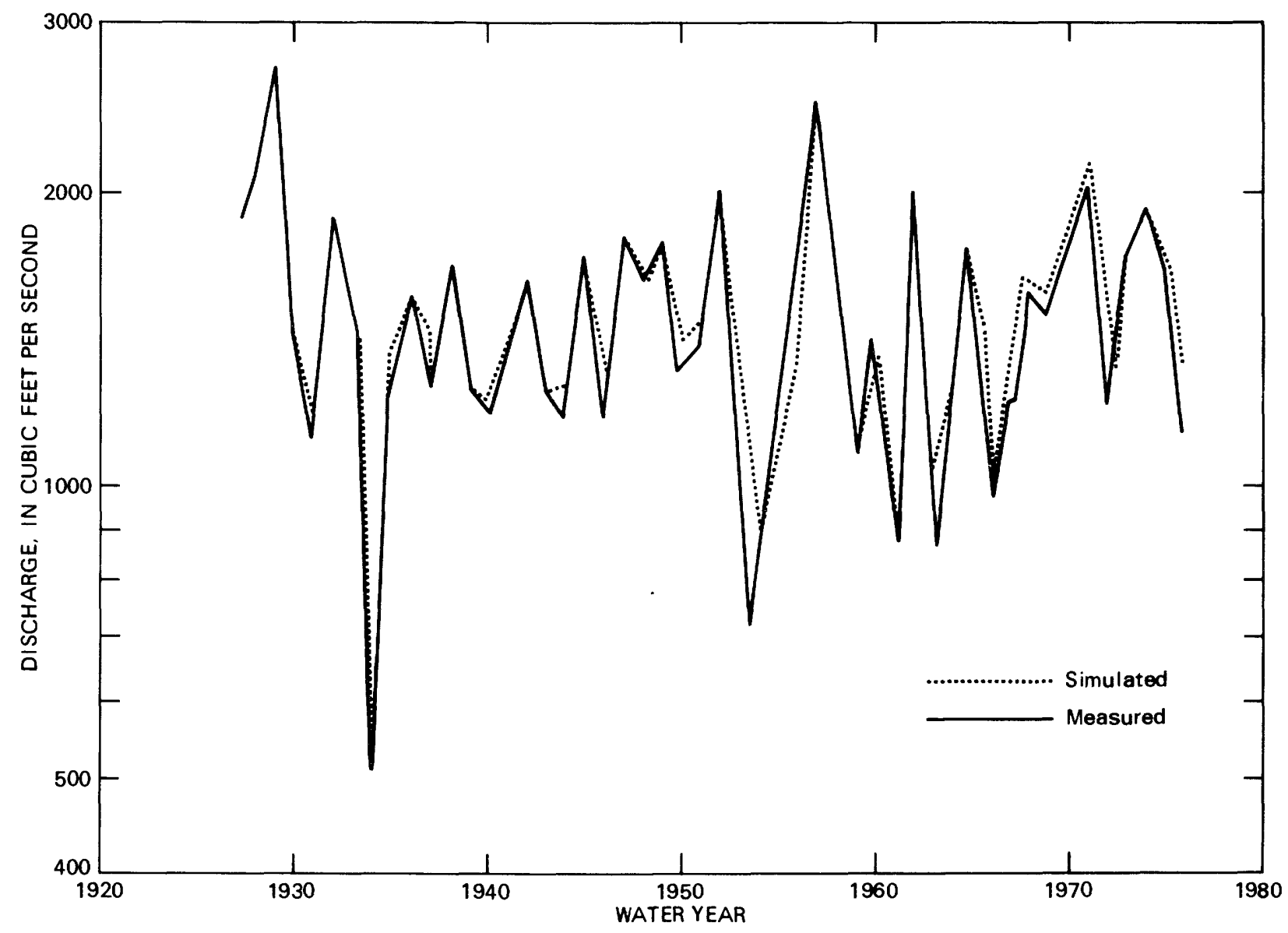

Figure 5. -- Simulated and measured mean annual streamflow at control point 18, Yampa River near Maybell, Colo., 1927-76 water years. 


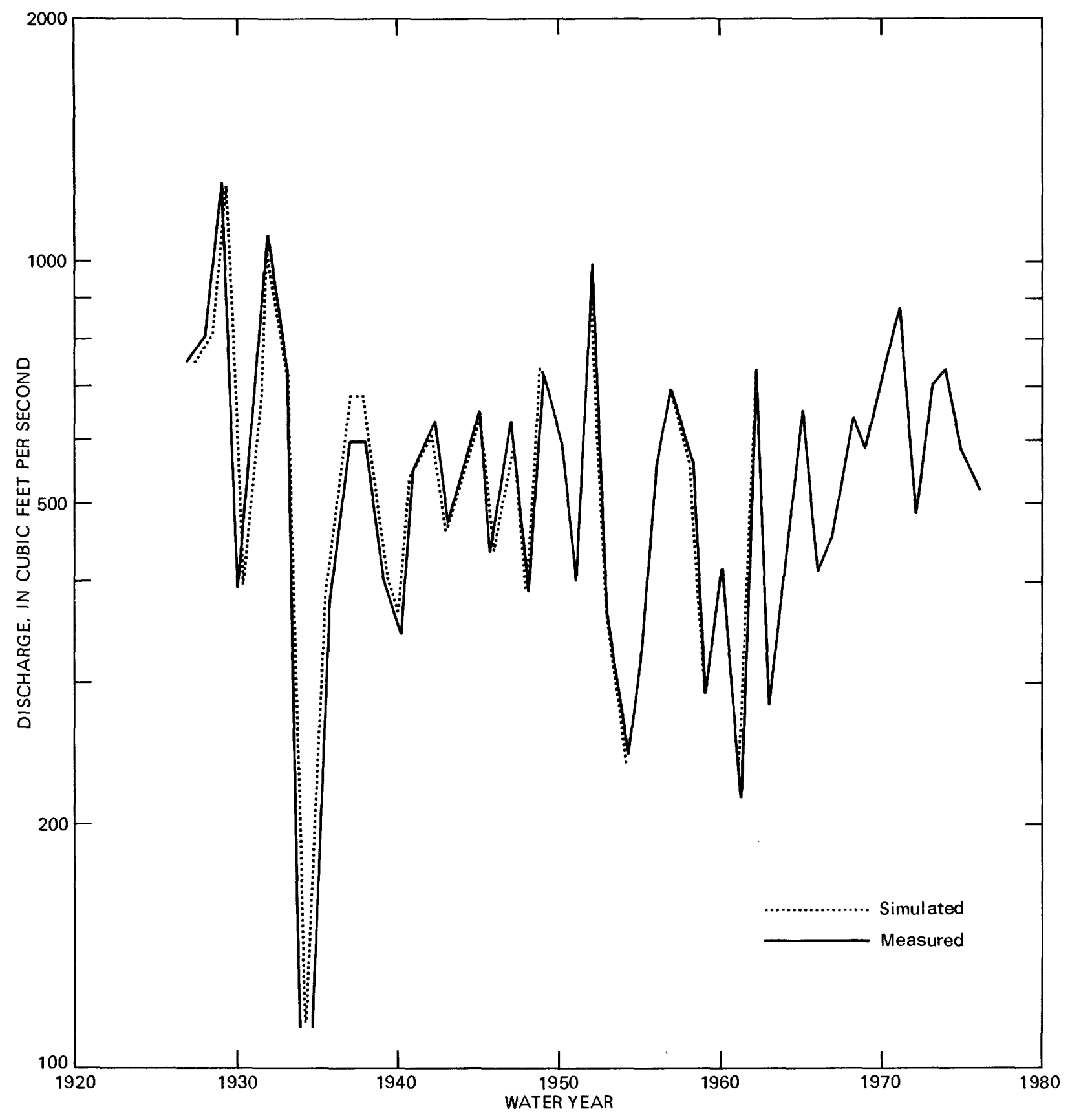

Figure 6. -- Simulated and measured mean annual streamflow at control point 42 , Little Snake River near Lily, Colo., 1927-76 water years. 
3. Allocation of 50 percent of total active reservoir storage for agricultural use without any transmountain diversions, and including 100 percent of industrial and municipal diversions.

4. Allocation of 50 percent of total active reservoir storage for agricultural use with 50 percent of proposed transmountain diversions, and including 100 percent of industrial and municipal diversions.

5. Allocation of 75 percent of total active reservoir storage for agricultural use without any transmountain diversions, and including 100 percent of industrial and municipal diversions.

6. Allocation of 75 percent of total active reservoir storage for agricultural use with 75 percent of proposed transmountain diversions, and including 100 percent of industrial and municipal diversions.

7. Allocation of 100 percent of total active reservoir storage for agricultural use without any transmountain diversions, and including 100 percent of industrial and municipal diversions.

8. Allocation of 100 percent of total active reservoir storage for agricultural use with 100 percent of proposed transmountain diversions, and including 100 percent of industrial and municipal diversions.

\section{MODEL RESULTS}

Results of the model simulations for nine representative control points are presented in this section (table 6). Four of the controls points are at or near streamflow-gaging stations, which permits a comparison with actual conditions in the basin. The model results showing monthly values of mean, median, and 80-percent exceedence flows are presented in five tables for each control point. The first table presents the results of historical conditions with and without transmountain diversions and, where applicable, a summary of the streamflow records for water years 1927-76 from the streamflow-gaging station at or near the control point. The remaining four tables present the results of the $25-, 50-, 75-$, and 100 -percent water-use allocations of the agricultural diversions with and without the transmountain diversions. For all tables, monthly streamflow statistics less than the corresponding values for simulated historical conditions are underscored to indicate reductions in flow. 
Table 6.--Control points for which results of model simulations are presented

\begin{tabular}{|c|c|c|}
\hline $\begin{array}{l}\text { Control } \\
\text { point }\end{array}$ & Location & Significance \\
\hline 39 & $\begin{array}{l}\text { Yampa River at Steamboat Springs, Colo. } \\
\text { (at gaging station 09239500). }\end{array}$ & $\begin{array}{l}\text { Streamflow-gaging-station } \\
\text { control; transmountain } \\
\text { diversion. }\end{array}$ \\
\hline 38 & $\begin{array}{l}\text { Elk River near Trull, Colo. (at gaging } \\
\text { station 09242500). }\end{array}$ & $\begin{array}{l}\text { Streamflow-gaging-station } \\
\text { control; fish habitat. }\end{array}$ \\
\hline 34 & Trout Creek at mouth & Fish habitat. \\
\hline 28 & $\begin{array}{l}\text { Yampa River at Craig, Colo. (down- } \\
\text { stream from proposed Craig } \\
\text { Reservoir). }\end{array}$ & $\begin{array}{l}\text { Industrial and municipal } \\
\text { supplies; fish habitat. }\end{array}$ \\
\hline 25 & $\begin{array}{l}\text { Confluence of Yampa River and } \\
\text { Milk Creek. }\end{array}$ & Fish habitat. \\
\hline 18 & $\begin{array}{l}\text { Yampa River near Maybell, Colo. (at } \\
\text { gaging station 09251000; downstream } \\
\text { from proposed Juniper Reservoir). }\end{array}$ & $\begin{array}{l}\text { Streamflow-gaging-station } \\
\text { control; fish habitat. }\end{array}$ \\
\hline 19 & $\begin{array}{l}\text { Yampa River near Lily, Colo. (down- } \\
\text { stream from proposed Cross Mountain } \\
\text { Reservoir). }\end{array}$ & Fish habitat. \\
\hline 43 & $\begin{array}{l}\text { Little Snake River near Baggs, Wyo. } \\
\text { (near gaging station 09259700). }\end{array}$ & $\begin{array}{l}\text { Streamflow-gaging-station } \\
\text { control; transmountain } \\
\text { diversion. }\end{array}$ \\
\hline 41 & $\begin{array}{l}\text { Yampa River near Deerlodge Park, } \\
\text { Colo. }\end{array}$ & $\begin{array}{l}\text { Commi tments for Upper } \\
\text { Colorado River Basin } \\
\text { Compact. }\end{array}$ \\
\hline
\end{tabular}

Model-simulated historical monthly streamflows for control point 39 (Yampa River at Steamboat Springs, Colo.) are presented in tables 7 through 11. Simulated historical monthly mean streamflows wi thout proposed transmountain diversions vary from +1 to -8 percent and have an average absolute variation of 3 percent of the monthly streamflows calculated from streamflow-gaging-station records, which indicates that the model can reasonably predict conditions at this control point. The average absolute variation is computed by summing the individual absolute values of percentage variations for a given location and model conditions and then dividing by the number of data points. 
Table 7.--Summary of monthly streamflows, control point 39 (Yompa River at Steamboat Springs, Colo.), for simulated historical conditions, including 100 percent of transmountain diversions, and for historical conditions

[FLOW VALUES: $A=M E A N ; B=M E D I A N$; and $C=80$-PERCENT EXCEEDENCE. Underscored values are less than historical conditions without transmountain diversions]

FLOW MONTHLY FLOWS, IN CUBIC FEET PER SECOND

VALUES OCT. NOV. DEC. JAN. FEB. MAR. APR. MAY JUNE JULY AUG. SEPT

\section{SIMULATED HISTORICAL CONDITIONS}

$\begin{array}{rrrrrrrrrrrrr}\text { A } & 130 & 122 & 104 & 100 & 101 & 158 & 669 & 1716 & 1760 & 348 & 145 & 101 \\ \text { B } & 120 & 119 & 102 & 97 & 98 & 144 & 615 & 1565 & 1724 & 276 & 134 & 88 \\ \text { C } & 83 & 97 & 87 & 82 & 83 & 111 & 419 & 1270 & 1128 & 197 & 92 & 69\end{array}$

SIMULATED HISTORICAL CONDITIONS WITH 100 PERCENT OF TRANSMOUNTAIN DIVERSIONS

$$
\begin{array}{lllllllllllll}
\text { A } & \frac{53}{42} & \frac{44}{41} & \frac{27}{24} & \frac{23}{18} & \frac{23}{19} & \frac{80}{66} & \frac{286}{224} & \frac{1325}{1174} & \frac{1373}{1332} & \frac{69}{0} & \frac{69}{56} & \frac{28}{10} \\
\text { C } & \frac{5}{5} & \frac{19}{19} & \frac{1}{9} & \frac{1}{4} & \frac{5}{\underline{5}} & \frac{33}{28} & \frac{879}{\underline{3}} & \frac{737}{14} & \frac{\underline{0}}{0}
\end{array}
$$

HISTORICAL STREAMFLOWS CALCULATED FROM GAGING-STATION RECORDS

\begin{tabular}{rrrrrrrrrrrrr} 
A & 136 & 126 & 104 & 101 & 104 & 172 & 681 & 1771 & 1821 & 345 & 150 & 106 \\
$\mathrm{~B}$ & 132 & 121 & 100 & 100 & 100 & 159 & 630 & 1755 & 1720 & 260 & 136 & 90 \\
$\mathrm{C}$ & 87 & 97 & 87 & 82 & 85 & 115 & 428 & 1288 & 1074 & 163 & 90 & 66 \\
\hline
\end{tabular}

The underscored values in tables 8 through 11 indicate a reduction in the historical flow for any development condition. Only the nonirrigation months of December or January occasionally showed no decrease in flow statistics. Generally, as the reservoir-development options and percentage of water-use allocation increased, the flow volume lessened. Reservoir-development option 4 indicated the most significant reduction in flow as a result of the absence of demand from Juniper and Cross Mountain Reservoirs downstream on the Yampa River. Without the demand from these reservoirs, the flow at this site was reduced and more water remained in the upstream reservoirs. 
Table 8.--Summary of simulated historical monthly streamflows, control point 39 (Yampa River at Steamboat Springs, Colo.), with 25 percent of agricultural and no transmountain diversions, and with 25 percent of both agricultural and transmountain diversions, and including 100 pereent of industrial and municipal diversions for all simulations

[FLOW VALUES: $A=$ MEAN; $B=$ MEDIAN; and $C=80-P E R C E N T$ EXCEEDENCE. Underscored values are less than corresponding table 7 historical conditions]

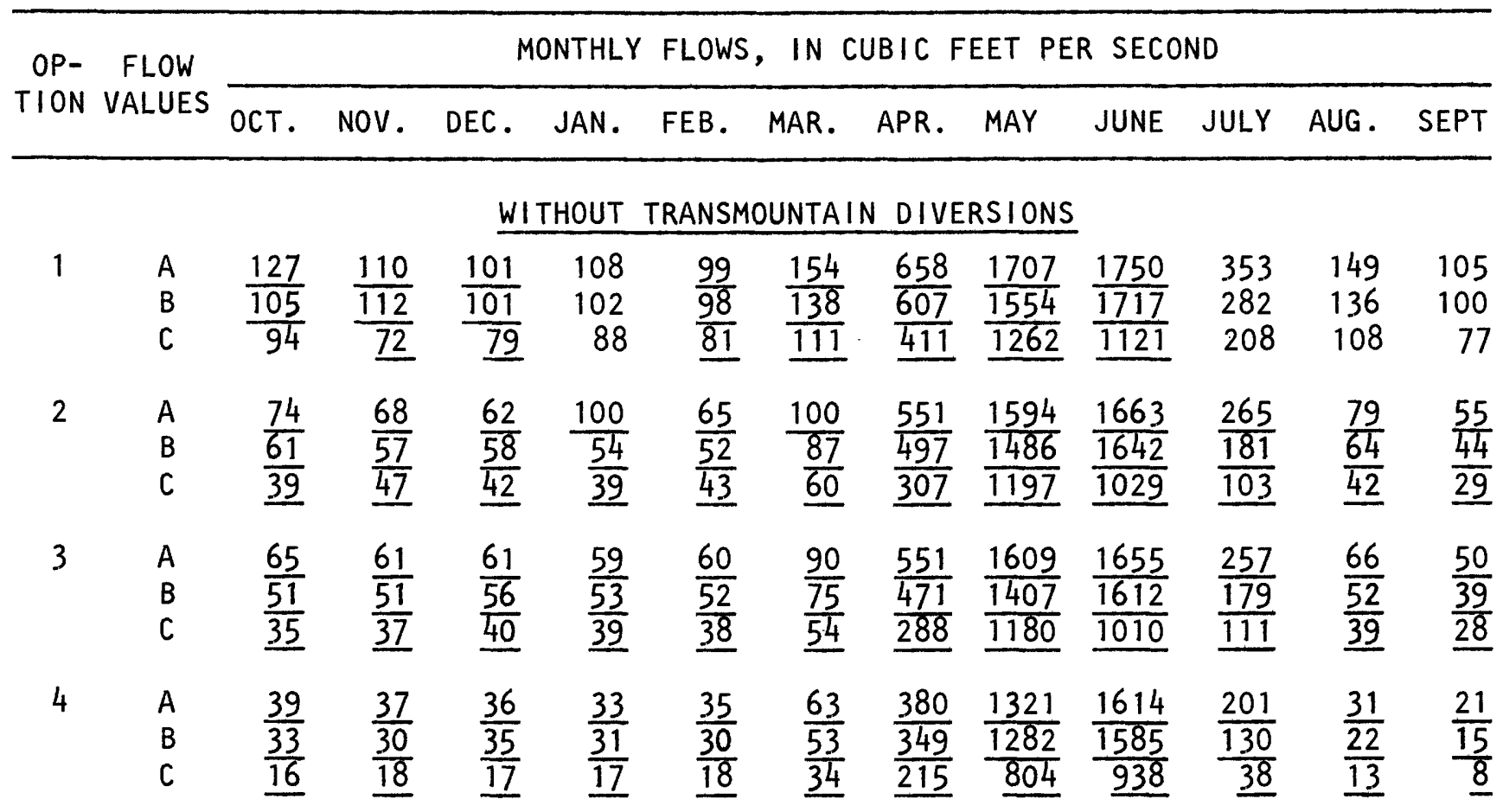
WITH TRANSMOUNTAIN DIVERSIONS

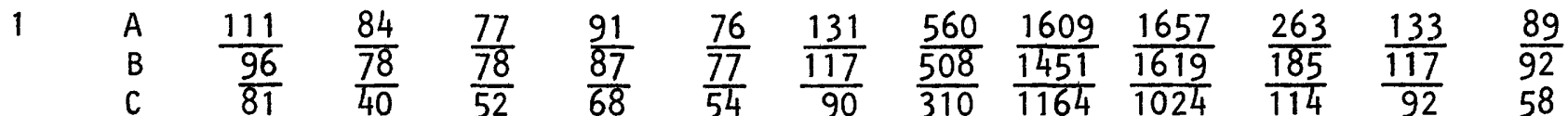

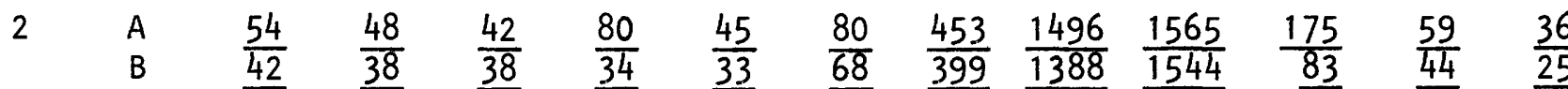

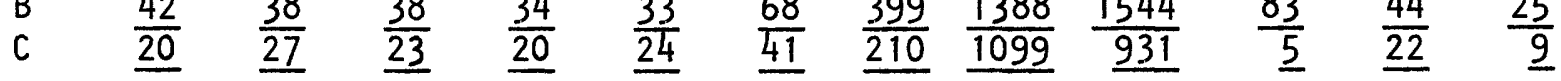

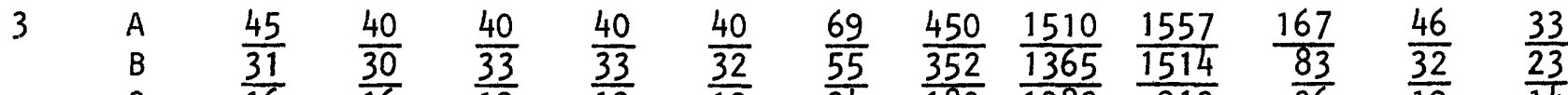

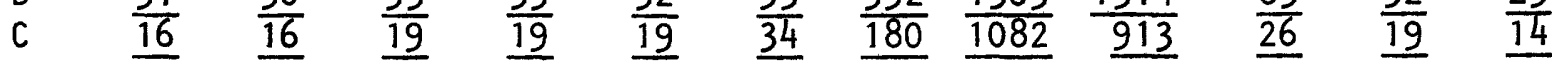

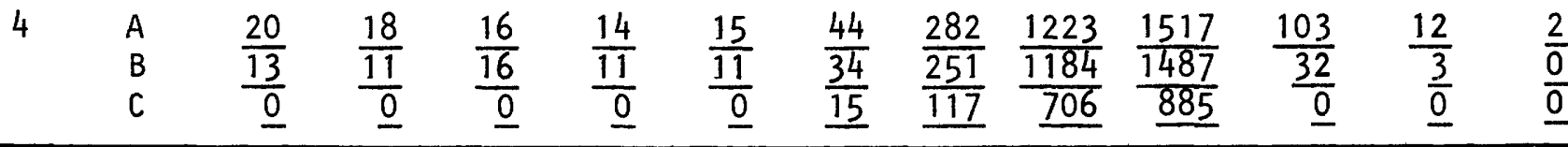


Table 9.--Summary of simulated historical monthly streamflows, control point 39 (Yampa River at Steamboat Springs, Colo.),

with 50 percent of agricultural and no transmountain diversions,

and with 50 percent of both agricultural and transmountain diversions, and including 100 percent of industrial and municipal diversions for all simulations

[FLOW VALUES: $A=$ MEAN; $B=M E D I A N ;$ and $C=80-P E R C E N T$ EXCEEDENCE. Underscored values are less than corresponding table 7 historical conditions]

\begin{tabular}{c} 
MONTHLY FLOWS, IN CUBIC FEET PER SECOND \\
TION VALUES \\
\cline { 2 - 4 } OCT. NOV. DEC. JAN. FEB. MAR. APR. MAY JUNE JULY AUG. SEPT
\end{tabular}

WITHOUT TRANSMOUNTAIN DIVERSIONS

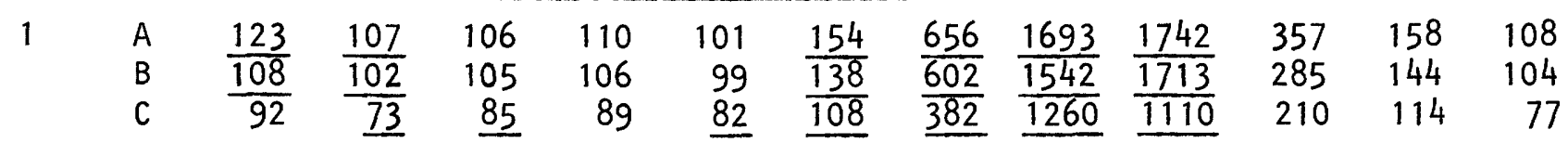

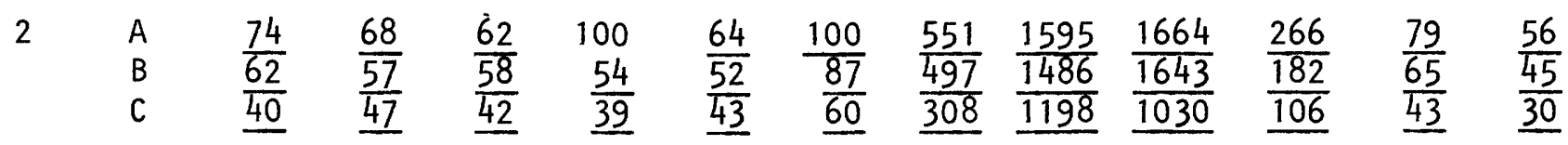

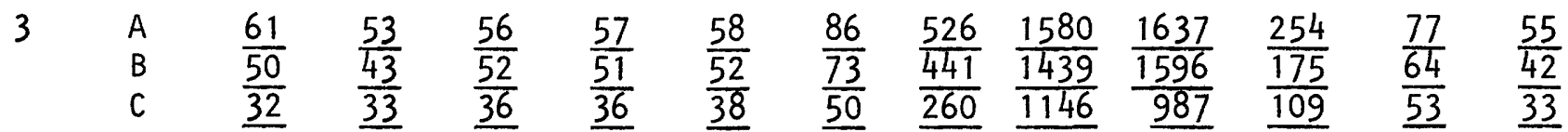

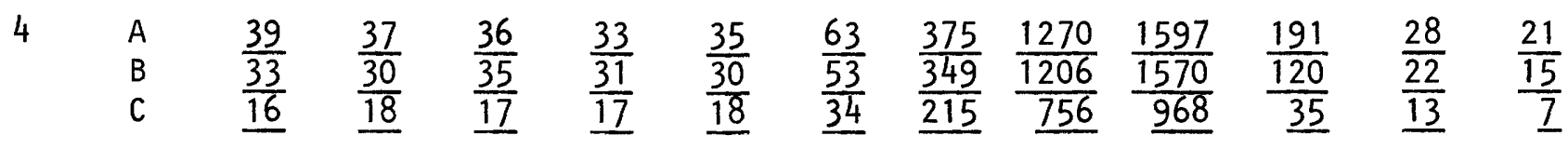

WITH TRANSMOUNTAIN DIVERSIONS

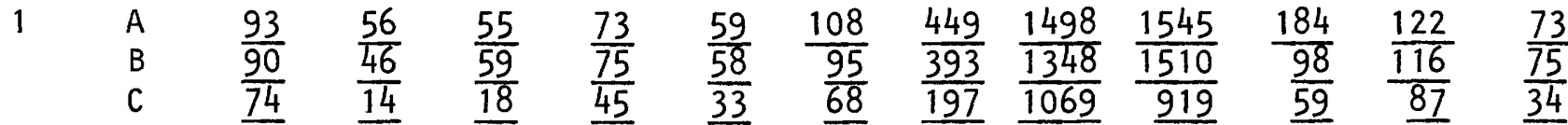

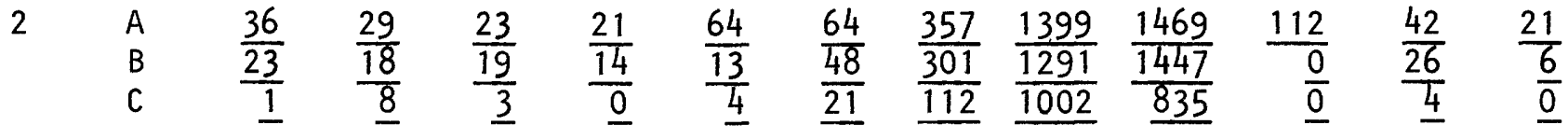

$\begin{array}{lllllllllllll}\text { A } & \frac{25}{10} & \frac{17}{6} & \frac{16}{8} & \frac{19}{11} & \frac{18}{10} & \frac{38}{25} & \frac{314}{184} & \frac{1369}{1217} & \frac{1436}{1396} & \frac{104}{23} & \frac{33}{21} & \frac{21}{8} \\ \text { B } & \frac{1}{1} & \underline{0} & \underline{0} & \underline{1} & \underline{2} & \underline{9} & \underline{913} & \underline{994} & \underline{0} & \underline{0} & \underline{0}\end{array}$

$\begin{array}{llllllllllllll}\text { A } & \underline{0} & \underline{0} & \frac{0}{0} & \frac{0}{0} & \frac{0}{0} & \frac{24}{14} & \frac{180}{153} & \frac{1074}{1011} & \frac{1401}{1375} & \frac{0}{0} & \frac{0}{0} & \frac{0}{0} \\ \text { B } & \underline{0} & \underline{0} & \underline{0} & \underline{0} & \underline{0} & \frac{0}{0} & \frac{2}{20} & \frac{560}{560} & \frac{773}{0} & \underline{0} & \underline{0} & \underline{0}\end{array}$ 
Table 10.--Summary of simulated historical monthly streamflows, control point 39 (Yampa River at Steamboat Springs, Colo.), with 75 percent of agricultural and no transmountain diversions, and with 75 percent of both agricultural and transmountain diversions, and including 100 percent of industrial and municipal diversions for all simulations

[FLOW VALUES: $A=M E A N ; B=M E D I A N$; and $C=80-P E R C E N T$ EXCEEDENCE. Underscored values are less than corresponding table 7 historical conditions]

OCT. NOV. DEC. JAN. FEB. MAR. APR. MAY JUNE JULY AUG. SEPT

\section{WITHOUT TRANSMOUNTAIN DIVERSIONS}

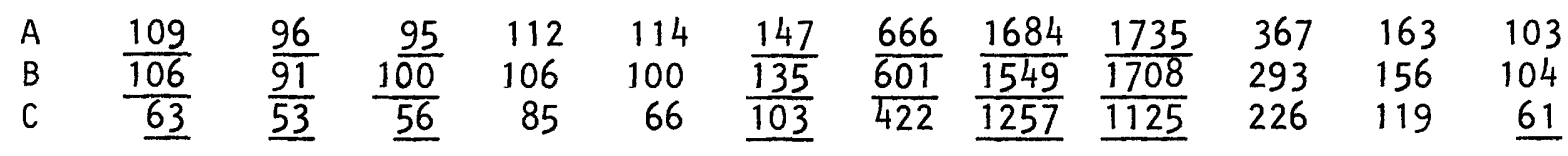

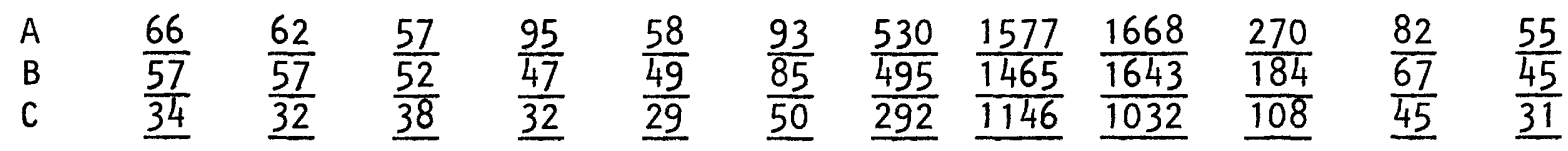

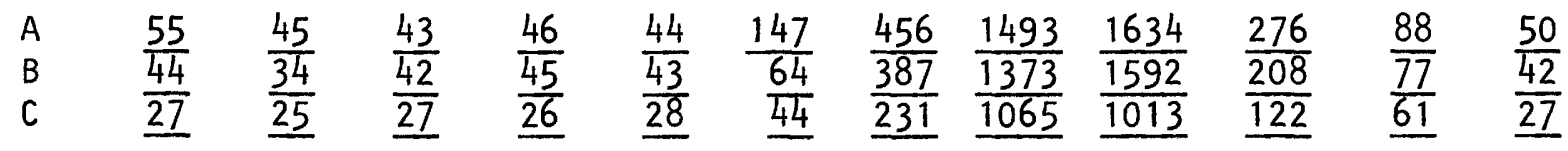

4
$\begin{array}{lll}\text { A } & \frac{39}{33} & \frac{37}{30} \\ \text { B } & \frac{33}{16} & \frac{3}{18}\end{array}$
$\frac{36}{\frac{35}{17}}$
$\frac{\frac{33}{31}}{17} \quad \frac{35}{30}$
$\frac{63}{\frac{53}{34}}$
$\frac{371}{\frac{349}{215}} \quad \frac{1221}{1145} \quad \frac{1581}{712} \quad \frac{1552}{954}$
$\frac{184}{109}$
$\frac{27}{\frac{22}{13}} \quad \frac{21}{15}$

\section{WITH TRANSMOUNTAIN DIVERSIONS}

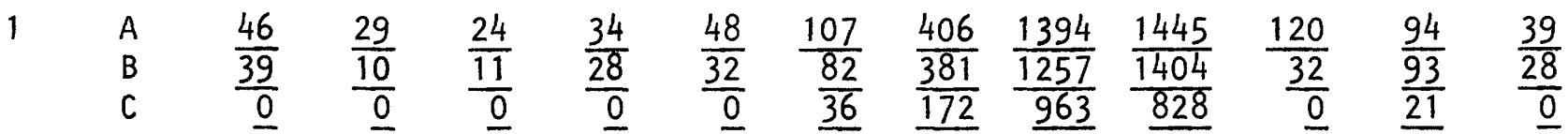

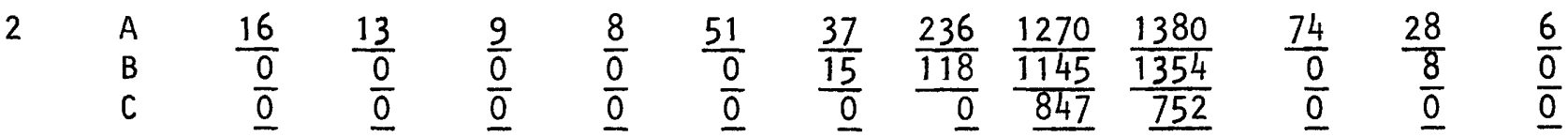

$\begin{array}{llllllllllllll}\text { A } & \frac{16}{0} & \frac{11}{0} & \frac{6}{0} & \frac{7}{0} & \frac{8}{0} & \frac{19}{2} & \frac{199}{64} & \frac{1163}{1038} & \frac{1349}{1309} & \frac{82}{0} & \frac{32}{0} & \frac{15}{0} \\ \text { B } & \underline{0} & \underline{0} & \frac{0}{0} & \underline{0} & \underline{0} & \underline{0} & \underline{0} & \underline{0} 22 & \underline{719} & \underline{0} & \underline{0} & \frac{0}{0}\end{array}$

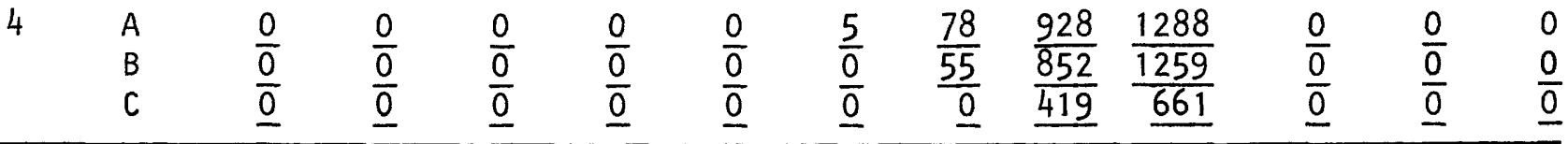


Table 11.--Summary of simulated historical monthly streamflows, control point 39 (Yampa River at Steamboat Springs, Colo.), with 100 percent of agricultural and no transmountain diversions, and with 100 percent of both agricultural and transmountain diversions, and including 100 percent of industrial and municipal diversions for all simulations

[FLOW VALUES: $A=M E A N ; B=M E D I A N ;$ and $C=80-P E R C E N T$ EXCEEDENCE. Underscored values are less than corresponding table 7 historical conditions]

\begin{tabular}{|c|c|c|c|c|c|c|c|c|c|c|c|c|c|}
\hline \multirow{2}{*}{$\begin{array}{l}\text { OP- } \\
\text { TION }\end{array}$} & \multirow{2}{*}{$\begin{array}{l}\text { FLOW } \\
\text { VALUES }\end{array}$} & \multicolumn{12}{|c|}{ MONTHLY FLOWS, IN CUBIC FEET PER SECOND } \\
\hline & & OCT. & NOV. & DEC. & JAN. & FEB. & MAR. & APR. & MAY & JUNE & JULY & AUG. & SEPT \\
\hline & & & & \multicolumn{2}{|c|}{ WITHOUT } & TRANSM & DUNTAI & \multicolumn{2}{|c|}{ DIVERSIONS } & & & & \\
\hline 1 & $\begin{array}{l}A \\
B \\
C\end{array}$ & $\frac{\frac{90}{83}}{\frac{45}{4}}$ & $\frac{\frac{90}{69}}{48}$ & $\frac{\frac{84}{70}}{46}$ & $\begin{array}{r}121 \\
108 \\
49 \\
\end{array}$ & $\begin{array}{r}136 \\
98 \\
49 \\
\end{array}$ & $\frac{\frac{146}{137}}{67}$ & $\begin{array}{l}682 \\
639 \\
423\end{array}$ & $\frac{\frac{1689}{1552}}{1265}$ & $\frac{\frac{1739}{1707}}{1133}$ & $\begin{array}{l}370 \\
306 \\
225\end{array}$ & $\begin{array}{r}145 \\
148 \\
73 \\
\end{array}$ & $\frac{\frac{88}{66}}{\frac{45}{5}}$ \\
\hline 2 & $\begin{array}{l}A \\
B \\
C\end{array}$ & $\frac{\frac{54}{42}}{18}$ & $\frac{\frac{49}{40}}{20}$ & $\frac{\frac{47}{41}}{22}$ & $\frac{\frac{83}{37}}{20}$ & $\frac{\frac{48}{41}}{24}$ & $\frac{\frac{78}{67}}{35}$ & $\frac{\frac{469}{399}}{236}$ & $\frac{\frac{1542}{1414}}{1131}$ & $\frac{\frac{1670}{1643}}{1034}$ & $\frac{\frac{277}{193}}{110}$ & $\frac{\frac{80}{69}}{30}$ & $\frac{\frac{46}{28}}{7}$ \\
\hline 3 & $\begin{array}{l}A \\
B \\
C\end{array}$ & $\frac{\frac{52}{39}}{18}$ & $\frac{\frac{46}{33}}{19}$ & $\frac{\frac{38}{37}}{20}$ & $\frac{\frac{37}{35}}{20}$ & $\frac{\frac{37}{34}}{21}$ & $\frac{107}{\frac{59}{34}}$ & $\frac{\frac{414}{371}}{218}$ & $\frac{\frac{1442}{1371}}{1033}$ & $\frac{\frac{1670}{1614}}{1105}$ & $\frac{\frac{294}{212}}{133}$ & $\frac{\frac{86}{74}}{\frac{20}{20}}$ & $\frac{45}{22}$ \\
\hline 4 & $\begin{array}{l}A \\
B \\
C\end{array}$ & $\frac{\frac{39}{33}}{16}$ & $\frac{\frac{37}{30}}{18}$ & $\frac{\frac{36}{35}}{\frac{17}{7}}$ & $\frac{\frac{33}{31}}{17}$ & $\frac{\frac{35}{30}}{18}$ & $\frac{\frac{63}{53}}{34}$ & $\frac{\frac{370}{349}}{215}$ & $\frac{1175}{\frac{1083}{654}}$ & $\frac{\frac{1565}{1530}}{940}$ & $\frac{\frac{178}{109}}{\underline{35}}$ & $\frac{\frac{25}{22}}{13}$ & $\frac{21}{\frac{15}{7}}$ \\
\hline
\end{tabular}

WITH TRANSMOUNTAIN DIVERSIONS

1

$\begin{array}{lllllllllllll}\text { A } & \underline{7} & \frac{0}{0} & \frac{2}{0} & \frac{23}{0} & \frac{34}{7} & \frac{85}{62} & \frac{349}{252} & \frac{1304}{1182} & \frac{1356}{1312} & \frac{76}{0} & \frac{59}{21} & \frac{5}{0} \\ \text { B } & \underline{0} & \underline{0} & \underline{0} & \underline{0} & \underline{0} & \underline{9} & \underline{124} & \underline{879} & \frac{740}{0} & \underline{0} & \underline{0} & \underline{0}\end{array}$

$\begin{array}{llllllllllllll}\text { A } & \text { A } & \underline{2} & \frac{2}{0} & \underline{3} & \frac{1}{0} & \frac{35}{0} & \frac{22}{0} & \frac{133}{0} & \frac{1142}{1043} & \frac{1283}{1252} & \frac{48}{0} & \frac{18}{0} & \frac{2}{0} \\ \text { B } & \underline{0} & \underline{0} & \underline{0} & \underline{0} & \underline{0} & \underline{0} & \underline{0} & \underline{679} & \underline{643} & \underline{0} & \underline{0} & \underline{0}\end{array}$

$\begin{array}{lllllllllllll}\text { A } & \underline{9} & \underline{6} & \underline{3} & \frac{0}{0} & \underline{0} & \frac{10}{0} & \frac{65}{0} & \frac{1053}{949} & \frac{1307}{1275} & \frac{76}{0} & \frac{37}{0} & \frac{17}{0} \\ \text { B } & \underline{0} & \underline{0} & \underline{0} & \frac{0}{0} & \frac{0}{0} & \underline{0} & \underline{0} & \underline{618} & \underline{708} & \underline{0} & \underline{0} & \underline{0}\end{array}$

$\begin{array}{llllllllllllll}\text { A } & \text { A } & \frac{0}{0} & \frac{0}{0} & \frac{0}{0} & \frac{0}{0} & \frac{0}{0} & \frac{0}{0} & \frac{0}{0} & \frac{784}{691} & \frac{1174}{1139} & \frac{0}{0} & \frac{0}{0} & \frac{0}{0} \\ \text { B } & \underline{0} & \underline{0} & \underline{0} & \underline{0} & \underline{0} & \underline{0} & \underline{0} & \underline{0}\end{array}$ 
Simulated historical streamflow at this control point also showed the potential effects of proposed withdrawals for the Vidler transmountain diversion at the 100-percent water-use allocation level cited in table 7 and the four options cited in tables 8 through 11. Reduced streamflow would occur more frequently as the water-use allocation percentages increase. Zero-flow conditions were found to occur most frequently for reservoir-development option 4 for all levels of water-use allocation. Even the simulated historical conditions with 100 percent of the transmountain diversions indicated zero flow commonly occurring only during July.

Model-simulated historical monthly streamflows for control point 38 (Elk River near Trull, Colo.) are presented in tables 12 through 16. Simulated monthly streamflows for historical conditions without proposed transmountain diversions vary from +1 to -25 percent and have an average absolute variation of 11 percent of the monthly streamflows calculated from streamflow-gaging-station records.

Table 12.--Summary of monthly streamflows, control point 38 (Elk River near Trull, Colo.), for simulated historical conditions, including 100 percent of transmountain diversions, and for historical conditions

[FLOW VALUES: $A=M E A N ; B=M E D I A N ;$ and $C=80-P E R C E N T$ EXCEEDENCE. Underscored values are less than historical conditions without transmountain diversions]

FLOW

MONTHLY FLOWS, IN CUBIC FEET PER SECOND

VALUES OCT. NOV. DEC. JAN. FEB. MAR. APR. MAY JUNE JULY AUG. SEPT SIMULATED HISTORICAL CONDITIONS

$\begin{array}{lllllllllllll}\text { A } & 84 & 76 & 79 & 76 & 90 & 146 & 580 & 1911 & 2082 & 498 & 85 & 57 \\ \text { B } & 83 & 79 & 80 & 75 & 89 & 143 & 561 & 1873 & 2129 & 443 & 82 & 54 \\ \text { C } & 46 & 47 & 62 & 56 & 79 & 118 & 420 & 1476 & 1646 & 137 & 55 & 29\end{array}$

SIMULATED HISTORICAL CONDITIONS WITH 100 PERCENT OF TRANSMOUNTAIN DIVERSIONS

$\begin{array}{lllllllllllll}\text { A } & 84 & 76 & 79 & 76 & 90 & 146 & 580 & 1911 & 2082 & 498 & 85 & 57 \\ \text { B } & 83 & 79 & 80 & 75 & 89 & 143 & 561 & 1873 & 2129 & 443 & 82 & 54 \\ \text { C } & 46 & 47 & 62 & 56 & 79 & 118 & 420 & 1476 & 1646 & 137 & 55 & 29\end{array}$

HISTORICAL STREAMFLOWS CALCULATED FROM GAGING-STATION RECORDS

\begin{tabular}{rrrrrrrrrrrrr} 
A & 109 & 91 & 85 & 79 & 89 & 156 & 633 & 1995 & 2149 & 552 & 113 & 74 \\
B & 110 & 91 & 86 & 78 & 86 & 146 & 580 & 1955 & 2170 & 482 & 100 & 74 \\
C & 57 & 60 & 69 & 55 & 76 & 116 & 434 & 1488 & 1574 & 206 & 62 & 37 \\
\hline
\end{tabular}


Table 13.--Summary of simulated historical monthly streamflows, control point 38 (Elk River near Trull, Colo.),

with 25 percent of agricultural and no transmountain diversions, and with 25 percent of both agricultural and transmountain diversions, and including 100 percent of industrial and municipal diversions for all simulations

[FLOW VALUES: $A=M E A N ; B=M E D I A N$; and $C=80$-PERCENT EXCEEDENCE. Underscored values are less than corresponding table 12 historical conditions]

\begin{tabular}{|c|c|c|c|c|c|c|c|c|c|c|c|c|c|}
\hline \multirow{2}{*}{$\begin{array}{l}\text { OP- } \\
\text { TION }\end{array}$} & \multirow{2}{*}{$\begin{array}{c}\text { FLOW } \\
\text { VALUES }\end{array}$} & \multicolumn{12}{|c|}{ MONTHLY FLOWS, IN CUBIC FEET PER SECOND } \\
\hline & & OCT. & NOV. & DEC. & JAN. & FEB. & MAR. & APR. & MAY & JUNE & JULY & AUG. & SEPT \\
\hline \multicolumn{14}{|c|}{ WITHOUT } \\
\hline 1 & $\begin{array}{l}A \\
B \\
C\end{array}$ & $\begin{array}{l}98 \\
95 \\
61\end{array}$ & $\begin{array}{l}88 \\
90 \\
62\end{array}$ & $\begin{array}{l}88 \\
89 \\
75\end{array}$ & $\begin{array}{l}85 \\
81 \\
65\end{array}$ & $\begin{array}{l}94 \\
93 \\
83\end{array}$ & $\begin{array}{l}147 \\
144 \\
121\end{array}$ & $\frac{\frac{546}{529}}{397}$ & $\frac{\frac{1850}{1800}}{1393}$ & $\frac{\frac{2078}{2123}}{1646}$ & $\begin{array}{l}510 \\
448 \\
159\end{array}$ & $\begin{array}{r}105 \\
103 \\
77\end{array}$ & $\begin{array}{l}75 \\
75 \\
50\end{array}$ \\
\hline 2 & $\begin{array}{l}A \\
B \\
C\end{array}$ & $\begin{array}{r}110 \\
112 \\
84\end{array}$ & $\begin{array}{l}88 \\
91 \\
59\end{array}$ & $\begin{array}{l}87 \\
89 \\
69\end{array}$ & $\begin{array}{l}87 \\
87 \\
64\end{array}$ & $\begin{array}{l}93 \\
93 \\
82\end{array}$ & $\begin{array}{l}146 \\
143 \\
120\end{array}$ & $\frac{\frac{547}{529}}{397}$ & $\frac{\frac{1823}{1742}}{1376}$ & $\frac{\frac{2068}{2098}}{1646}$ & $\begin{array}{l}513 \\
447 \\
172\end{array}$ & $\begin{array}{r}118 \\
116 \\
99\end{array}$ & $\begin{array}{l}87 \\
86 \\
67\end{array}$ \\
\hline 3 & $\begin{array}{l}A \\
B \\
C\end{array}$ & $\begin{array}{l}173 \\
180 \\
146\end{array}$ & $\begin{array}{l}164 \\
167 \\
147\end{array}$ & $\begin{array}{l}163 \\
165 \\
155\end{array}$ & $\begin{array}{l}158 \\
160 \\
148\end{array}$ & $\begin{array}{l}165 \\
167 \\
160\end{array}$ & $\begin{array}{l}200 \\
200 \\
183\end{array}$ & $\frac{\frac{490}{461}}{372}$ & $\frac{\frac{1423}{1326}}{1032}$ & $\frac{\frac{1854}{1831}}{1498}$ & $\begin{array}{l}555 \\
469 \\
285\end{array}$ & $\begin{array}{l}212 \\
215 \\
182\end{array}$ & $\begin{array}{l}172 \\
170 \\
146\end{array}$ \\
\hline 4 & $\begin{array}{l}A \\
B \\
C\end{array}$ & $\frac{\frac{76}{73}}{\frac{41}{4}}$ & $\frac{\frac{68}{71}}{43}$ & $\frac{\frac{77}{79}}{57}$ & $\begin{array}{l}80 \\
80 \\
51 \\
\end{array}$ & $\begin{array}{l}97 \\
97 \\
88\end{array}$ & $\begin{array}{l}153 \\
152 \\
125\end{array}$ & $\begin{array}{l}580 \\
561 \\
425\end{array}$ & $\frac{\frac{1905}{1866}}{1464}$ & $\frac{\frac{2069}{2117}}{1637}$ & $\frac{\frac{485}{430}}{121}$ & $\frac{75}{\frac{72}{47}}$ & $\frac{50}{\frac{48}{24}}$ \\
\hline
\end{tabular}

WITH TRANSMOUNTAIN DIVERSIONS

$\begin{array}{rrrrrrrrrrrrrr}1 & \text { A } & 100 & 88 & 88 & 85 & 94 & 146 & \frac{547}{1842} & \frac{2075}{2105} & 515 & 108 & 76 \\ & \text { B } & 95 & 90 & 89 & 81 & 93 & 144 & \frac{529}{1781} & \frac{2105}{104} & 76 \\ \text { C } & 69 & 62 & 75 & 65 & 83 & 121 & \frac{397}{1394} & \frac{1646}{164} & 79 & 51\end{array}$

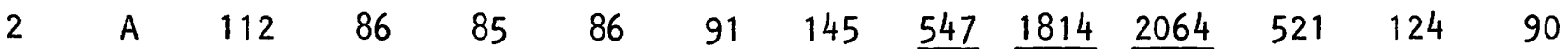

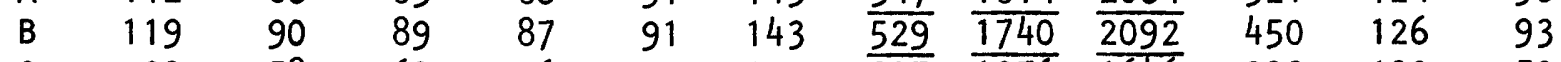

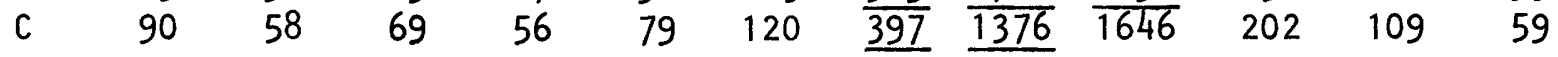

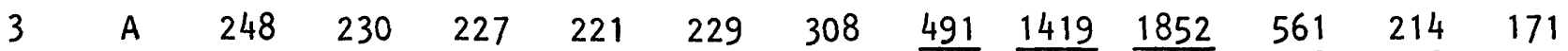

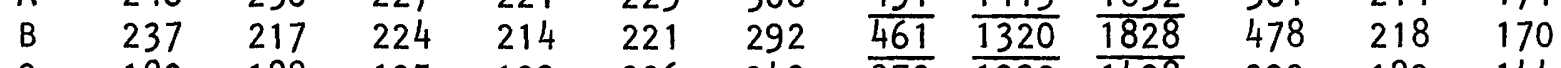

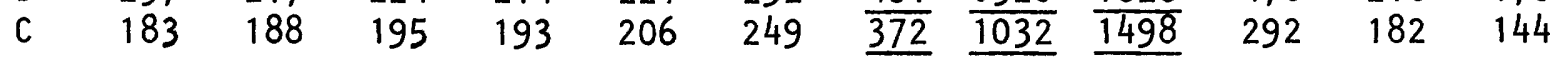

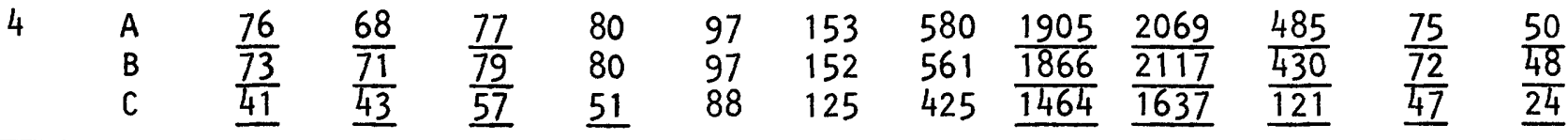


Table 14.--Summary of simulated historical monthly streamflows, control point 38 (Elk River near Trull, Colo.),

with 50 percent of agricultural and no transmountain diversions, and with 50 percent of both agricultural and transmountain diversions, and including 100 percent of industrial and municipal diversions for all simulations

[FLOW VALUES: $A=M E A N ; B=M E D I A N ;$ and $C=80-P E R C E N T$ EXCEEDENCE. Underscored values are less than corresponding table 12 historical conditions]

\begin{tabular}{c} 
MONTHLY FLOWS, IN CUBIC FEET PER SECOND \\
OP- FLOW \\
\cline { 2 - 4 } TION VALUES \\
\cline { 2 - 6 }
\end{tabular}

WITHOUT TRANSMOUNTAIN DIVERSIONS

\begin{tabular}{|c|c|c|c|c|c|c|c|c|c|c|c|c|}
\hline 1 & $\begin{array}{l}A \\
B \\
C\end{array}$ & $\begin{array}{l}98 \\
95 \\
68\end{array}$ & $\begin{array}{l}88 \\
90 \\
62\end{array}$ & $\begin{array}{l}88 \\
89 \\
74\end{array}$ & $\begin{array}{l}85 \\
82 \\
65\end{array}$ & $\begin{array}{l}94 \\
93 \\
83\end{array}$ & $\begin{array}{l}147 \\
143 \\
121\end{array}$ & $\frac{\frac{547}{529}}{397}$ & $\frac{\frac{1838}{1777}}{1398}$ & $\frac{\frac{2074}{2110}}{1646}$ & $\begin{array}{l}517 \\
457 \\
164\end{array}$ & $\begin{array}{r}113 \\
109 \\
83\end{array}$ \\
\hline 2 & $\begin{array}{l}A \\
B \\
C\end{array}$ & $\begin{array}{r}106 \\
115 \\
83\end{array}$ & $\begin{array}{l}86 \\
90 \\
58\end{array}$ & $\begin{array}{l}86 \\
89 \\
69\end{array}$ & $\begin{array}{l}85 \\
87 \\
57\end{array}$ & $\begin{array}{l}92 \\
92 \\
79\end{array}$ & $\begin{array}{l}146 \\
143 \\
120\end{array}$ & $\frac{\frac{547}{529}}{397}$ & $\frac{\frac{1818}{1748}}{1377}$ & $\frac{\frac{2066}{2092}}{1646}$ & $\begin{array}{l}520 \\
458 \\
181\end{array}$ & $\begin{array}{l}127 \\
126 \\
108\end{array}$ \\
\hline 3 & $\begin{array}{l}A \\
B \\
C\end{array}$ & $\begin{array}{l}171 \\
175 \\
147\end{array}$ & $\begin{array}{l}163 \\
166 \\
147\end{array}$ & $\begin{array}{l}163 \\
165 \\
155\end{array}$ & $\begin{array}{l}157 \\
160 \\
148\end{array}$ & $\begin{array}{l}163 \\
167 \\
160\end{array}$ & $\begin{array}{l}198 \\
199 \\
180\end{array}$ & $\frac{\frac{491}{461}}{372}$ & $\frac{\frac{1396}{1308}}{1034}$ & $\frac{\frac{1819}{1842}}{1472}$ & $\begin{array}{l}587 \\
525 \\
310\end{array}$ & $\begin{array}{l}249 \\
255 \\
205\end{array}$ \\
\hline 4 & $\begin{array}{l}A \\
B \\
C\end{array}$ & $\frac{\frac{76}{73}}{\frac{41}{1}}$ & $\frac{\frac{68}{71}}{43}$ & $\frac{77}{\frac{79}{57}}$ & $\begin{array}{l}80 \\
80 \\
51\end{array}$ & $\begin{array}{l}97 \\
97 \\
88\end{array}$ & $\begin{array}{l}153 \\
152 \\
125\end{array}$ & $\begin{array}{l}580 \\
561 \\
425\end{array}$ & $\frac{\frac{1905}{1866}}{1464}$ & $\frac{\frac{2069}{2117}}{1637}$ & $\frac{\frac{485}{430}}{121}$ & $\frac{\frac{75}{72}}{\frac{47}{4}}$ \\
\hline
\end{tabular}

WITH TRANSMOUNTAIN DIVERSIONS

\begin{tabular}{|c|c|c|c|c|c|c|c|c|c|c|c|c|c|}
\hline 1 & $\begin{array}{l}A \\
B \\
C\end{array}$ & $\begin{array}{r}99 \\
100 \\
71\end{array}$ & $\begin{array}{l}85 \\
90 \\
58\end{array}$ & $\begin{array}{l}86 \\
89 \\
69\end{array}$ & $\begin{array}{l}80 \\
81 \\
59\end{array}$ & $\begin{array}{l}95 \\
93 \\
82\end{array}$ & $\begin{array}{l}145 \\
143 \\
120\end{array}$ & $\frac{\frac{547}{529}}{\underline{397}}$ & $\frac{\frac{1832}{1765}}{1384}$ & $\frac{\frac{2068}{2108}}{1646}$ & $\begin{array}{l}535 \\
461 \\
232\end{array}$ & $\begin{array}{r}115 \\
111 \\
93\end{array}$ & $\begin{array}{l}81 \\
81 \\
50\end{array}$ \\
\hline 2 & $\begin{array}{l}A \\
B \\
C\end{array}$ & $\begin{array}{r}106 \\
112 \\
57\end{array}$ & $\begin{array}{l}84 \\
91 \\
50\end{array}$ & $\begin{array}{l}83 \\
82 \\
67\end{array}$ & $\begin{array}{l}80 \\
80 \\
55\end{array}$ & $\begin{array}{l}93 \\
90 \\
79\end{array}$ & $\begin{array}{l}\frac{145}{143} \\
116 \\
\end{array}$ & $\frac{\frac{547}{529}}{397}$ & $\frac{\frac{1808}{1750}}{1376}$ & $\frac{\frac{2062}{2095}}{1646}$ & $\begin{array}{l}537 \\
469 \\
231\end{array}$ & $\begin{array}{l}139 \\
146 \\
119\end{array}$ & \\
\hline 3 & $\begin{array}{l}A \\
B \\
C\end{array}$ & $\begin{array}{l}175 \\
181 \\
150\end{array}$ & $\begin{array}{l}162 \\
166 \\
147\end{array}$ & $\begin{array}{l}161 \\
166 \\
155\end{array}$ & $\begin{array}{l}154 \\
159 \\
145\end{array}$ & $\begin{array}{l}160 \\
167 \\
156\end{array}$ & $\begin{array}{l}194 \\
199 \\
170\end{array}$ & $\frac{\frac{493}{461}}{372}$ & $\frac{\frac{1390}{1308}}{1038}$ & $\frac{\frac{1813}{1834}}{1472}$ & $\begin{array}{l}603 \\
557 \\
317\end{array}$ & $\begin{array}{l}248 \\
260 \\
196\end{array}$ & 14 \\
\hline 4 & $\begin{array}{l}A \\
B \\
C\end{array}$ & $\frac{\frac{76}{73}}{\frac{41}{1}}$ & $\frac{68}{\frac{71}{43}}$ & $\frac{77}{\frac{79}{57}}$ & $\begin{array}{l}80 \\
80 \\
51 \\
\end{array}$ & $\begin{array}{l}97 \\
97 \\
88\end{array}$ & $\begin{array}{l}153 \\
152 \\
125\end{array}$ & $\begin{array}{l}580 \\
561 \\
425\end{array}$ & $\frac{\frac{1905}{1866}}{1464}$ & $\frac{\frac{2069}{2117}}{1637}$ & $\frac{\frac{485}{430}}{121}$ & $\frac{\frac{75}{72}}{\frac{47}{4}}$ & \\
\hline
\end{tabular}


Table 15.--Summary of simulated historical monthly streamflows, control point 38 (Elk River near Trull, Colo.),

with 75 percent of agricultural and no transmountain diversions, and with 75 percent of both agricultural and transmountain diversions, and including 100 percent of industrial and municipal diversions for all simulations

[FLOW VALUES: $A=M E A N ; B=M E D I A N ;$ and $C=80-P E R C E N T$ EXCEEDENCE. Underscored values are less than corresponding table 12 historical conditions]

OP- FLOW

MONTHLY FLOWS, IN CUBIC FEET PER SECOND

TION VALUES

OCT. NOV. DEC. JAN. FEB. MAR. APR. MAY JUNE JULY AUG. SEPT

WITHOUT TRANSMOUNTAIN DIVERSIONS

\begin{tabular}{|c|c|c|c|c|c|c|c|c|c|c|c|}
\hline $\begin{array}{l}A \\
B \\
C\end{array}$ & $\begin{array}{l}93 \\
94 \\
53\end{array}$ & $\begin{array}{l}82 \\
86 \\
55\end{array}$ & $\begin{array}{l}82 \\
84 \\
64\end{array}$ & $\begin{array}{l}81 \\
80 \\
58\end{array}$ & $\begin{array}{l}90 \\
91 \\
77 \\
\end{array}$ & $\frac{\frac{143}{138}}{117}$ & $\frac{\frac{552}{538}}{397}$ & $\frac{\frac{1853}{1843}}{1402}$ & $\frac{\frac{2073}{2121}}{1656}$ & $\begin{array}{l}532 \\
473 \\
184\end{array}$ & $\begin{array}{r}115 \\
118 \\
84\end{array}$ \\
\hline $\begin{array}{l}A \\
B \\
C\end{array}$ & $\begin{array}{r}96 \\
108 \\
52\end{array}$ & $\begin{array}{l}83 \\
86 \\
55\end{array}$ & $\begin{array}{l}83 \\
83 \\
67\end{array}$ & $\begin{array}{l}81 \\
82 \\
55 \\
\end{array}$ & $\begin{array}{l}90 \\
90 \\
77 \\
\end{array}$ & $\begin{array}{l}146 \\
143 \\
117 \\
\end{array}$ & $\frac{\frac{548}{530}}{397}$ & $\frac{\frac{1842}{1842}}{1396}$ & $\frac{\frac{2067}{2104}}{1643}$ & $\begin{array}{l}534 \\
476 \\
198\end{array}$ & $\begin{array}{r}128 \\
139 \\
83\end{array}$ \\
\hline $\begin{array}{l}\text { A } \\
B \\
C\end{array}$ & $\begin{array}{r}143 \\
175 \\
53\end{array}$ & $\begin{array}{r}126 \\
150 \\
51\end{array}$ & $\begin{array}{r}117 \\
149 \\
48 \\
\end{array}$ & $\begin{array}{r}110 \\
121 \\
41 \\
\end{array}$ & $\begin{array}{r}115 \\
102 \\
53 \\
\end{array}$ & $\begin{array}{r}185 \\
169 \\
88 \\
\end{array}$ & $\frac{\frac{452}{451}}{332}$ & $\frac{\frac{1491}{1527}}{1066}$ & $\frac{\frac{1841}{1865}}{1488}$ & $\begin{array}{l}682 \\
648 \\
408\end{array}$ & $\begin{array}{l}299 \\
308 \\
244\end{array}$ \\
\hline $\begin{array}{l}A \\
B \\
C\end{array}$ & $\frac{\frac{76}{73}}{41}$ & $\frac{\frac{68}{71}}{43}$ & $\frac{\frac{77}{79}}{57}$ & $\begin{array}{l}80 \\
80 \\
51\end{array}$ & $\begin{array}{l}97 \\
97 \\
88\end{array}$ & $\begin{array}{l}153 \\
152 \\
125\end{array}$ & $\begin{array}{l}580 \\
561 \\
425\end{array}$ & $\frac{\frac{1905}{1866}}{1464}$ & $\frac{\frac{2069}{2117}}{1637}$ & $\frac{\frac{485}{430}}{121}$ & $\frac{75}{\frac{72}{47}}$ \\
\hline
\end{tabular}

WITH TRANSMOUNTAIN DIVERSIONS

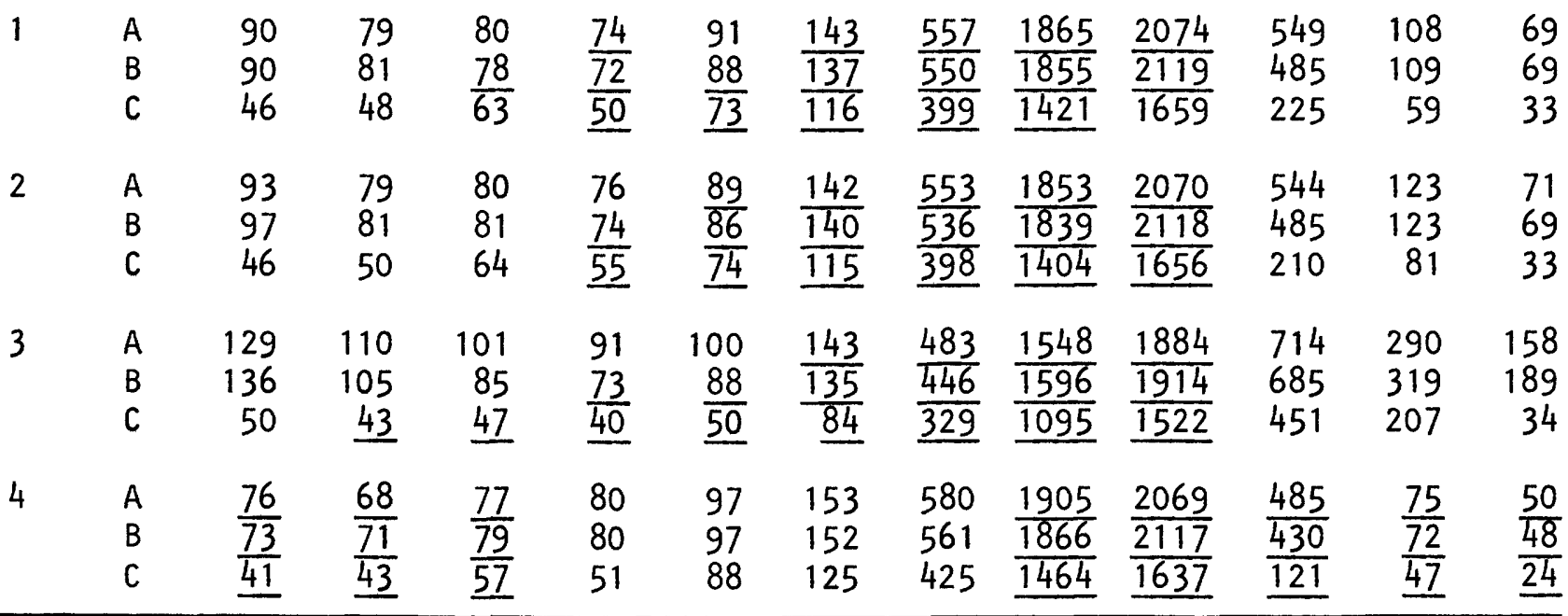


Table 16.--Summary of simulated historical monthly streamflows, control point 38 (Elk River near Tmull, Colo.),

with 100 percent of agricultural and no transmountain diversions, and with 100 percent of both agricultural and transmountain diversions, and including 100 percent of industrial and municipal diversions for all simulations

[FLOW VALUES: $A=M E A N ; B=M E D I A N ;$ and $C=80-P E R C E N T$ EXCEEDENCE. Underscored values are less than corresponding table 12 historical conditions]

\begin{tabular}{|c|c|c|c|c|c|c|c|c|c|c|c|c|c|}
\hline \multirow{2}{*}{$\begin{array}{c}\text { OP- } \\
\text { TION }\end{array}$} & \multirow{2}{*}{$\begin{array}{l}\text { FLOW } \\
\text { VALUES }\end{array}$} & \multicolumn{12}{|c|}{ MONTHLY FLOWS, IN CUBIC FEET PER SECOND } \\
\hline & & OCT. & NOV. & DEC. & JAN. & FEB. & MAR. & APR. & MAY & JUNE & JULY & AUG. & SEPT \\
\hline \multicolumn{14}{|c|}{ WITHOUT TRANSMOUNTAIN DIVERSIONS } \\
\hline 1 & $\begin{array}{l}A \\
B \\
C\end{array}$ & $\begin{array}{l}86 \\
81 \\
46\end{array}$ & $\begin{array}{l}77 \\
79 \\
54\end{array}$ & $\begin{array}{l}78 \\
75 \\
62\end{array}$ & $\begin{array}{l}79 \\
74 \\
50\end{array}$ & $\begin{array}{l}90 \\
90 \\
76\end{array}$ & $\frac{\frac{141}{139}}{111}$ & $\frac{\frac{558}{539}}{\underline{402}}$ & $\frac{\frac{1878}{1858}}{1416}$ & $\frac{\frac{2081}{2125}}{1667}$ & $\begin{array}{l}538 \\
481 \\
185\end{array}$ & $\begin{array}{r}106 \\
109 \\
59\end{array}$ & $\begin{array}{l}65 \\
67 \\
31\end{array}$ \\
\hline 2 & $\begin{array}{l}A \\
B \\
C\end{array}$ & $\begin{array}{l}87 \\
84 \\
46\end{array}$ & $\begin{array}{l}77 \\
\frac{76}{54}\end{array}$ & $\begin{array}{l}79 \\
81 \\
62\end{array}$ & $\begin{array}{l}77 \\
74 \\
50 \\
\end{array}$ & $\begin{array}{l}\frac{89}{91} \\
76 \\
\end{array}$ & $\frac{\frac{143}{140}}{112}$ & $\begin{array}{l}\frac{554}{540} \\
\underline{397}\end{array}$ & $\frac{\frac{1873}{1862}}{1416}$ & $\frac{\frac{2075}{2120}}{1662}$ & $\begin{array}{l}540 \\
482 \\
186\end{array}$ & $\begin{array}{r}114 \\
113 \\
66\end{array}$ & $\begin{array}{l}67 \\
68 \\
32\end{array}$ \\
\hline 3 & $\begin{array}{l}A \\
B \\
C\end{array}$ & $\begin{array}{l}92 \\
68 \\
31\end{array}$ & $\begin{array}{l}80 \\
58 \\
37 \\
\end{array}$ & $\frac{\frac{73}{53}}{43}$ & $\frac{\frac{71}{52}}{33}$ & $\frac{\frac{77}{54}}{49}$ & $\frac{138}{\frac{96}{75}}$ & $\frac{\frac{451}{405}}{306}$ & $\frac{\frac{1650}{1614}}{1253}$ & $\frac{\frac{1956}{1973}}{1573}$ & $\begin{array}{l}772 \\
735 \\
518\end{array}$ & $\begin{array}{l}284 \\
337 \\
130\end{array}$ & $\begin{array}{r}115 \\
54 \\
22\end{array}$ \\
\hline 4 & $\begin{array}{l}A \\
B \\
C\end{array}$ & $\frac{76}{\frac{73}{41}}$ & $\frac{\frac{68}{71}}{43}$ & $\frac{77}{\frac{79}{57}}$ & $\begin{array}{l}80 \\
80 \\
51 \\
\end{array}$ & $\begin{array}{l}97 \\
97 \\
88\end{array}$ & $\begin{array}{l}153 \\
152 \\
125\end{array}$ & $\begin{array}{l}580 \\
561 \\
425\end{array}$ & $\frac{\frac{1905}{1866}}{1464}$ & $\frac{\frac{2069}{2117}}{1637}$ & $\frac{\frac{485}{430}}{121}$ & $\frac{\frac{75}{72}}{\frac{47}{4}}$ & $\frac{50}{\frac{48}{24}}$ \\
\hline \multicolumn{14}{|c|}{ WITH TRANSMOUNTAIN DIVERSIONS } \\
\hline 1 & $\begin{array}{l}A \\
B \\
C\end{array}$ & $\begin{array}{l}84 \\
81 \\
46\end{array}$ & $\frac{75}{\frac{75}{47}}$ & $\frac{\frac{77}{75}}{62}$ & $\frac{\frac{73}{71}}{50}$ & $\begin{array}{l}90 \\
81 \\
69 \\
\end{array}$ & $\frac{\frac{140}{131}}{106}$ & $\frac{566}{\frac{559}{409}}$ & $\frac{\frac{1892}{1867}}{1459}$ & $\begin{array}{l}2085 \\
2132 \\
1670\end{array}$ & $\begin{array}{l}538 \\
480 \\
188\end{array}$ & $\begin{array}{l}99 \\
84 \\
56\end{array}$ & $\begin{array}{l}59 \\
56 \\
29\end{array}$ \\
\hline 2 & $\begin{array}{l}A \\
B \\
C\end{array}$ & $\begin{array}{l}85 \\
89 \\
46\end{array}$ & $\begin{array}{l}77 \\
\frac{76}{47}\end{array}$ & $\frac{\frac{77}{75}}{62}$ & $\frac{\frac{73}{71}}{50}$ & $\begin{array}{l}91 \\
85 \\
72 \\
\end{array}$ & $\frac{\frac{144}{142}}{116}$ & $\frac{\frac{557}{553}}{397}$ & $\frac{\frac{1884}{1864}}{1424}$ & $\frac{\frac{2079}{2124}}{1668}$ & $\begin{array}{l}543 \\
491 \\
195\end{array}$ & $\begin{array}{r}104 \\
95 \\
56\end{array}$ & $\begin{array}{l}63 \\
66 \\
29\end{array}$ \\
\hline 3 & $\begin{array}{l}A \\
B \\
C\end{array}$ & $\frac{77}{\frac{74}{35}}$ & $\frac{\frac{70}{53}}{37}$ & $\frac{\frac{66}{51}}{\frac{43}{4}}$ & $\frac{58}{47}$ & $\frac{\frac{70}{53}}{\frac{48}{4}}$ & $\frac{115}{\frac{91}{72}}$ & $\begin{array}{l}423 \\
385 \\
291\end{array}$ & $\begin{array}{l}1769 \\
1755 \\
1403\end{array}$ & $\begin{array}{l}2003 \\
2010 \\
1605\end{array}$ & $\begin{array}{l}789 \\
793 \\
515\end{array}$ & $\begin{array}{r}222 \\
156 \\
40\end{array}$ & $\begin{array}{r}100 \\
49 \\
23\end{array}$ \\
\hline 4 & $\begin{array}{l}A \\
B \\
C\end{array}$ & $\frac{\frac{76}{73}}{\frac{41}{4}}$ & $\frac{\frac{68}{71}}{43}$ & $\frac{\frac{77}{79}}{57}$ & $\begin{array}{l}80 \\
80 \\
51 \\
\end{array}$ & $\begin{array}{l}97 \\
97 \\
88\end{array}$ & $\begin{array}{l}153 \\
152 \\
125\end{array}$ & $\begin{array}{l}580 \\
561 \\
425\end{array}$ & $\frac{\frac{1905}{1866}}{1464}$ & $\frac{\frac{2069}{2117}}{1637}$ & $\frac{\frac{485}{430}}{121}$ & $\frac{\frac{75}{72}}{47}$ & $\frac{\frac{50}{48}}{24}$ \\
\hline
\end{tabular}


The effects of agricultural and transmountain diversions were reduced at this site because no proposed diversions were considered for the proposed Hinman Park or Grouse Mountain Reservoirs. In reservoir-development options 1 and 2, the 50year flow statistics (tables 12 through 16) have responded to an increased demand from the downstream Yampa main-stem reservoirs, principally Juniper and Cross Mountain Reservoirs, by a slight reduction in the peak flow months (April to June). Reservoir-development options 3 and 4 included the proposed Hinman Park and Grouse Mountain Reservoirs upstream and tended to even out the monthly flow cycle. In reservoir-development option 3, more water had been released from Hinman Park and Grouse Mountain Reservoirs to meet the demand from the Juniper and Cross Mountain Reservoirs during the irrigation season. Reservoir-development option 4 includes the Hinman Park and Grouse Mountain Reservoirs, but the downstream demand from Juniper and Cross Mountain Reservoirs is not included; consequently the flow did not vary with increased water-use allocations. Increasing the water-use allocation percentages generally could increase the number of months that the flow statistics are less than the historical conditions (underscored statistics, tables 12 through 16), especially during the irrigation season (April to October). The upstream reservoir could cause a reduction in peak-flow months and a flow increase during the low-flow, high water-use irrigation months.

The transmountain diversions have little or no effect in reservoir-development options 1, 2, and 4. Only in reservoir-development option 3, where the large downstream reservoirs were requiring water to replace the Vidler transmountain diversion water taken from the Steamboat Springs location, can any real effect on the flow statistics be noticed for the Elk River near Trull, Colo.

Model-simulated historical monthly streamflows for control point 34 (Trout Creek at mouth) are presented in tables 17 through 21; the general location of this site is shown in figure 1. The effects of agricultural and transmountain diversions would be negligible in many instances at this control point. The effects of the proposed diversions for the Oak Creek Water and Power Project are indicated by the data for reservoir-development options 2, 3, and 4. The Oak Creek power complex includes only industrial diversions; therefore, very little change in monthly flow statistics can be noticed with changes in water-use allocation (tables 18 through 21). Reservoir-development option-4 monthly streamflows were slightly reduced because the Juniper and Cross Mountain Reservoirs were not in operation and did not require upstream inflow to fulfill diversion requirements. 
Table 17.--Summary of simulated historical monthly streamflows, control point 34 (Trout Creek at mouth), for

historical conditions and with 100 percent of transmountain diversions

[FLOW VALUES: $A=M E A N ; B=M E D I A N ;$ and $C=80-P E R C E N T$ EXCEEDENCE]

FLOW

MONTHLY FLOWS, IN CUBIC FEET PER SECOND

VALUES OCT. NOV. DEC. JAN. FEB. MAR. APR. MAY JUNE JULY AUG. SEPT

SIMULATED HISTORICAL CONDITIONS WITHOUT TRANSMOUNTAIN DIVERSIONS

$\begin{array}{rrrrrrrrrrrrr}\text { A } & 21 & 25 & 24 & 23 & 27 & 41 & 158 & 297 & 103 & 22 & 14 & 14 \\ \text { B } & 17 & 23 & 23 & 23 & 29 & 40 & 128 & 243 & 88 & 17 & 11 & 11 \\ \text { C } & 11 & 17 & 17 & 17 & 23 & 29 & 91 & 166 & 40 & 11 & 6 & 11\end{array}$

SIMULATED HISTORICAL CONDITIONS WITH 100 PERCENT OF TRANSMOUNTAIN DIVERSIONS

$\begin{array}{rrrrrrrrrrrrr}\text { A } & 21 & 25 & 24 & 23 & 27 & 41 & 158 & 297 & 103 & 22 & 14 & 14 \\ \text { B } & 17 & 23 & 23 & 23 & 29 & 40 & 128 & 243 & 88 & 17 & 11 & 11 \\ \text { C } & 11 & 17 & 17 & 17 & 23 & 29 & 91 & 166 & 40 & 11 & 6 & 11\end{array}$


Table 18.--Summary of simulated historical monthly streamflows, control point 34 (Trout Creek at mouth),

with 25 percent of agricultural and no transmountain diversions, and with 25 percent of both agricultural and transmountain diversions, and including 100 percent of industrial and municipal diversions for all simulations

[FLOW VALUES: $A=M E A N ; B=M E D I A N ;$ and $C=80-P E R C E N T$ EXCEEDANCE. Underscored values are less than corresponding table 17 simulated historical conditions]

\begin{tabular}{ccc}
\hline MONTHLY FLOWS, IN CUBIC FEET PER SECOND \\
TION VALUES \\
\cline { 2 - 3 }
\end{tabular}

WITHOUT TRANSMOUNTAIN DIVERSIONS

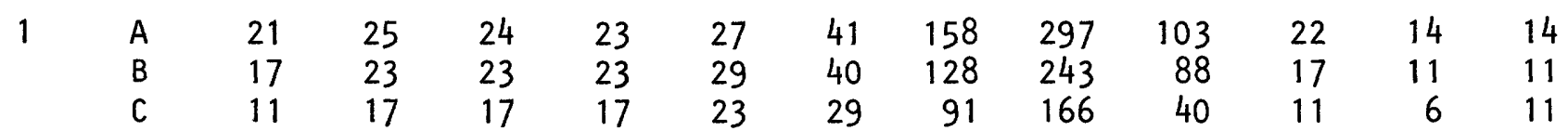

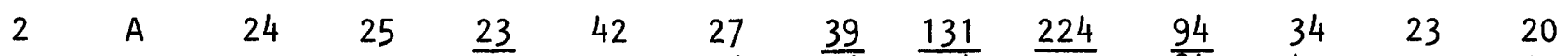

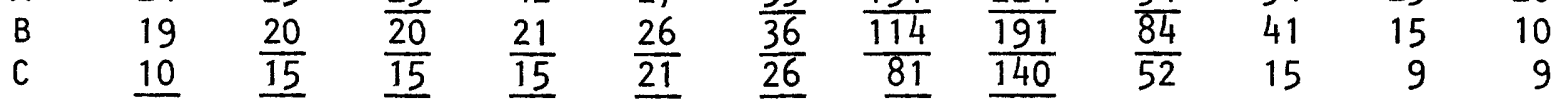

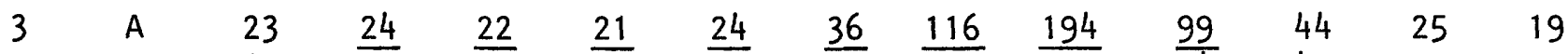

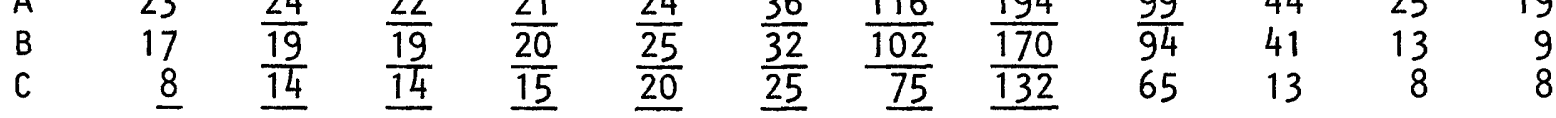

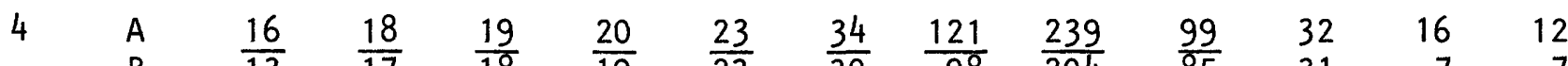

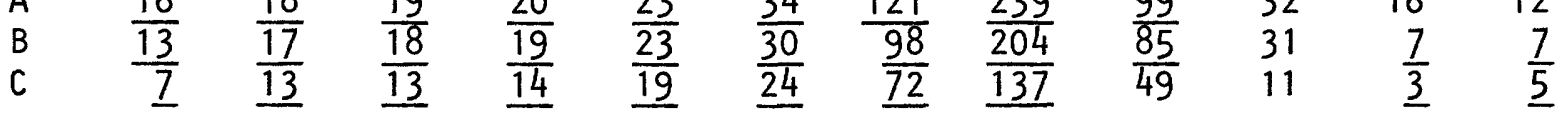

WITH TRANSMOUNTAIN DIVERSIONS

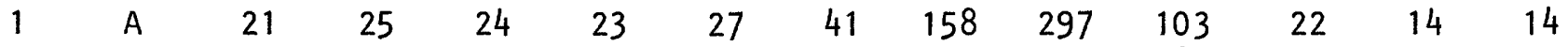

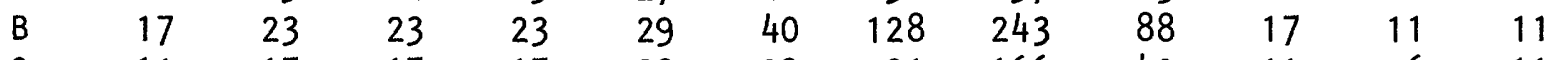

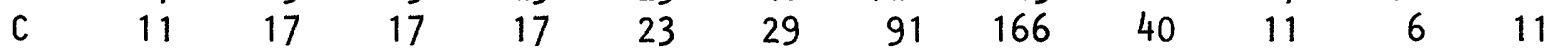

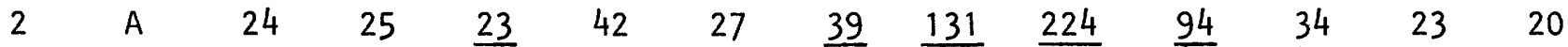

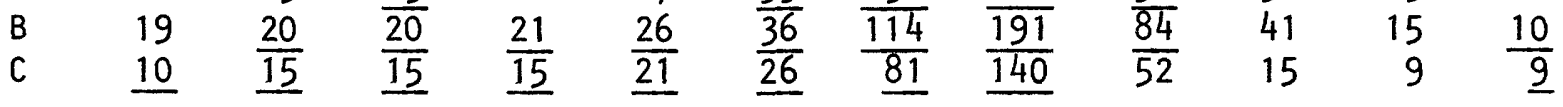

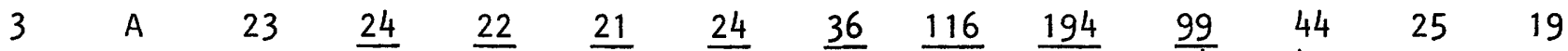

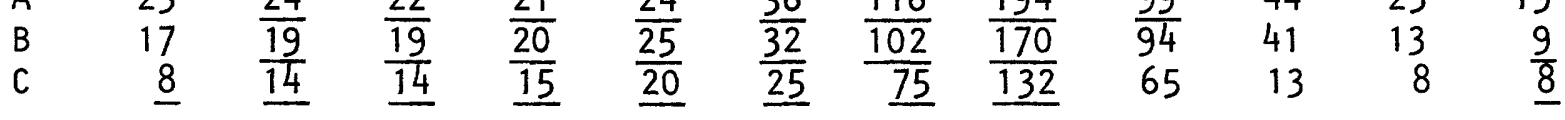

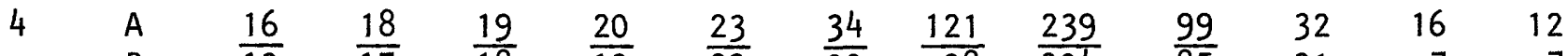

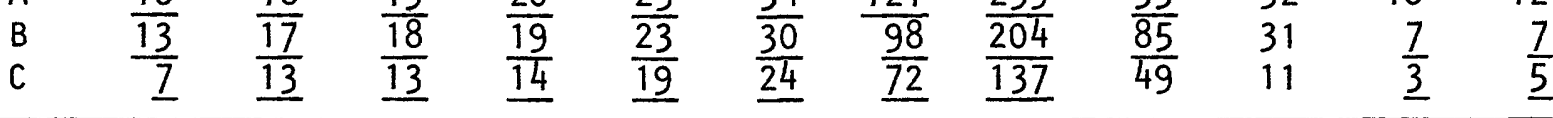


Table 19.--Summary of simulated historical monthly streamflows, control point 34 (Trout Creek at mouth),

with 50 percent of agricultural and no transmountain diversions, and with 50 percent of both agricultural and transmountain diversions, and including 100 percent of industrial and municipal diversions for all simulations

[FLOW VALUES: $A=M E A N ; B=M E D I A N ;$ and $C=80-P E R C E N T$ EXCEEDENCE. Underscored values are less than corresponding table 17 simulated historical conditions]

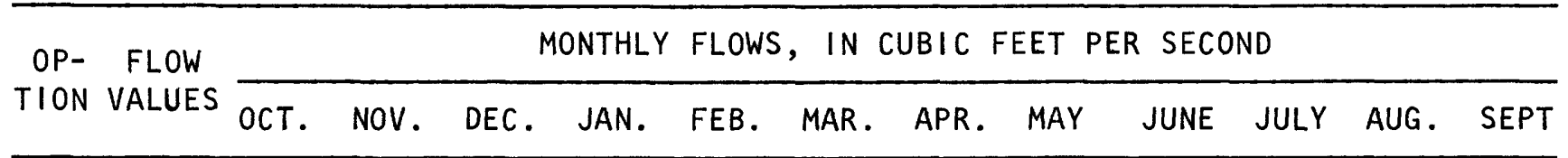

WITHOUT TRANSMOUNTAIN DIVERSIONS

\begin{tabular}{|c|c|c|c|c|c|c|c|c|c|c|c|c|}
\hline 1 & $\begin{array}{l}A \\
B \\
C\end{array}$ & $\begin{array}{l}21 \\
17 \\
11\end{array}$ & $\begin{array}{l}25 \\
23 \\
17\end{array}$ & $\begin{array}{l}24 \\
23 \\
17\end{array}$ & $\begin{array}{l}23 \\
23 \\
17\end{array}$ & $\begin{array}{l}27 \\
29 \\
23\end{array}$ & $\begin{array}{l}41 \\
40 \\
29\end{array}$ & $\begin{array}{r}158 \\
128 \\
91\end{array}$ & $\begin{array}{l}297 \\
243 \\
166\end{array}$ & $\begin{array}{r}103 \\
88 \\
40\end{array}$ & $\begin{array}{l}22 \\
17 \\
11\end{array}$ & $\begin{array}{r}14 \\
11 \\
6\end{array}$ \\
\hline 2 & $\begin{array}{l}A \\
B \\
C\end{array}$ & $\begin{array}{l}24 \\
19 \\
10 \\
\end{array}$ & $\begin{array}{l}25 \\
20 \\
15 \\
\end{array}$ & $\frac{23}{\frac{23}{15}}$ & $\begin{array}{l}42 \\
21 \\
15 \\
\end{array}$ & $\begin{array}{l}27 \\
\frac{26}{21} \\
\end{array}$ & $\frac{\frac{39}{36}}{26}$ & $\frac{\frac{131}{114}}{81}$ & $\frac{\frac{224}{191}}{140}$ & $\frac{\frac{94}{84}}{52}$ & $\begin{array}{l}34 \\
41 \\
15\end{array}$ & $\begin{array}{r}23 \\
15 \\
9\end{array}$ \\
\hline 3 & $\begin{array}{l}A \\
B \\
C\end{array}$ & $\begin{array}{r}23 \\
18 \\
8 \\
\end{array}$ & $\frac{\frac{23}{19}}{14}$ & $\frac{\frac{22}{19}}{14}$ & $\frac{\frac{21}{19}}{14}$ & $\frac{\frac{24}{24}}{19}$ & $\frac{\frac{36}{32}}{\frac{24}{24}}$ & $\frac{\frac{120}{107}}{75}$ & $\frac{\frac{203}{176}}{131}$ & $\begin{array}{l}\frac{98}{88} \\
55\end{array}$ & $\begin{array}{l}34 \\
39 \\
13\end{array}$ & $\begin{array}{r}21 \\
13 \\
8\end{array}$ \\
\hline 4 & $\begin{array}{l}A \\
B \\
C\end{array}$ & $\frac{\frac{15}{13}}{7}$ & $\frac{19}{\frac{18}{13}}$ & $\frac{\frac{20}{18}}{13}$ & $\frac{\frac{21}{20}}{15}$ & $\frac{24}{\frac{24}{20}}$ & $\frac{35}{\frac{32}{26}}$ & $\frac{126}{104}$ & $\frac{\frac{251}{218}}{145}$ & $\frac{98}{\frac{94}{35}}$ & $\frac{\frac{20}{14}}{6}$ & $\frac{9}{6}$ \\
\hline 1 & $\begin{array}{l}A \\
B \\
C\end{array}$ & $\begin{array}{l}21 \\
17 \\
11\end{array}$ & $\begin{array}{l}25 \\
23 \\
17\end{array}$ & $\begin{array}{l}24 \\
23 \\
17\end{array}$ & $\begin{array}{l}23 \\
23 \\
17\end{array}$ & $\begin{array}{l}27 \\
29 \\
23\end{array}$ & $\begin{array}{l}41 \\
40 \\
29\end{array}$ & $\begin{array}{r}158 \\
128 \\
91\end{array}$ & $\begin{array}{l}297 \\
243 \\
166\end{array}$ & $\begin{array}{r}103 \\
88 \\
40\end{array}$ & $\begin{array}{l}22 \\
17 \\
11\end{array}$ & $\begin{array}{r}14 \\
11 \\
6\end{array}$ \\
\hline 2 & $\begin{array}{l}A \\
B \\
C\end{array}$ & $\begin{array}{l}24 \\
19 \\
10 \\
\end{array}$ & $\begin{array}{l}25 \\
20 \\
15 \\
\end{array}$ & $\frac{\frac{23}{20}}{15}$ & $\begin{array}{l}22 \\
21 \\
15 \\
\end{array}$ & $\begin{array}{l}47 \\
26 \\
21 \\
\end{array}$ & $\frac{\frac{39}{36}}{26}$ & $\frac{\frac{131}{114}}{81}$ & $\frac{\frac{224}{191}}{140}$ & $\frac{\frac{94}{84}}{52}$ & $\begin{array}{l}34 \\
41 \\
15\end{array}$ & $\begin{array}{r}23 \\
15 \\
9\end{array}$ \\
\hline 3 & $\begin{array}{l}A \\
B \\
C\end{array}$ & $\begin{array}{r}23 \\
18 \\
8 \\
\end{array}$ & $\frac{\frac{23}{19}}{14}$ & $\frac{\frac{22}{19}}{14}$ & $\frac{\frac{21}{19}}{14}$ & $\frac{\frac{24}{24}}{19}$ & $\frac{\frac{36}{32}}{\frac{34}{24}}$ & $\frac{\frac{120}{107}}{75}$ & $\frac{\frac{202}{176}}{131}$ & $\begin{array}{l}\frac{98}{88} \\
55\end{array}$ & $\begin{array}{l}34 \\
39 \\
13\end{array}$ & $\begin{array}{r}21 \\
13 \\
8\end{array}$ \\
\hline 4 & $\begin{array}{l}A \\
B \\
C\end{array}$ & $\frac{\frac{15}{13}}{7}$ & $\frac{\frac{19}{18}}{13}$ & $\frac{\frac{20}{18}}{13}$ & $\frac{\frac{21}{20}}{15}$ & $\frac{\frac{24}{24}}{20}$ & $\frac{\frac{35}{32}}{\frac{26}{2}}$ & $\frac{126}{104}$ & $\frac{\frac{251}{218}}{145}$ & $\frac{\frac{98}{84}}{35}$ & $\frac{\frac{20}{14}}{6}$ & $\frac{9}{\frac{6}{3}}$ \\
\hline
\end{tabular}


Table 20.--Summary of simulated historical monthly streamflows, control point 34 (Trout Creek at mouth),

with 75 percent of agricultural and no transmountain diversions, and with 75 percent of both agricultural and transmountain diversions, and including 100 percent of industrial and municipal diversions for all simulations

[FLOW VALUES: $A=$ MEAN; $B=$ MEDIAN; and $C=80-P E R C E N T$ EXCEEDENCE. Underscored values are less than corresponding table 17 simulated historical conditions]

OP- FLOW

MONTHLY FLOWS, IN CUBIC FEET PER SECOND

TION VALUES OCT. NOV. DEC. JAN. FEB. MAR. APR. MAY JUNE JULY AUG. SEPT

WITHOUT TRANSMOUNTAIN DIVERSIONS

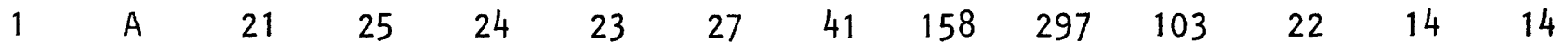

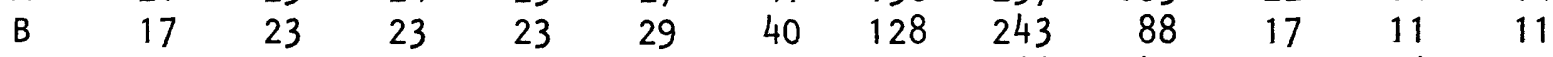

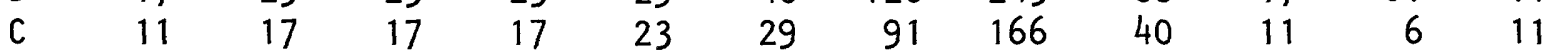

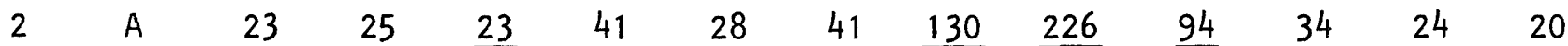

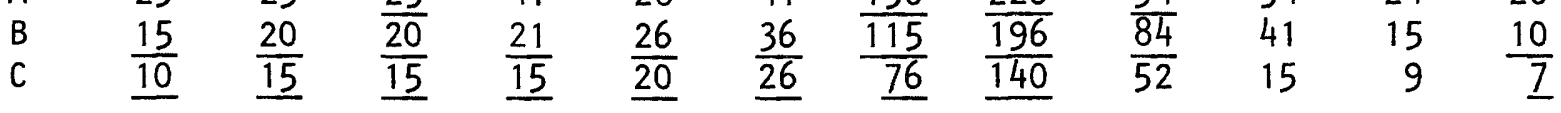

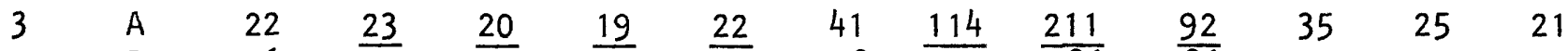

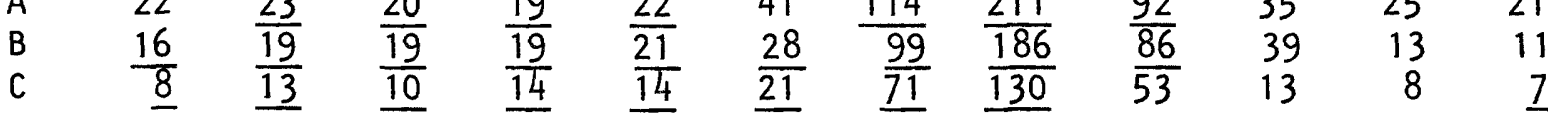

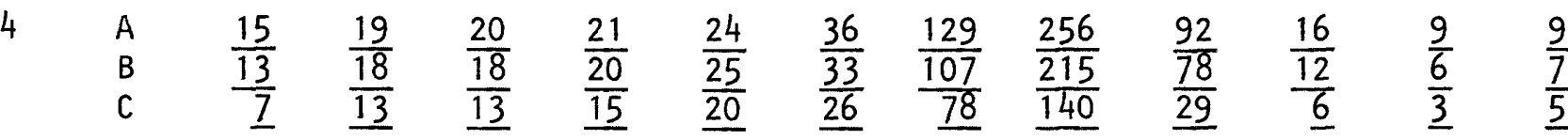

WITH TRANSMOUNTAIN DIVERSIONS

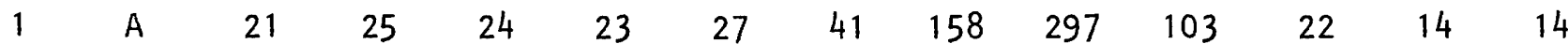

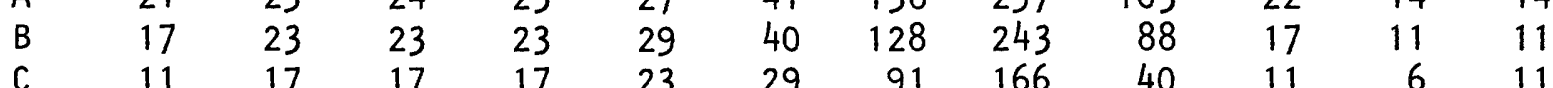

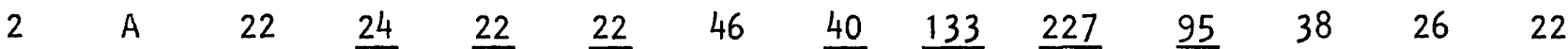

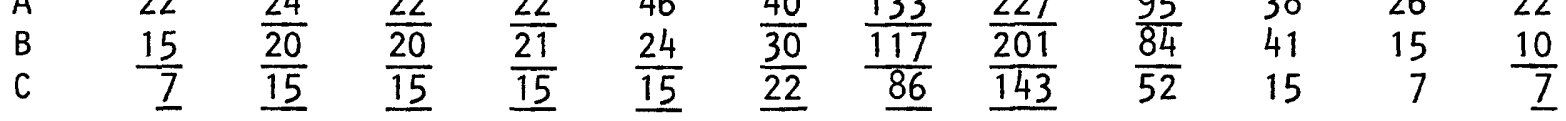

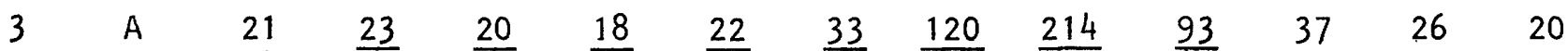

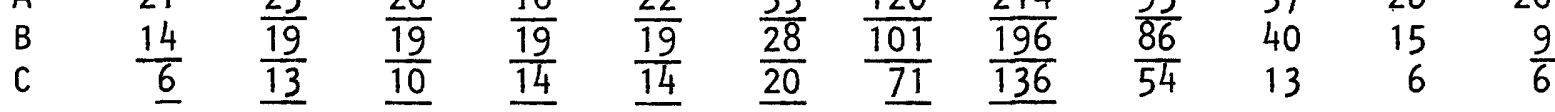

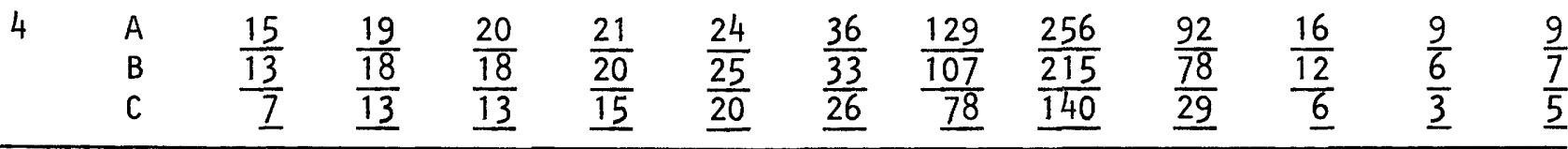


Table 21.--Summary of simulated historical monthly streamflows, control point 34 (Trout Creek at mouth),

with 100 percent of agricultural and no transmountain diversions, and with 100 percent of both agricultural and transmountain diversions, and including 100 percent of industrial and municipal diversions for all simulations

[FLOW VALUES: $A=M E A N ; B=M E D I A N ;$ and $C=80-P E R C E N T$ EXCEEDENCE. Underscored values are less than corresponding table 17 simulated historical conditions]

\begin{tabular}{l} 
OP- FLOW \\
\cline { 2 - 3 } TION VALUES \\
\cline { 2 - 4 } OCT. NOV. DEC. JAN. FEB. MAR. APR. MAY JUNE JULY AUG. SEPT
\end{tabular}

WITHOUT TRANSMOUNTAIN DIVERSIONS

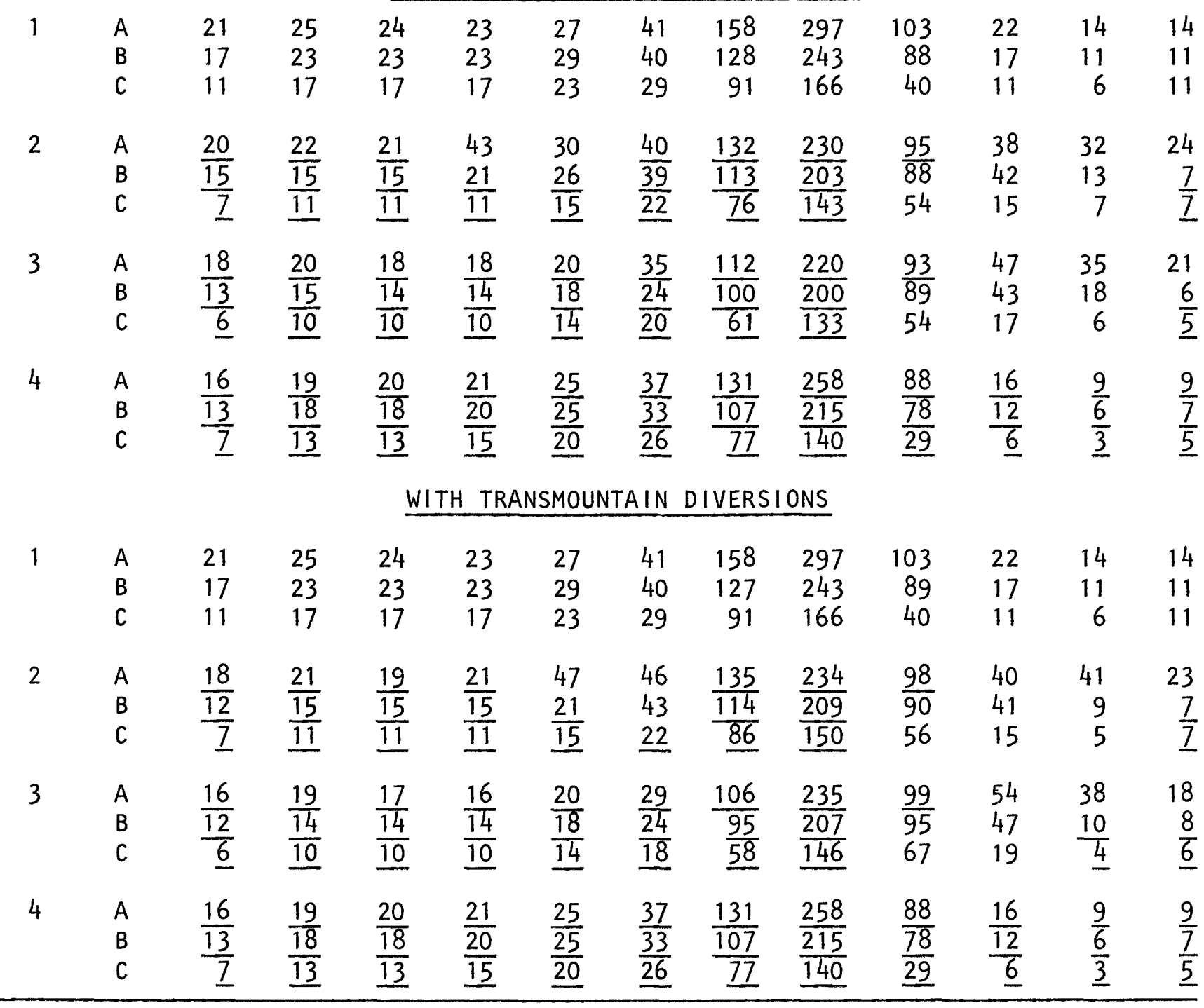


Model-simulated historical monthly streamflows for control point 28 (Yampa River at Craig, Colo.) are presented in tables 22 through 26. This control point is located downstream from the proposed Craig Reservoir and also downstream from the confluence of the Williams Fork (fig. 1). The simulated results for reservoirdevelopment option 1 represent both limited upstream reservoir development (table 3) and major downstream diversions from Juniper and Cross Mountain Reservoirs. The option-2 simulations included a larger number of upstream reservoirs (table 3) and consequently further reduced the flow at this site. Monthly streamflow simulations for reservoir-development options 3 and 4 included the immediate upstream effects of the Craig Reservoir and tended to even out the monthly flow distribution (tables 22 through 26). The monthly streamflow for reservoir-development option 4 is less than for option 3 because of the absence of the downstream demand from Juniper and Cross Mountain Reservoirs. The simulated historical 50-year mean monthly streamflows for the 100-percent water-use allocation (table 26) could be reduced to zero for at least 4 months each year under options 3 and 4 .

Table 22.--Summary of simulated historical monthly streamflows, control point 15 (Yampa River at Craig, Colo.),

for historical conditions and with 100 percent of transmountain diversions

[FLOW VALUES: A=MEAN; $B=M E D I A N$; and $C=80$-PERCENT EXCEEDENCE. Underscored values are less than simulated historical conditions without transmountain diversions]

FLOW

MONTHLY FLOWS, IN CUBIC FEET PER SECOND

VALUES OCT. NOV. DEC. JAN. FEB. MAR. APR. MAY JUNE JULY AUG. SEPT

SIMULATED HISTORICAL CONDITIONS

$\begin{array}{rrrrrrrrrrrrr}\text { A } & 138 & 266 & 273 & 211 & 260 & 531 & 2179 & 5052 & 4904 & 767 & 117 & 126 \\ \text { B } & 114 & 269 & 278 & 251 & 260 & 530 & 2193 & 5293 & 4924 & 477 & 89 & 129 \\ \text { C } & 72 & 210 & 244 & 98 & 245 & 505 & 1676 & 4036 & 3155 & 124 & 42 & 24\end{array}$

SIMULATED HISTORICAL CONDITIONS WITH 100 PERCENT OF TRANSMOUNTAIN DIVERSIONS

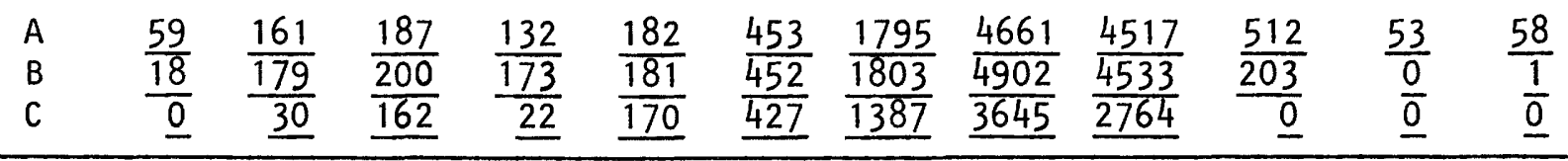


Table 23.--Summary of simulated historical monthly streamflows, control point 15 (Yampa River at Craig, Colo.),

with 25 percent of agricultural and no transmountain diversions, and with 25 percent of both agricultural and transmountain diversions, and including 100 percent of industrial and municipal diversions for all simulations

[FLOW VALUES: $A=M E A N ; B=M E D I A N ;$ and $C=80$-PERCENT EXCEEDENCE. Underscored values are less than corresponding table 22 simulated historical conditions]

OP- FLOW

MONTHLY FLOWS, IN CUBIC FEET PER SECOND

TION VALUES

OCT. NOV. DEC. JAN. FEB. MAR. APR. MAY JUNE JULY AUG. SEPT

WITHOUT TRANSMOUNTAIN DIVERSIONS

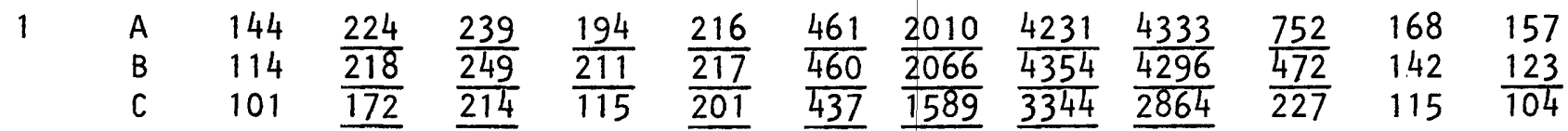

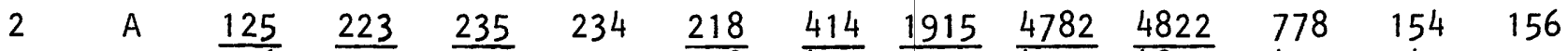

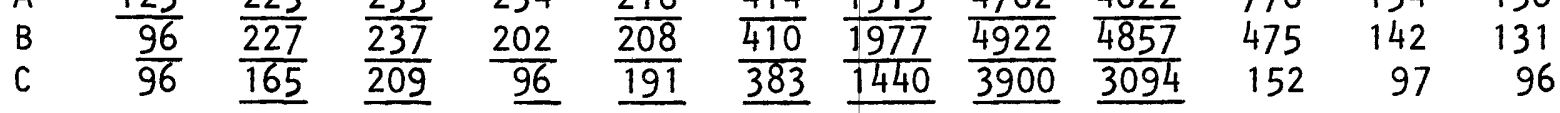

3 A 158

B 128

$\begin{array}{llll}271 & 308 & 254 & 290\end{array}$

$\frac{453}{460} \quad \frac{1807}{1884} \quad \frac{4214}{4315} \quad \frac{4542}{4517}$

$833 \quad 225 \quad 211$

C $\quad 115$

272309

289

291

$\overline{460} \overline{1884}$

4315

4517

$532 \quad 210$

169

$\begin{array}{lll}291 & 151 & 262\end{array}$

$\underline{422} \overline{1408} \quad \overline{3469} \quad \overline{2783}$

$274 \quad 155$

129

4

$\begin{array}{lll}\text { A } & \underline{32} & \frac{122}{128} \\ \text { B } & \frac{0}{0} & \underline{33}\end{array}$

$\frac{174}{186}$

$\frac{137}{170} \quad \frac{192}{194}$

$\frac{379}{\frac{377}{354}} \frac{1733}{\frac{1815}{1378}}$

$\frac{4534}{4627}$

$\frac{4732}{4740}$

$\frac{651}{\frac{343}{0}} \quad \frac{35}{\frac{3}{0}} \quad \frac{51}{13}$

WITH TRANSMOUNTAIN DIVERSIONS

1
$\begin{array}{ll}\text { A } & \frac{130}{108} \\ \text { B } & \frac{108}{100}\end{array}$
$\frac{199}{195}$
$\frac{214}{223}$
$\frac{176}{191} \quad \frac{193}{108} \quad \underline{173}$
$\frac{437}{439} \quad \frac{1913}{1970}$
$\frac{4124}{3225}$
$\frac{4232}{4194}$
$\frac{667}{380} \quad 154$
142
$\underline{185} \quad \frac{198}{108}$
$\underline{414} 1489$
$\frac{380}{131}$
124
110
$\frac{111}{100}$

2

$\begin{array}{llllllll}\text { A } & \frac{109}{96} & \frac{201}{206} & \frac{213}{213} & 212 & \frac{197}{180} & \frac{394}{186} & \frac{1815}{189} \\ \text { B } & \frac{969}{48} & \frac{186}{140} & \frac{186}{186} & \frac{60}{170} & \underline{364} & \frac{1344}{134}\end{array}$

4875

$\frac{4812}{3802} \quad \frac{4745}{2964}$

$\begin{array}{lll}702 & 145 & 142\end{array}$

$\begin{array}{rrr}\frac{780}{100} & 139 & \frac{121}{96}\end{array}$

3 A 144

$\frac{242}{241} 287$

$236 \quad 268$

$432 \quad 1701 \quad 4110$

4441

$754 \quad 210$

196

B 116

$\frac{241}{174} \quad 288$

$270 \quad 269$

$\frac{439}{402} \quad \frac{1766}{1277}$

$\frac{4194}{3378}$

$\underline{240} \quad \underline{402} \quad \underline{1277} \quad \underline{3378} \quad \underline{2720}$

$\frac{443}{216}$

198

155

$148 \quad 120$

4
$\begin{array}{lll}\text { A } & 24 \\ \text { C } & \end{array}$
$\begin{array}{lll}\frac{24}{0} & \frac{81}{58} & \frac{131}{164} \\ \underline{0} & \underline{43}\end{array}$
$\frac{115}{148} \quad \frac{169}{175}$
$\frac{360}{\frac{358}{335}} \frac{1635}{\frac{1718}{1281}} \quad \frac{4437}{\frac{4530}{3508}}$
$\frac{4634}{4642}$
$\begin{array}{lll}\frac{577}{245} & \frac{26}{0} & \frac{38}{0} \\ \frac{0}{0}\end{array}$ 
Table 24.--Sumary of simulated historical monthly streamflows, control point 15 (Yampa River at Craig, Colo.),

with 50 percent of agricultural and no transmountain diversions, and with 50 percent of both agricultural and transmountain diversions, and including 100 percent of industrial and municipal diversions for all simulations

[FLOW VALUES: $A=$ MEAN; $B=$ MEDIAN; and $C=80-P E R C E N T$ EXCEEDENCE. Underscored values are less than corresponding table 22 simulated historical conditions]

\begin{tabular}{|c|c|c|c|c|c|c|c|c|c|c|c|c|c|}
\hline \multirow{2}{*}{$\begin{array}{l}\text { OP- } \\
\text { TION }\end{array}$} & \multirow{2}{*}{$\begin{array}{c}\text { FLOW } \\
\text { VALUES }\end{array}$} & \multicolumn{12}{|c|}{ MONTHLY FLOWS, IN CUBIC FEET PER SECOND } \\
\hline & & OCT. & NOV. & DEC. & JAN. & FEB. & MAR. & APR. & MAY & JUNE & JULY & AUG. & SEPT \\
\hline
\end{tabular}

WITHOUT TRANSMOUNTAIN DIVERSIONS

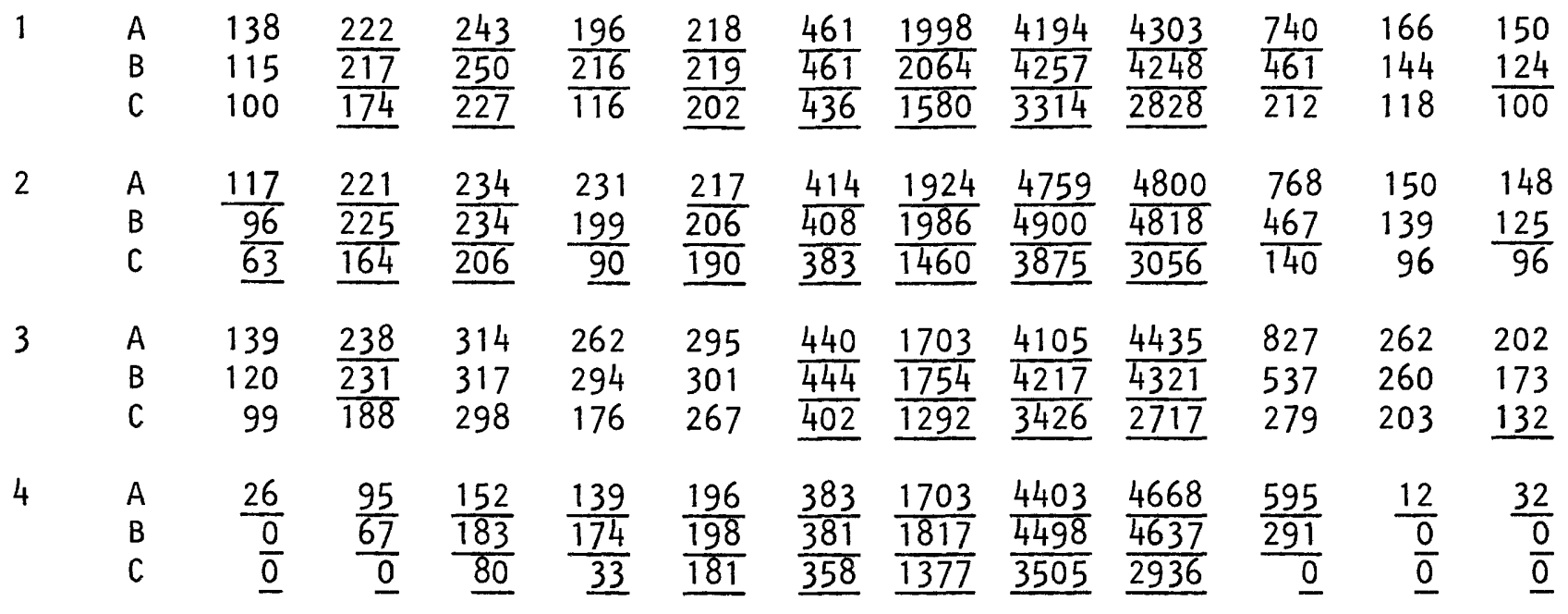
WITH TRANSMOUNTAIN DIVERSIONS

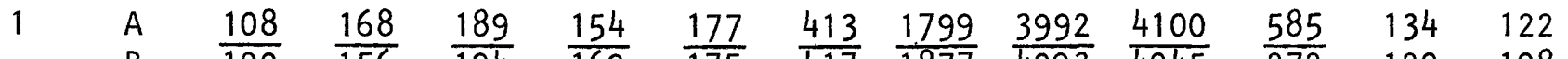

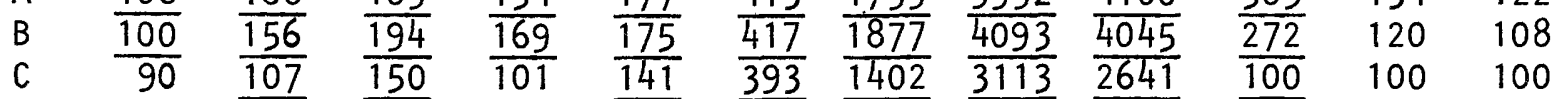

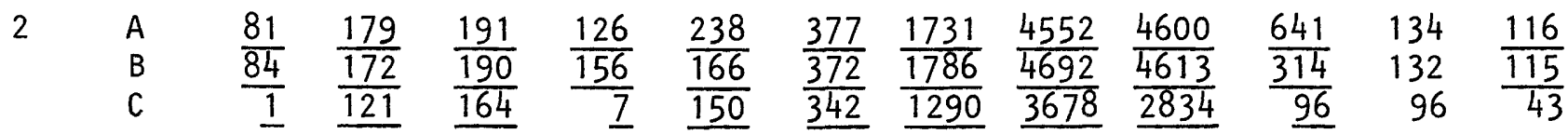

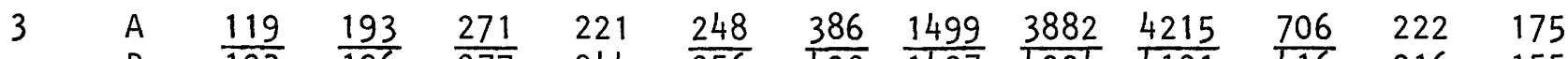

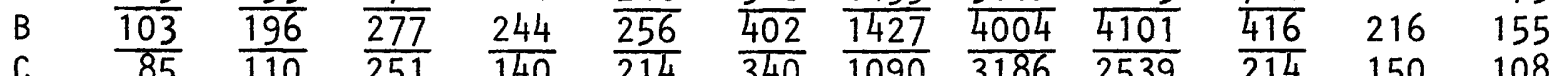

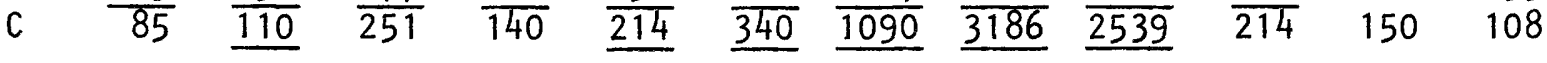

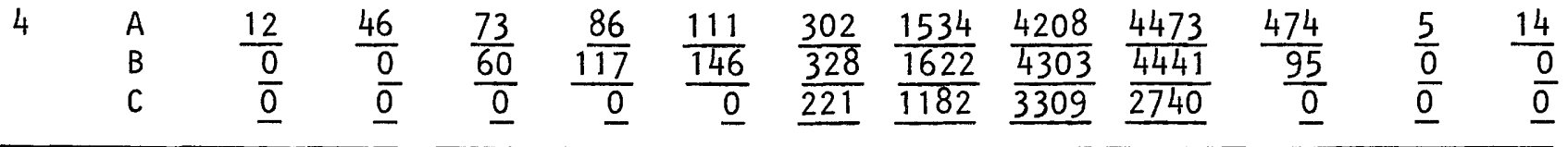


Table 25.--Summary of simulated historical monthly streamflows, control point 15 (Yompa River at Craig, Colo.),

with 75 percent of agricultural and no transmountain diversions, and with 75 percent of both agricultural and transmountain diversions, and including 100 percent of industrial and municipal diversions for all simulations

[FLOW VALUES: $A=$ MEAN; $B=$ MEDIAN; and $C=80$-PERCENT EXCEEDENCE. Underscored values are less than corresponding table 22 simulated historical conditions]

\begin{tabular}{cccccc}
\hline MONTHLY FLOWS, IN CUBIC FEET PER SECOND \\
TION VALUES \\
\cline { 2 - 5 } & OCT. NOV. DEC. JAN. FEB. MAR. APR. MAY JUNE JULY AUG. SEPT \\
\hline
\end{tabular}

WITHOUT TRANSMOUNTAIN DIVERSIONS

$\begin{array}{llllllllll}1 & A & 138 & 222 & 243 & 196 & 218 & 461 & 1998 & 4194\end{array}$

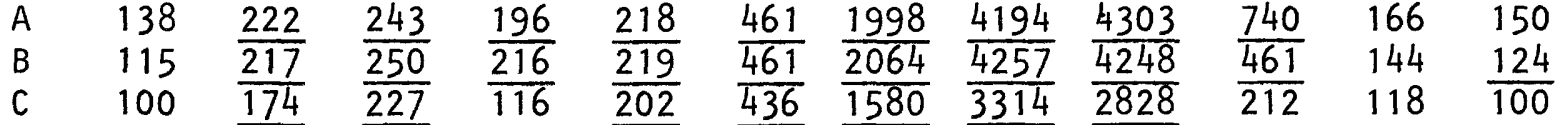

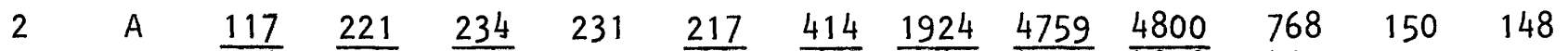

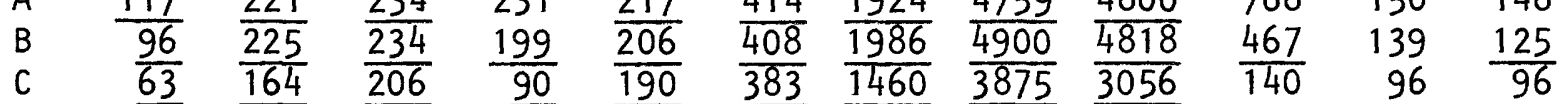

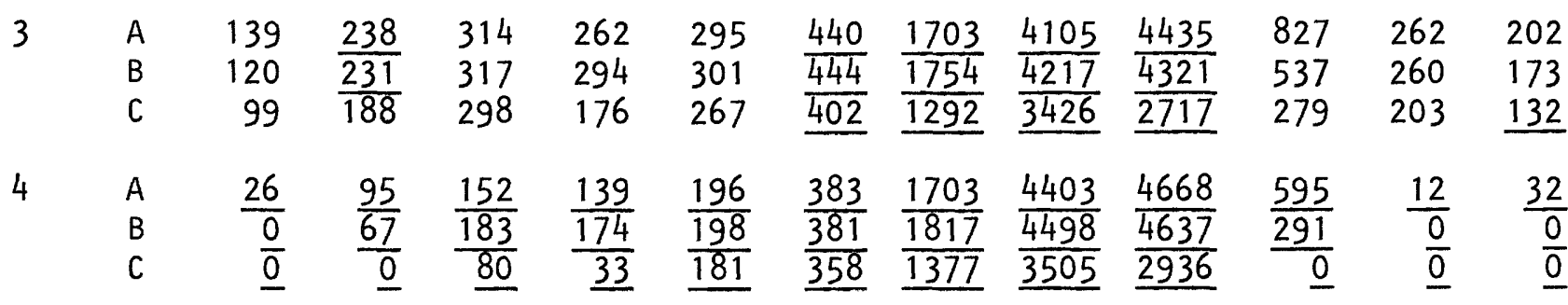

WITH TRANSMOUNTAIN DIVERSIONS

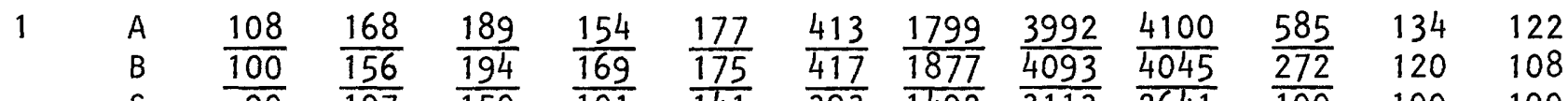

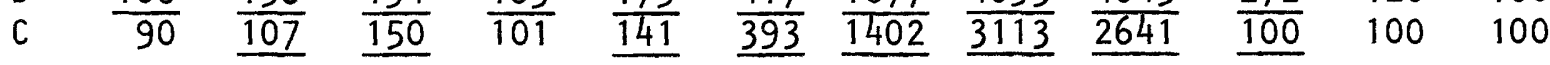

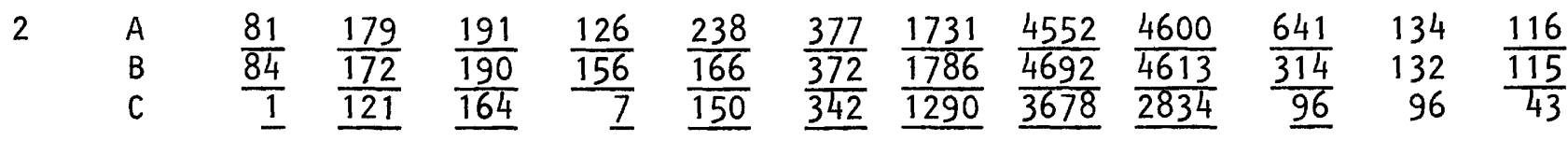

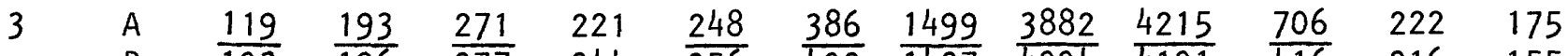

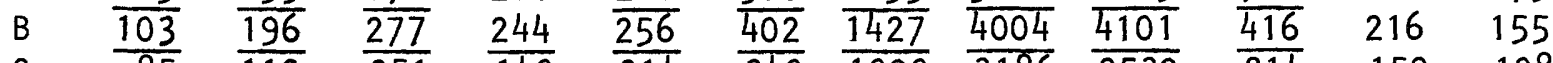

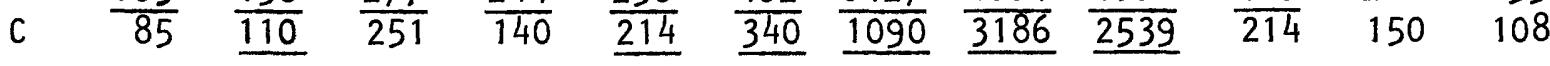

$\begin{array}{llllllllllllll}\text { A } & \text { A } & \frac{12}{0} & \frac{46}{0} & \frac{73}{60} & \frac{86}{17} & \frac{111}{146} & \frac{302}{328} & \frac{1534}{1622} & \frac{4208}{4303} & \frac{4473}{4441} & \frac{474}{95} & \frac{5}{0} & \frac{14}{0} \\ \text { B } & \underline{0} & \underline{0} & \frac{0}{0} & \frac{0}{0} & \frac{0}{0} & \underline{0} & \underline{1182} & \underline{3309} & \underline{2740} & \underline{0} & \underline{0} & \underline{0}\end{array}$ 
Table 26.--Sumary of simulated historical monthly streamflows, control point 15 (Yampa River at Craig, Colo.),

with 100 percent of agricultural and no transmountain diversions, and with 100 percent of both agricultural and transmountain diversions, and including 100 percent of industrial and municipal diversions for all simulations

[FLOW VALUES: $A=M E A N ; B=M E D I A N$; and $C=80-P E R C E N T$ EXCEEDENCE. Underscored values are less than corresponding table 22 simulated historical conditions]

\begin{tabular}{|c|c|c|c|c|c|c|c|c|c|c|c|c|c|}
\hline \multirow{2}{*}{$\begin{array}{l}\text { OP- } \\
\text { TION }\end{array}$} & \multirow{2}{*}{$\begin{array}{c}\text { FLOW } \\
\text { VALUES }\end{array}$} & \multicolumn{12}{|c|}{ MONTHLY FLOWS, IN CUBIC FEET PER SECOND } \\
\hline & & OCT. & NOV. & DEC. & JAN. & FEB. & MAR. & APR. & MAY & JUNE & JULY & AUG. & SEPT \\
\hline & \multicolumn{13}{|c|}{ WITHOUT } \\
\hline 1 & $\begin{array}{l}A \\
B \\
C\end{array}$ & $\begin{array}{l}87 \\
61 \\
25\end{array}$ & $\begin{array}{l}194 \\
197 \\
132\end{array}$ & $\begin{array}{l}211 \\
193 \\
176\end{array}$ & $\begin{array}{l}201 \\
185 \\
115\end{array}$ & $\begin{array}{l}249 \\
218 \\
158\end{array}$ & $\begin{array}{l}447 \\
424 \\
381\end{array}$ & $\begin{array}{l}2038 \\
2093 \\
1680\end{array}$ & $\begin{array}{l}4209 \\
4340 \\
3330\end{array}$ & $\begin{array}{l}4271 \\
4232 \\
2843\end{array}$ & $\begin{array}{l}732 \\
459 \\
203\end{array}$ & $\begin{array}{r}124 \\
113 \\
34\end{array}$ & $\begin{array}{r}104 \\
79 \\
19\end{array}$ \\
\hline 2 & $\begin{array}{l}A \\
B \\
C\end{array}$ & $\begin{array}{r}62 \\
14 \\
0\end{array}$ & $\begin{array}{l}181 \\
176 \\
119\end{array}$ & $\begin{array}{l}207 \\
200 \\
176\end{array}$ & $\begin{array}{r}204 \\
173 \\
39\end{array}$ & $\begin{array}{l}200 \\
189 \\
162\end{array}$ & $\begin{array}{l}395 \\
400 \\
334\end{array}$ & $\begin{array}{l}1884 \\
2014 \\
1408\end{array}$ & $\begin{array}{l}4765 \\
4963 \\
3884\end{array}$ & $\begin{array}{l}4780 \\
4809 \\
3067\end{array}$ & $\begin{array}{l}772 \\
465 \\
154\end{array}$ & $\begin{array}{r}128 \\
127 \\
6\end{array}$ & $\begin{array}{r}107 \\
66 \\
0\end{array}$ \\
\hline 3 & $\begin{array}{l}A \\
B \\
C\end{array}$ & $\frac{56}{\frac{0}{0}}$ & $\frac{65}{\frac{0}{0}}$ & $\frac{76}{\frac{0}{0}}$ & $\frac{89}{\frac{0}{0}}$ & $\frac{94}{0}$ & $\frac{\frac{304}{189}}{16}$ & $\frac{\frac{1658}{1690}}{1180}$ & $\frac{\frac{4464}{4624}}{3624}$ & $\frac{4516}{\frac{4521}{3145}}$ & $\begin{array}{l}997 \\
753 \\
438\end{array}$ & $\begin{array}{r}272 \\
304 \\
33 \\
\end{array}$ & $\begin{array}{r}133 \\
\underline{0} \\
0\end{array}$ \\
\hline 4 & $\begin{array}{l}A \\
B \\
C\end{array}$ & $\frac{11}{\frac{0}{0}}$ & $\frac{36}{\frac{0}{0}}$ & $\frac{\frac{83}{25}}{0}$ & $\frac{\frac{116}{166}}{0}$ & $\frac{\frac{151}{195}}{0}$ & $\frac{\frac{370}{375}}{335}$ & $\frac{\frac{1724}{1813}}{1370}$ & $\frac{\frac{4276}{4374}}{3329}$ & $\frac{\frac{4548}{4519}}{2828}$ & $\frac{\frac{483}{113}}{0}$ & $\frac{0}{0}$ & $\frac{0}{0}$ \\
\hline
\end{tabular}

WITH TRANSMOUNTAIN DIVERSIONS

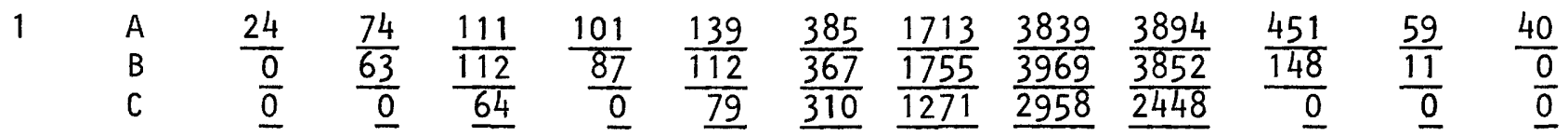

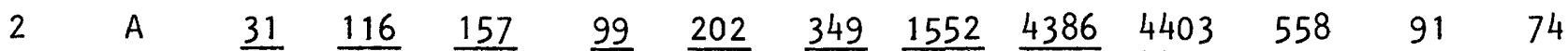

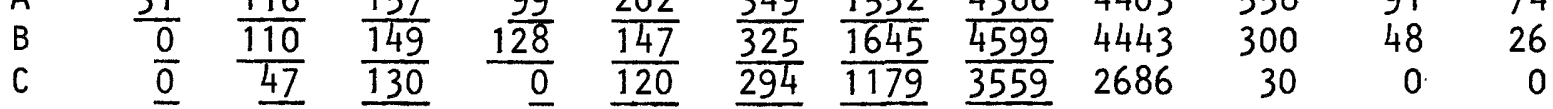

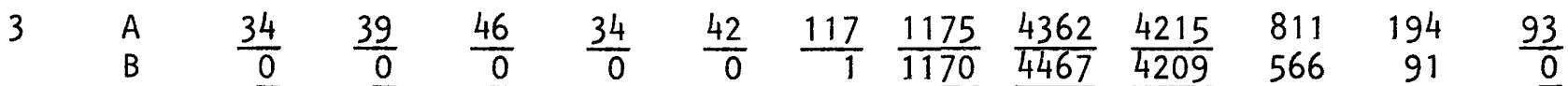

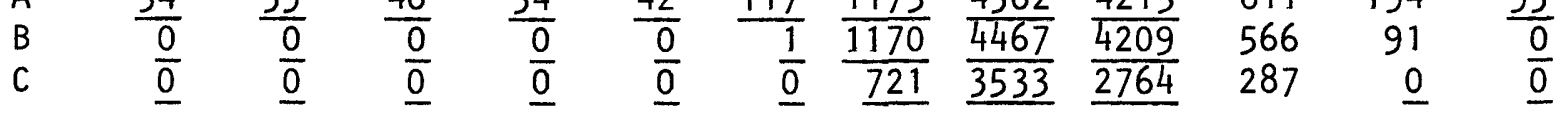

$\begin{array}{llllllllllllll}4 & \text { A } & \underline{0} & \frac{6}{0} & \underline{9} & \frac{14}{0} & \frac{26}{0} & \frac{131}{91} & \frac{1274}{1408} & \frac{3885}{3983} & \frac{4160}{4128} & \underline{301} & \frac{0}{0} & \frac{0}{0} \\ \text { B } & \underline{0} & \underline{0} & \underline{0} & \underline{0} & \underline{0} & \frac{0}{0} & \frac{9}{031} & \frac{2938}{2437} & \underline{0} & \frac{0}{0} & \frac{1}{0}\end{array}$ 
The upstream Vidler transmountain diversion could have varying effects (tables 22 through 26) on the flow at Craig. For the historical condition with 100 percent of transmountain diversions (table 22), the 5-year low flow (80-percent exceedence probability) could decrease to zero for 3 months. Simulated historical monthly streamflows at the 100-percent water-use allocation (table 26) could also be significantly reduced by inclusion of the vidler transmountain diversion.

Model-simulated historical monthly streamflows for control point 25 (confluence of Yampa River and Milk (reek) are presented in tables 27 through 31 . This site is located approximately 10 river miles upstream from the damsite of the proposed Juniper Reservoir (fig. 1).

At the confluence of the Yampa River and Milk Creek, the flow statistics are similar to the upstream Yampa River at Craig, Colo. (control point 15), with only the addition of flow from Milk Creek and return flow from Craig Reservoir diversions. Reservoir-development options 1 and 2 were similar in effect, with less mean annual flow for option 2 due to additional reservoir storage upstream. For reservoir-development option 3, the larger flow statistics reflect the Juniper and Cross Mountain Reservoir downstream demands. In reservoir-development option 4, the downstream demands were nonexistent, and the flow statistics decreased at this site. There also was a decrease in flow statistics as the allocation percentages increased (tables 28 through 31), but to a much smaller degree. The absence of a downstream demand could allow more water to be retained in Craig Reservoir and other upstream reservoirs and less water to be released.

\section{Table 27.--Summary of simulated historical monthly streamflows, control point 25 (confluence of Yampa River and Milk Creek), for simulated historical conditions and with 100 percent of transmountain diversions}

[FLOW VALUES: $A=M E A N ; B=M E D I A N ;$ and $C=80-P E R C E N T$ EXCEEDENCE. Underscored values are less than simulated historical conditions without transmountain diversions]

\begin{tabular}{|c|c|c|c|c|c|c|c|c|c|c|c|c|}
\hline \multirow{2}{*}{$\begin{array}{c}\text { FLOW } \\
\text { VALUES }\end{array}$} & \multicolumn{12}{|c|}{ MONTHLY FLOWS, IN CUBIC FEET PER SECOND } \\
\hline & OCT. & NOV. & DEC. & JAN. F & FEB. 1 & MAR. & APR. & MAY & JUNE & JULY & AUG. & SEPT \\
\hline \multicolumn{13}{|c|}{ SIMULATED HISTORICAL CONDITIONS } \\
\hline $\begin{array}{l}A \\
B \\
C\end{array}$ & $\begin{array}{r}150 \\
122 \\
76\end{array}$ & $\begin{array}{l}278 \\
279 \\
218\end{array}$ & $\begin{array}{l}286 \\
289 \\
259\end{array}$ & $\begin{array}{l}223 \\
267 \\
109\end{array}$ & $\begin{array}{l}275 \\
273 \\
261\end{array}$ & $\begin{array}{l}564 \\
565 \\
528\end{array}$ & $\begin{array}{l}2349 \\
2373 \\
1763\end{array}$ & $\begin{array}{l}5571 \\
5696 \\
4409\end{array}$ & $\begin{array}{l}5088 \\
4996 \\
3240\end{array}$ & $\begin{array}{l}794 \\
498 \\
147\end{array}$ & $\begin{array}{r}125 \\
99 \\
43\end{array}$ & $\begin{array}{r}133 \\
133 \\
32\end{array}$ \\
\hline \multicolumn{2}{|c|}{ SIMULATED } & HISTORI & CAL CO & IDITIONS & S WITH & 100 & PERCENT & $\mathrm{TOF}$ & TRANSMO & UNTAIN & DIVER & SIONS \\
\hline $\begin{array}{l}A \\
B \\
C\end{array}$ & $\frac{\frac{71}{26}}{\frac{4}{4}}$ & $\frac{\frac{174}{195}}{38}$ & $\frac{\frac{200}{211}}{170}$ & $\frac{\frac{144}{189}}{33}$ & $\frac{\frac{197}{194}}{183}$ & $\frac{\frac{486}{487}}{450}$ & $\frac{1965}{\frac{1982}{1451}}$ & $\frac{\frac{5180}{5305}}{4018}$ & $\frac{\frac{4701}{4605}}{2849}$ & $\frac{\frac{539}{235}}{11}$ & $\frac{\frac{62}{23}}{4}$ & $\frac{\frac{65}{18}}{4}$ \\
\hline
\end{tabular}


Table 28.--Sumary of simulated historical monthly streamflows, control point 25 (confluence of Yampa River and Milk Creek), with 25 percent of agricultural and no transmountain diversions, and with 25 percent of both agricultural and transmountain diversions, and including 100 percent of industrial and municipal diversions for all simulations

[FLOW VALUES: $A=M E A N ; B=M E D I A N ;$ and $C=80-P E R C E N T$ EXCEEDENCE. Underscored values are less than corresponding table 27 simulated historical conditions]

\begin{tabular}{llllll} 
OP- FLOW \\
\cline { 2 - 3 } TION VALUES & \multicolumn{5}{c}{ MONTHLY FLOWS, IN CUBIC FEET PER SECOND } \\
\hline
\end{tabular}

WITHOUT TRANSMOUNTAIN DIVERSIONS

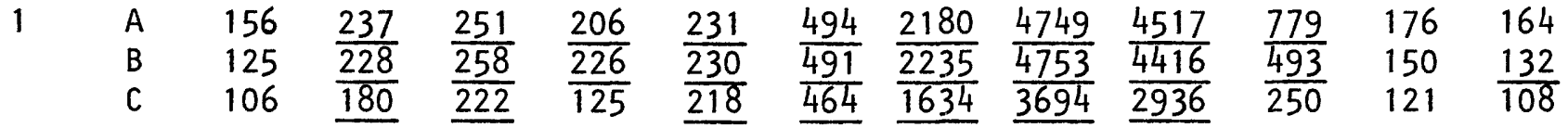

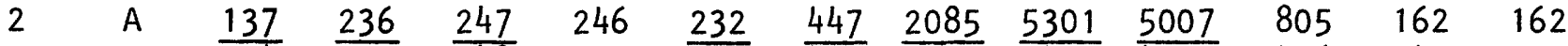

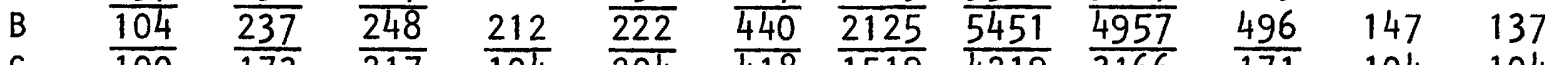

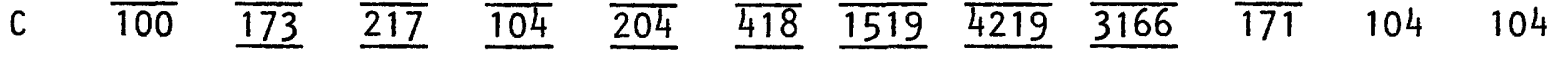

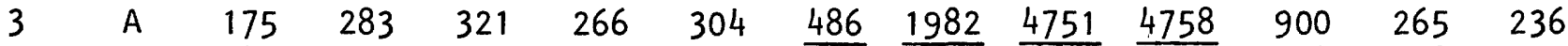

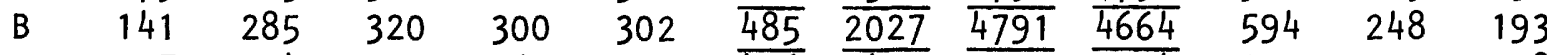

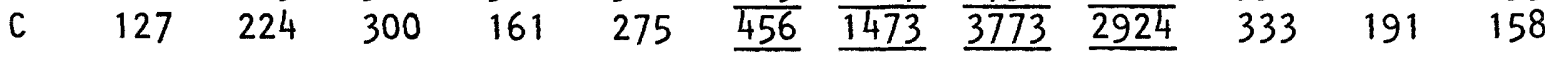
$\begin{array}{lllllllllllll}\text { A } & \frac{48}{15} & \frac{136}{138} & \frac{187}{200} & \frac{149}{189} & \frac{207}{206} & \frac{413}{410} & \frac{1908}{1945} & \frac{5071}{5015} & \frac{4947}{4859} & \frac{718}{404} & \frac{75}{46} & \frac{76}{44} \\ \text { B } & \frac{15}{8} & \frac{41}{162} & \frac{41}{188} & \underline{382} & \underline{1457} & \underline{3917} & \underline{3113} & \frac{613}{63} & \underline{35} & \underline{22}\end{array}$

WITH TRANSMOUNTAIN DIVERSIONS

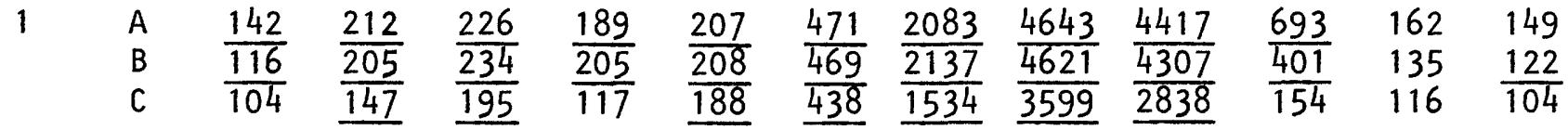

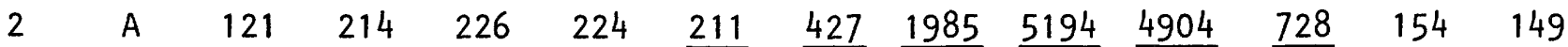

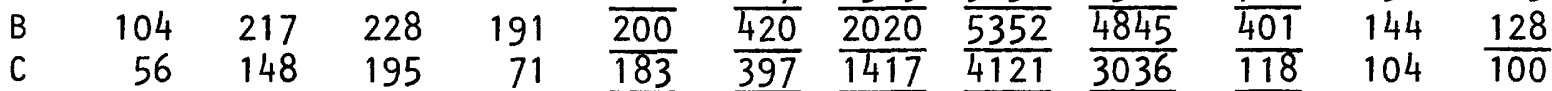

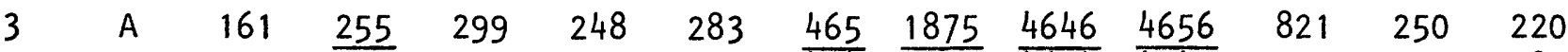

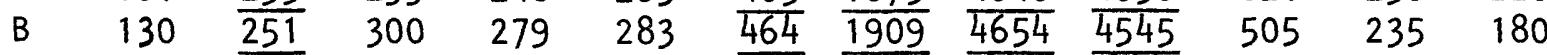

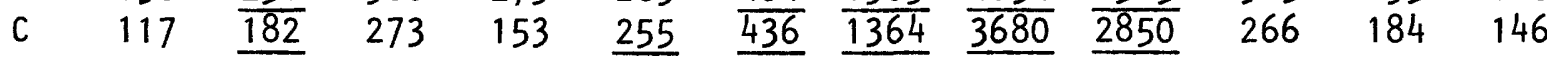

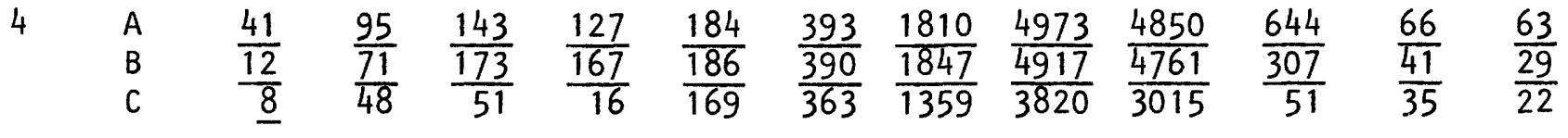


Table 29.--Summary of simulated historical monthly streamflows, control point 25 (confluence of Yampa River and Milk Creek), with 50 percent of agricultural and no transmountain diversions, and with 50 percent of both agricultural and transmountain diversions, and including 100 percent of industrial and municipal diversions for all simulations

[FLOW VALUES: $A=M E A N ; B=M E D I A N ;$ and $C=80-P E R C E N T$ EXCEEDENCE. Underscored values are less than corresponding table 27 simulated historical conditions]

OP- FLOW

MONTHLY FLOWS, IN CUBIC FEET PER SECOND

\section{TION VALUES}

OCT. NOV. DEC. JAN. FEB. MAR. APR. MAY JUNE JULY AUG. SEPT

WITHOUT TRANSMOUNTAIN DIVERSIONS

1 A 150

B 124

C 105

\section{$\begin{array}{llllll}\frac{256}{261} & \frac{208}{231} & \frac{232}{232} & \frac{494}{490} & \frac{2166}{2232} & 4713\end{array}$}

$\overline{490} \quad \frac{2166}{1625}$

$\underline{465} \quad \frac{2232}{1625}$

3691

$\underline{238}$

$\underline{217}$

$\frac{447}{439} \quad \frac{2094}{2116}$

$\frac{5278}{5439}$

$\underline{417} \quad \underline{1534} \quad \underline{4214}$

4488

4379

2900

$767 \quad 175$

157

482

149

122

132

$2 \quad A$

$\begin{array}{llll}\text { A } & \frac{128}{104} & \frac{233}{236} & \frac{246}{247} \\ \text { C } & \frac{244}{172} & \underline{215}\end{array}$

3

$\begin{array}{ll}\text { A } & 159 \\ B & 139 \\ \text { C } & 116\end{array}$

$\frac{250}{247}$

327

274

310

47

$\frac{1882}{\frac{1944}{1346}}$

$\frac{4659}{3662}$

$4985 \quad 795$

159

$\frac{4921}{3142}$

488

144

104

155

$\begin{array}{lll}312 & 186 & 282\end{array}$

$\underline{433} \quad \underline{1346}$

$\frac{4682}{4521}$

934

333

330

244

374

270

217

172

$4 \quad \mathrm{~A}$
$\begin{array}{llll}\text { A } & \frac{47}{17} & \frac{110}{85} & \frac{164}{195} \\ \text { C } & \underline{13} & \frac{8}{90}\end{array}$

$\frac{151}{190} \quad \frac{210}{210}$

$\frac{417}{414} \quad \frac{1909}{1949} \quad \frac{4957}{4932} \quad \frac{4915}{4785}$

$\overline{386} \quad \frac{1464}{3846}$

$\frac{\frac{702}{392}}{92}$

$\frac{83}{70} \quad \frac{74}{46}$

WITH TRANSMOUNTAIN DIVERSIONS

1 A
$\begin{array}{ll}\text { A } & \frac{120}{111} \\ \text { C } & \frac{119}{98}\end{array}$
$\frac{181}{169}$
$\frac{\frac{201}{206}}{161} \quad \frac{166}{185} \quad \frac{192}{111} \quad \frac{188}{152}$
$\frac{\frac{446}{446}}{412} \quad \frac{1969}{2034}$
$\frac{4511}{4488}$
$\frac{4284}{4172}$

$\frac{612}{293}$

142

130

104

128

2 A

$$
\begin{array}{llllll}
\text { A } & \frac{93}{101} & \frac{192}{184} & \frac{204}{204} & \frac{138}{171} & \frac{252}{180} \\
\text { C } & \frac{10}{10} & \frac{129}{175} & \frac{16}{165} &
\end{array}
$$

$\begin{array}{llll}\frac{410}{400} & \frac{1901}{1906} & \frac{5071}{5253} & \frac{4785}{4756} \\ \underline{4018} & \frac{4722}{2955}\end{array}$

$\begin{array}{ll}\frac{668}{335} & 143 \\ 134\end{array}$

$\frac{335}{112} \quad 100$

123

813

293

217

$\begin{array}{llllll}\text { A } & \frac{140}{121} & \frac{205}{210} & \frac{283}{287} & 233 & \frac{263}{125} \\ \text { B } & \frac{267}{102} & \frac{267}{126} & \frac{267}{150} & \underline{232}\end{array}$

$419 \quad \frac{1678 \quad 4436}{4656} \quad 4462$

518

285

200

$\frac{428}{372} \quad \frac{1656}{1258} \quad \frac{4418}{3500} \quad \frac{4289}{2789}$

306

213

158

$4 \quad A$
A
$B$
$C$
\begin{tabular}{lll}
$\frac{33}{17}$ & $\frac{61}{11}$ & $\frac{86}{75}$ \\
\hline
\end{tabular}
$\frac{\frac{98}{134}}{11} \quad \frac{125}{160}$
$\begin{array}{llll}\frac{335}{365} & \frac{1713}{251} & \frac{4762}{1268} & \frac{4736}{4650}\end{array} \quad \frac{\frac{4720}{4589}}{2882}$
$\begin{array}{lll}\frac{582}{197} & \frac{76}{92} & \frac{56}{61} \\ \underline{66} & \underline{40}\end{array}$ 
Table 30.--Summary of simulated historical monthly streamflows, control point 25 (confluence of Yampa River and Milk Creek), with 75 percent of agricultural and no transmountain diversions, and with 75 percent of both agricultural and transmountain diversions, and including 100 percent of industrial and municipal diversions for all simulations

[FLOW VALUES: $A=M E A N ; B=M E D I A N ;$ and $C=80-P E R C E N T$ EXCEEDENCE. Underscored values are less than corresponding table 27 simulated historical conditions]

OP- FLOW

MONTHLY FLOWS, IN CUBIC FEET PER SECOND

TION VALUES

OCT. NOV. DEC. JAN. FEB. MAR. APR. MAY JUNE JULY AUG. SEPT

WITHOUT TRANSMOUNTAIN DIVERSIONS

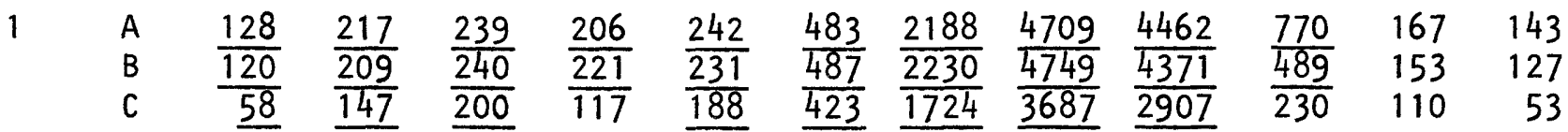

2

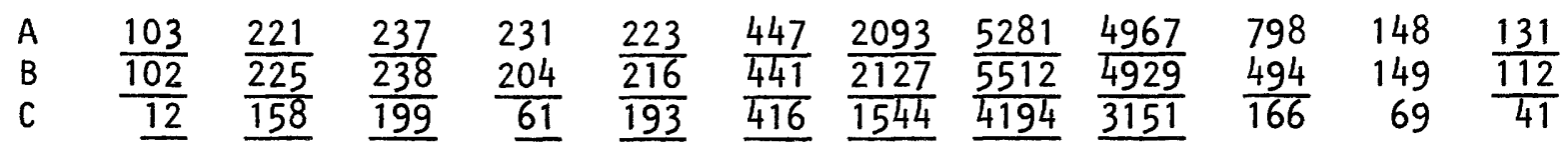

$\begin{array}{rrrrrrrrrrrrr}\text { A } & 132 & 177 & 210 & 179 & 203 & 488 & 1782 & \underline{4789} & \frac{4660}{460} & 1071 & 402 & 263 \\ \text { B } & 143 & 181 & 266 & 203 & 239 & 432 & \underline{1802} & \underline{5042} & \underline{4606} & 869 & 413 & 273 \\ \text { C } & 21 & 11 & 11 & 11 & 11 & 158 & \underline{1341} & \underline{3661} & \underline{3090} & 518 & 339 & 150\end{array}$

4
$\begin{array}{lll}\text { A } & \frac{41}{2} \\ \text { B } & \frac{2}{17} \\ \text { C }\end{array}$
$\frac{\frac{41}{21}}{17} \quad \frac{76}{13}$
$\frac{76}{\frac{13}{8}} \quad \frac{128}{151} \quad \frac{139}{11} \quad \frac{192}{11}$
$\frac{191}{\frac{211}{157}}$
$\begin{array}{llll}\frac{419}{414} & \frac{1910}{1952} & \frac{4912}{14890} & \frac{4888}{3779}\end{array}$
\begin{tabular}{rrr}
$\frac{687}{362}$ & $\frac{104}{102}$ & $\frac{71}{57}$ \\
\hline 132 & 98 & $\frac{57}{57}$
\end{tabular}

WITH TRANSMOUNTAIN DIVERSIONS

$1 \quad A$
A
$B$
C
$\frac{\frac{58}{40}}{\underline{0}} \quad \frac{123}{120}$
$\frac{147}{136} \quad \frac{111}{113}$
$\frac{159}{139}$
$\frac{\frac{409}{389}}{353} \quad \frac{1763}{1804}$
$\frac{3911}{4033}$
$\frac{3989}{3943}$
$\begin{array}{lll}\frac{514}{191} & \frac{96}{92}\end{array} \quad \frac{76}{24}$

2
$A$
$B$
$C$
$\frac{63}{\frac{63}{4}} \quad \frac{159}{159}$
$\frac{\frac{183}{180}}{153} \quad \frac{122}{158} \quad \frac{232}{11} \quad \frac{162}{136}$

$\frac{\frac{384}{377}}{337} \quad \frac{1826}{1823}$

$\frac{4983}{5171}$

$\frac{4684}{4672}$

$\frac{624}{333} \quad \frac{110}{106}$

$\frac{92}{76}$

3
$A$
$B$
$C$
$\frac{97}{94} \quad 126$
$\frac{148}{151} \quad \frac{118}{91}$
$\frac{118}{\frac{91}{11}} \quad \frac{141}{140}$
$\frac{276}{346} \quad \frac{1616}{1537} \quad \frac{4544}{4850} \quad \frac{4424}{4423}$
$\begin{array}{lll}916 & 336 & 201\end{array}$
$\begin{array}{lll}663 & 347 \quad 202\end{array}$
$\frac{94}{9} \quad \frac{93}{11}$
$\underline{11} \quad \underline{11}$
30 1089
$\underline{3334} \quad 2926$
$426 \quad 201$
$\underline{25}$

4
$\begin{array}{lll}\text { A } & \frac{32}{21} & \frac{27}{11} \\ \text { B } & \frac{21}{17} & \frac{8}{6}\end{array}$
$\frac{42}{\frac{11}{8}}$
$\frac{51}{\frac{11}{8}}$

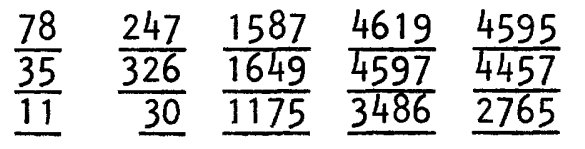
$\frac{\frac{532}{144}}{\frac{132}{132}} \quad \frac{102}{98} \quad \frac{61}{57}$ 
Table 31.--Summary of simulated historical monthly streamflows, control point 25 (confluence of Yampa River and Milk Creek), with 100 percent of agricultural and no transmountain diversions, and with 100 percent of both agricultural and transmountain diversions, and including 100 percent of industrial and municipal diversions for all simulations

[FLOW VALUES: $A=M E A N ; B=M E D I A N ;$ and $C=80-P E R C E N T$ EXCEEDENCE. Underscored values are less than corresponding table 27 simulated historical conditions]

\begin{tabular}{l} 
MONTHLY FLOWS, IN CUBIC FEET PER SECOND \\
TION VALUES \\
\cline { 2 - 4 } OCT. NOV. DEC. JAN. FEB. MAR. APR. MAY JUNE JULY AUG. SEPT
\end{tabular}

WITHOUT TRANSMOUNTAIN DIVERSIONS

\begin{tabular}{|c|c|c|c|c|c|c|c|c|c|c|c|c|c|}
\hline 1 & $\begin{array}{l}A \\
B \\
C\end{array}$ & $\frac{99}{\frac{75}{32}}$ & $\frac{\frac{207}{213}}{140}$ & $\frac{\frac{223}{203}}{185}$ & $\frac{\frac{213}{201}}{126}$ & $\frac{\frac{263}{233}}{171}$ & $\begin{array}{l}\frac{480}{450} \\
409\end{array}$ & $\frac{\frac{2208}{2261}}{1725}$ & $\frac{4728}{4745} \frac{3727}{4}$ & $\frac{\frac{4456}{4363}}{2915}$ & $\frac{\frac{758}{480}}{222}$ & $\begin{array}{r}132 \\
119 \\
37 \\
\end{array}$ & $\frac{111}{\frac{86}{24}}$ \\
\hline 2 & $\begin{array}{l}A \\
B \\
C\end{array}$ & $\frac{\frac{74}{28}}{4}$ & $\frac{\frac{193}{191}}{127}$ & $\frac{\frac{220}{212}}{187}$ & $\frac{\frac{216}{183}}{43}$ & $\frac{\frac{214}{203}}{174}$ & $\frac{428}{433}$ & $\frac{\frac{2054}{2134}}{1524}$ & $\frac{\frac{5284}{5477}}{4180}$ & $\frac{\frac{4964}{4936}}{3152}$ & $\begin{array}{l}799 \\
486 \\
173\end{array}$ & $\begin{array}{r}137 \\
131 \\
17 \\
\end{array}$ & $\frac{114}{74}$ \\
\hline 3 & $\begin{array}{l}A \\
B \\
C\end{array}$ & $\frac{\frac{83}{26}}{10}$ & $\frac{\frac{78}{11}}{8}$ & $\frac{\frac{88}{11}}{11}$ & $\frac{101}{\frac{15}{8}}$ & $\frac{109}{\frac{17}{11}}$ & $\frac{\frac{337}{222}}{53}$ & $\frac{\frac{1846}{1858}}{1277}$ & $\frac{5054}{\frac{5215}{3959}}$ & $\frac{\frac{4825}{4785}}{3390}$ & $\begin{array}{r}1177 \\
937 \\
607\end{array}$ & $\begin{array}{r}376 \\
437 \\
65\end{array}$ & $\begin{array}{r}181 \\
60 \\
8\end{array}$ \\
\hline 4 & $\begin{array}{l}A \\
B \\
C\end{array}$ & $\frac{\frac{40}{26}}{22}$ & $\frac{\frac{51}{11}}{8}$ & $\frac{\frac{96}{38}}{11}$ & $\frac{\frac{128}{183}}{11}$ & $\frac{\frac{166}{209}}{11}$ & $\frac{\frac{403}{412}}{368}$ & $\frac{\frac{1912}{1956}}{1469}$ & $\frac{4866}{\frac{4868}{3772}}$ & $\frac{\frac{4857}{4723}}{3025}$ & $\frac{\frac{671}{296}}{172}$ & $\begin{array}{l}133 \\
133 \\
129\end{array}$ & $\frac{78}{75}$ \\
\hline
\end{tabular}

WITH TRANSMOUNTAIN DIVERSIONS

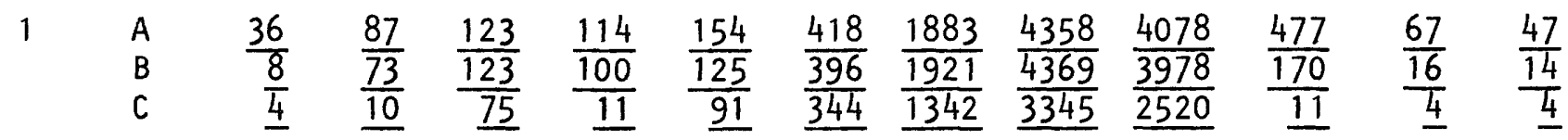

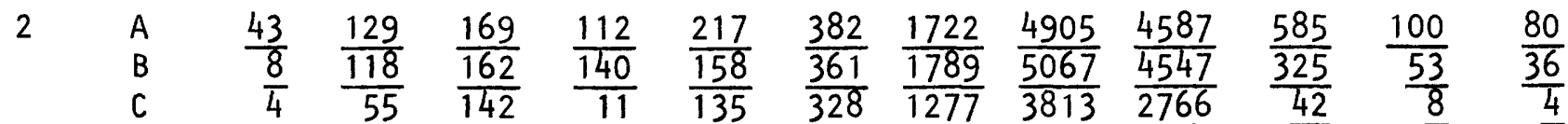

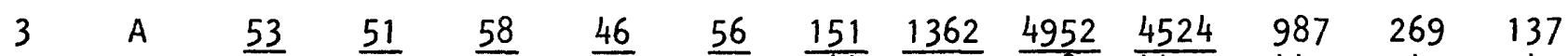

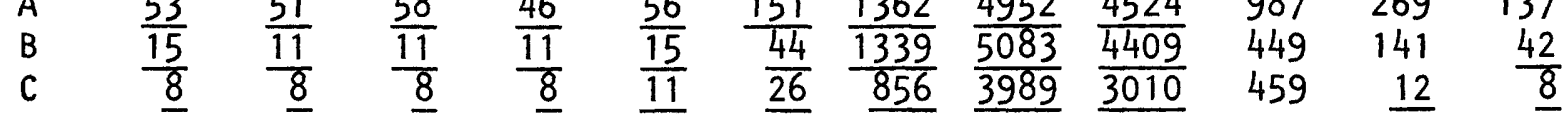

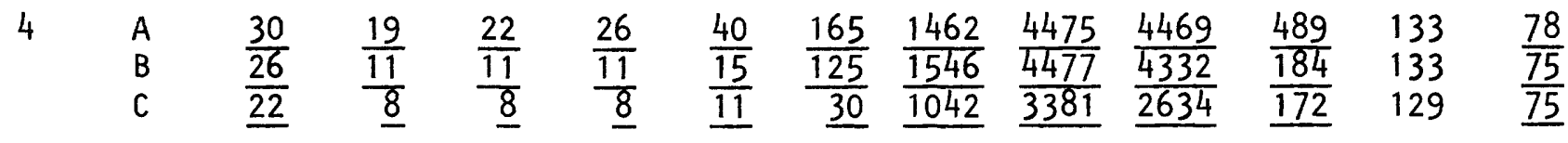


Model-simulated historical monthly streamflows for control point 18 (Yampa River near Maybell, Colo.) are presented in tables 32 through 36 . This site is located downstream from the proposed Juniper Reservoir and approximately $1 \mathrm{mi}$ east of the town of Maybell (fig. 1). In comparison to streamflow-gaging-station measured data, simulated historical mean monthly streamflows for historical (192776) conditions without transmountain diversions (table 32) range from +6 to -23 percent and have an average absolute variation of 7 percent. These simulated historical flow statistics were generally lower than the calculated flow statistics during July, August, and September. All monthly flow values in tables 32 through 36 less than the corresponding historical monthly flow values are underscored.

Table 32.--Summary of monthly streamflows, control point 18 (Yampa River near Maybelz, Colo.), for simulated historical conditions, including 100 percent of transmountain diversions, and for historical conditions

[FLOW VALUES: $A=M E A N ; B=M E D I A N$; and $C=80-P E R C E N T$ EXCEEDENCE. Underscored values are less than historical conditions without transmountain diversions]

FLOW

MONTHLY FLOWS, IN CUBIC FEET PER SECOND

VALUES OCT. NOV. DEC. JAN. FEB. MAR. APR. MAY JUNE JULY AUG. SEPT

\section{SIMULATED HISTORICAL CONDITIONS}

$\begin{array}{rrrrrrrrrrrrr}\text { A } & 352 & 337 & 313 & 290 & 323 & 713 & 2794 & 6228 & 5277 & 1210 & 291 & 200 \\ \text { B } & 323 & 322 & 295 & 277 & 287 & 575 & 2616 & 5962 & 5331 & 1043 & 246 & 158 \\ \text { C } & 219 & 239 & 259 & 245 & 259 & 523 & 1840 & 4376 & 3644 & 459 & 110 & 81\end{array}$

SIMULATED HISTORICAL CONDITIONS WITH 100 PERCENT OF TRANSMOUNTAIN DIVERSIONS

$\begin{array}{lllllllllllll}\text { A } & \frac{273}{236} & \frac{233}{230} & \frac{227}{216} & \frac{212}{199} & \frac{245}{209} & \frac{635}{497} & \frac{2410}{2225} & \frac{5837}{5571} & \frac{4891}{4940} & \frac{955}{743} & \frac{228}{178} & \frac{132}{83} \\ \text { C } & \frac{1346}{146} & \frac{131}{171} & \frac{167}{167} & \underline{182} & \underline{445} & \underline{1449} & \underline{3985} & \underline{3253} & \underline{331} & \frac{172}{22}\end{array}$

HISTORICAL STREAMFLOWS CALCULATED FROM GAGING-STATION RECORDS

$\begin{array}{lllllllllllll}\text { A } & 353 & 351 & 296 & 274 & 323 & 675 & 2647 & 6208 & 5472 & 1331 & 378 & 245 \\ \text { B } & 324 & 324 & 276 & 266 & 299 & 608 & 2755 & 6210 & 5315 & 1200 & 328 & 202 \\ \text { C } & 191 & 248 & 202 & 207 & 247 & 428 & 1544 & 4322 & 3546 & 545 & 197 & 137\end{array}$


Table 33.--Summary of simulated historical monthly streamflows, control point 18 (Yampa River near Maybell, Colo.),

with 25 percent of agricultural and no transmountain diversions, and with 25 percent of both agricultural and transmountain diversions, and including 100 percent of industrial and municipal diversions for all simulations

[FLOW VALUES: $A=M E A N ; B=M E D I A N ;$ and $C=80-P E R C E N T$ EXCEEDENCE. Underscored values are less than corresponding table 32 historical conditions]

\begin{tabular}{|c|c|c|c|c|c|c|c|c|c|c|c|c|c|}
\hline \multirow{2}{*}{$\begin{array}{l}\text { OP- } \\
\text { TION }\end{array}$} & \multirow{2}{*}{$\begin{array}{l}\text { FLOW } \\
\text { VALUES }\end{array}$} & \multicolumn{12}{|c|}{ MONTHLY FLOWS, IN CUBIC FEET PER SECOND } \\
\hline & & OCT. & NOV. & DEC. & JAN. & FEB. & MAR. & APR. & MAY & JUNE & JULY & $A \cup G$. & SEPT \\
\hline \multicolumn{14}{|c|}{ WITHOUT TRANSMOUNTAIN DIVERSIONS } \\
\hline 1 & $\begin{array}{l}A \\
B \\
C\end{array}$ & $\begin{array}{l}466 \\
505 \\
398\end{array}$ & $\begin{array}{l}551 \\
578 \\
496\end{array}$ & $\begin{array}{l}575 \\
596 \\
544\end{array}$ & $\begin{array}{l}593 \\
606 \\
551\end{array}$ & $\begin{array}{l}548 \\
575 \\
517\end{array}$ & $\frac{\frac{397}{418}}{263}$ & $\frac{\frac{517}{200}}{200}$ & $\frac{\frac{3034}{2533}}{723}$ & $\frac{\frac{3406}{3165}}{1586}$ & $\frac{\frac{886}{805}}{696}$ & $\frac{\frac{208}{200}}{200}$ & $\begin{array}{l}309 \\
326 \\
262\end{array}$ \\
\hline 2 & $\begin{array}{l}A \\
B \\
C\end{array}$ & $\begin{array}{l}485 \\
516 \\
414\end{array}$ & $\begin{array}{l}573 \\
595 \\
523\end{array}$ & $\begin{array}{l}594 \\
610 \\
566\end{array}$ & $\begin{array}{l}586 \\
603 \\
547\end{array}$ & $\begin{array}{l}556 \\
585 \\
503\end{array}$ & $\frac{\frac{397}{423}}{266}$ & $\frac{\frac{525}{200}}{200}$ & $\frac{\frac{3433}{3151}}{753}$ & $\frac{\frac{3891}{3615}}{1930}$ & $\frac{\frac{902}{804}}{714}$ & $\frac{\frac{204}{200}}{200}$ & $\begin{array}{l}306 \\
323 \\
262\end{array}$ \\
\hline 3 & $\begin{array}{l}A \\
B \\
C\end{array}$ & $\begin{array}{l}485 \\
516 \\
414\end{array}$ & $\begin{array}{l}573 \\
595 \\
523\end{array}$ & $\begin{array}{l}594 \\
610 \\
566\end{array}$ & $\begin{array}{l}586 \\
603 \\
547\end{array}$ & $\begin{array}{l}556 \\
585 \\
503\end{array}$ & $\frac{\frac{382}{418}}{243}$ & $\frac{\frac{588}{200}}{200}$ & $\frac{\frac{3191}{2919}}{895}$ & $\frac{\frac{3694}{3350}}{1753}$ & $\frac{\frac{906}{813}}{721}$ & $\frac{\frac{203}{200}}{200}$ & $\begin{array}{l}309 \\
327 \\
260\end{array}$ \\
\hline 4 & $\begin{array}{l}A \\
B \\
C\end{array}$ & $\frac{\frac{250}{205}}{147}$ & $\frac{\frac{196}{180}}{97}$ & $\frac{\frac{213}{207}}{160}$ & $\frac{\frac{217}{209}}{170}$ & $\frac{\frac{255}{222}}{191}$ & $\frac{562}{\frac{536}{396}}$ & $\frac{\frac{2353}{2217}}{1539}$ & $\frac{\frac{5728}{5473}}{\underline{3959}}$ & $\frac{\frac{5137}{5204}}{3547}$ & $\frac{1132}{\frac{953}{379}}$ & $\frac{\frac{241}{200}}{105}$ & $\frac{\frac{143}{102}}{44}$ \\
\hline \multicolumn{14}{|c|}{ WITH TRANSMOUNTAIN DIVERSIONS } \\
\hline 1 & $\begin{array}{l}A \\
B \\
C\end{array}$ & $\begin{array}{l}455 \\
497 \\
360\end{array}$ & $\begin{array}{l}546 \\
571 \\
495\end{array}$ & $\begin{array}{l}576 \\
599 \\
558\end{array}$ & $\begin{array}{l}590 \\
601 \\
551\end{array}$ & $\begin{array}{l}544 \\
571 \\
500\end{array}$ & $\frac{\frac{384}{417}}{265}$ & $\frac{\frac{458}{200}}{200}$ & $\frac{\frac{2749}{1872}}{\underline{717}}$ & $\frac{\frac{3260}{3067}}{1179}$ & $\frac{\frac{872}{796}}{686}$ & $\frac{\frac{208}{200}}{200}$ & $\begin{array}{l}306 \\
323 \\
245\end{array}$ \\
\hline 2 & $\begin{array}{l}A \\
B \\
C\end{array}$ & $\begin{array}{l}470 \\
510 \\
382\end{array}$ & $\begin{array}{l}549 \\
579 \\
508\end{array}$ & $\begin{array}{l}577 \\
595 \\
552\end{array}$ & $\begin{array}{l}586 \\
592 \\
546\end{array}$ & $\begin{array}{l}550 \\
566 \\
504\end{array}$ & $\frac{\frac{394}{424}}{267}$ & $\frac{\frac{457}{200}}{200}$ & $\frac{\frac{3153}{2603}}{749}$ & $\frac{\frac{3761}{3511}}{1733}$ & $\frac{888}{\frac{803}{691}}$ & $\frac{\frac{205}{200}}{200}$ & $\begin{array}{l}305 \\
322 \\
257\end{array}$ \\
\hline 3 & $\begin{array}{l}A \\
B \\
C\end{array}$ & $\begin{array}{l}481 \\
516 \\
388\end{array}$ & $\begin{array}{l}565 \\
593 \\
516\end{array}$ & $\begin{array}{l}586 \\
607 \\
554\end{array}$ & $\begin{array}{l}582 \\
601 \\
546\end{array}$ & $\begin{array}{l}557 \\
582 \\
516\end{array}$ & $\frac{\frac{382}{414}}{246}$ & $\frac{\frac{508}{200}}{200}$ & $\frac{\frac{2902}{2214}}{727}$ & $\frac{\frac{3565}{3245}}{1537}$ & $\frac{\frac{888}{809}}{685}$ & $\frac{\frac{204}{200}}{200}$ & $\begin{array}{l}308 \\
327 \\
258\end{array}$ \\
\hline 4 & $\begin{array}{l}A \\
B \\
C\end{array}$ & $\frac{\frac{243}{200}}{145}$ & $\frac{\frac{154}{130}}{\underline{31}}$ & $\frac{\frac{170}{188}}{49}$ & $\frac{\frac{194}{190}}{139}$ & $\frac{\frac{232}{196}}{171}$ & $\frac{\frac{542}{416}}{376}$ & $\frac{\frac{2255}{2119}}{1442}$ & $\frac{\frac{5630}{5375}}{\underline{3861}}$ & $\frac{\frac{5039}{5106}}{\frac{3449}{4}}$ & $\frac{1058}{\frac{856}{365}}$ & $\frac{\frac{232}{190}}{103}$ & $\frac{130}{\frac{79}{33}}$ \\
\hline
\end{tabular}


Table 34.--Summary of simulated historical monthly streamflows, control point 18 (Yampa River near Maybell, Colo.),

with 50 percent of agricultural and no transmountain diversions, and with 50 percent of both agricultural and transmountain diversions, and including 100 percent of industrial and municipal diversions for all simulations

[FLOW VALUES: $A=M E A N ; B=M E D I A N$; and $C=80-P E R C E N T$ EXCEEDENCE. Underscored values are less than corresponding table 32 historical conditions]

WITHOUT TRANSMOUNTAIN DIVERSIONS

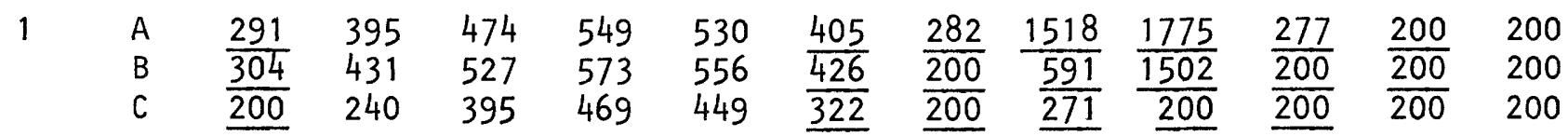

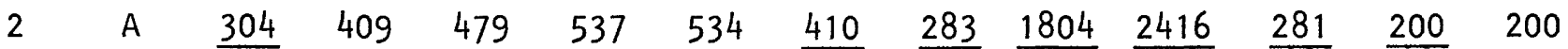

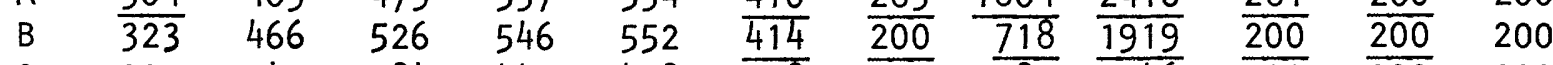

$\begin{array}{lllllllllllll}\text { C } & 200 & 245 & 384 & 447 & 418 & \underline{338} & \underline{200} & \underline{787} & \underline{246} & \underline{200} & \overline{200} & 200\end{array}$

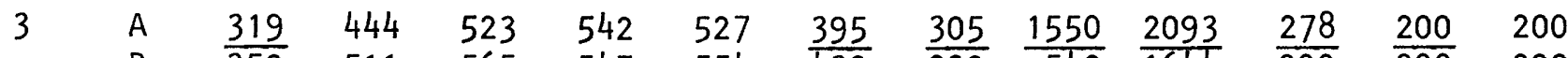

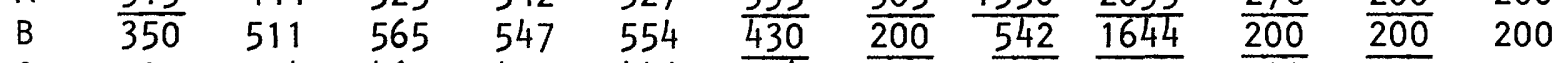

$\begin{array}{lllllllllllll}\text { C } & 200 & 254 & 467 & 477 & 446 & \underline{294} & \underline{200} & \underline{200} & \underline{232} & \underline{200} & \frac{200}{200} & 200\end{array}$

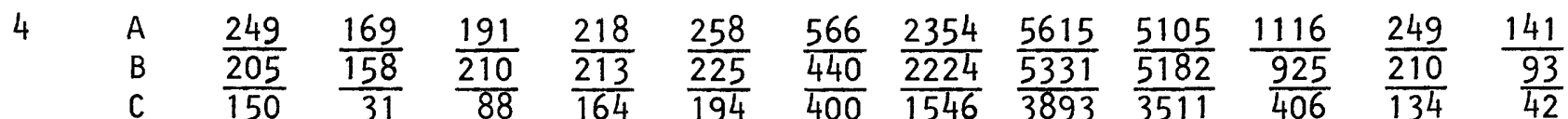

WITH TRANSMOUNTAIN DIVERSIONS

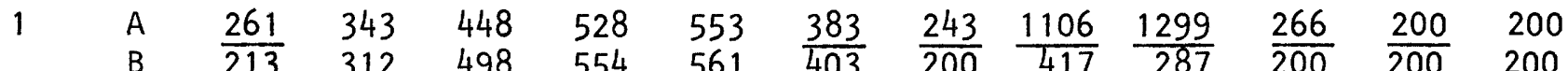

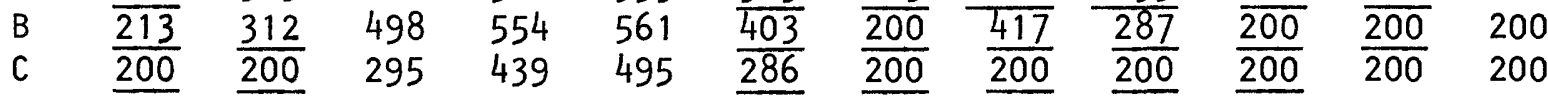

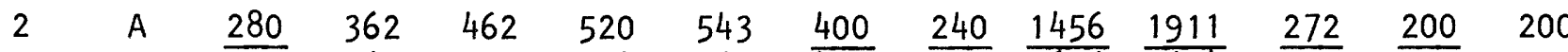

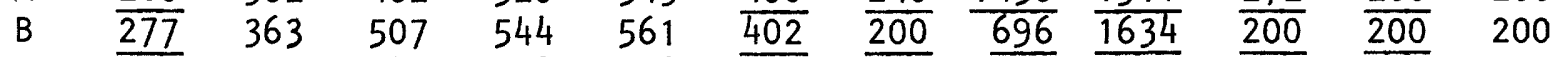

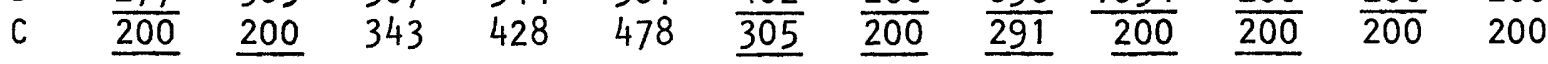

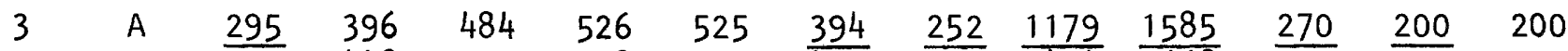

$\begin{array}{lllllllllll}B & \frac{29}{322} & 448 & 535 & 558 & 559 & \overline{427} & \frac{200}{200} & \frac{424}{668} & \frac{200}{200} & \frac{200}{200}\end{array}$

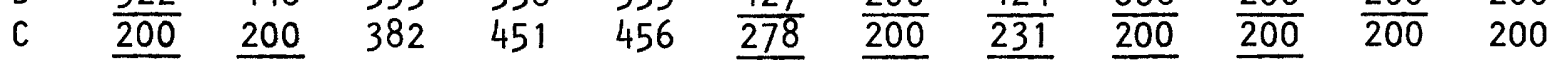

4 A $\quad \frac{235}{196} \quad \frac{120}{78} \quad \frac{112}{85} \quad \frac{165}{169} \quad \frac{173}{167} \quad \frac{484}{384} \quad \frac{2159}{2028} \quad \frac{5419}{5139} \quad \frac{4909}{4986} \quad \frac{996}{760} \quad \frac{242}{208} \quad \frac{123}{73}$

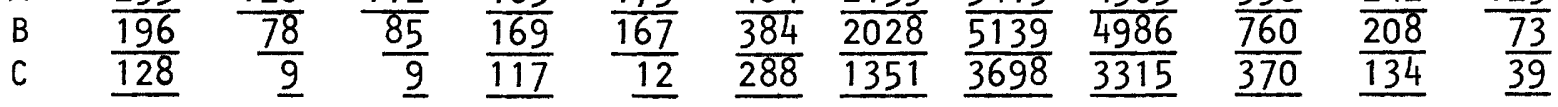


Table 35.--Summary of simulated historical monthly streamflows, control point 18 (Yampa River near Maybell, Colo.),

with 75 percent of agricultural and no transmountain diversions, and with 75 percent of both agricultural and transmountain diversions, and including 100 percent of industrial and municipal diversions for all simulations

[FLOW VALUES: $A=M E A N ; B=M E D I A N ;$ and $C=80-P E R C E N T$ EXCEEDENCE. Underscored values are less than corresponding table 32 historical conditions]

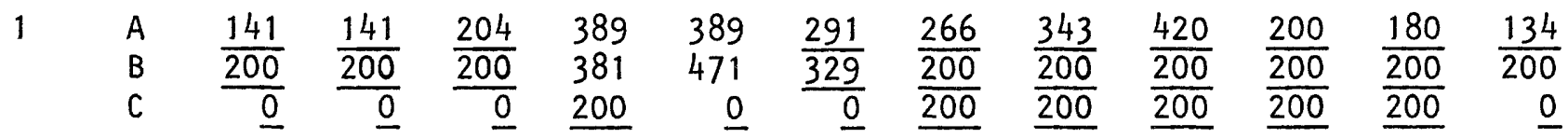

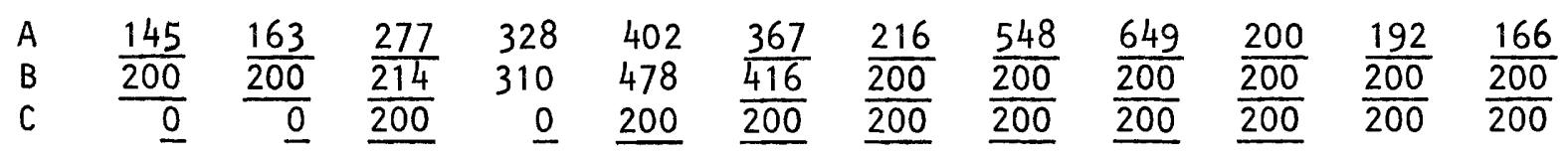

$$
\begin{array}{lllllllllllll}
\text { A } & \frac{145}{200} & \frac{160}{200} & \frac{220}{200} & \frac{281}{200} & \frac{318}{325} & \frac{258}{280} & \frac{189}{200} & \frac{478}{200} & \frac{510}{200} & \frac{200}{200} & \frac{192}{200} & \frac{173}{200} \\
\text { C } & \frac{\underline{0}}{\underline{0}} & \frac{\underline{0}}{\underline{0}} & \frac{\underline{0}}{0} & \underline{0} & \underline{0} & \underline{0} & \underline{200} & \underline{200} & \underline{200} & \frac{\underline{0}}{200} & 200
\end{array}
$$

\begin{tabular}{|c|c|c|c|c|c|c|c|c|c|c|c|}
\hline$\frac{\frac{243}{204}}{130}$ & $\frac{135}{\frac{78}{9}}$ & $\frac{\frac{154}{152}}{9}$ & $\frac{\frac{207}{205}}{162}$ & $\frac{\frac{239}{219}}{185}$ & $\frac{\frac{568}{444}}{400}$ & $\frac{\frac{2355}{2227}}{1549}$ & $\frac{5570}{\frac{5272}{3837}}$ & $\frac{\frac{5077}{5151}}{\frac{5489}{4}}$ & $\frac{1101}{\frac{898}{446}}$ & $\frac{\frac{271}{233}}{166}$ & $\frac{139}{91}$ \\
\hline
\end{tabular}

WITH TRANSMOUNTAIN DIVERSIONS

$$
\begin{array}{lllllllllllll}
\text { A } & \frac{100}{100} & \frac{110}{0} & \frac{161}{200} & \frac{186}{100} & \frac{270}{220} & \frac{274}{297} & \frac{262}{200} & \frac{264}{200} & \frac{289}{200} & \frac{187}{200} & \frac{136}{200} & \frac{114}{200} \\
\text { C } & \frac{\underline{0}}{\underline{0}} & \underline{0} & \frac{\underline{0}}{\underline{0}} & \frac{\underline{200}}{200} & \underline{200} & \underline{200} & \frac{\underline{0}}{\underline{0}} & \frac{\underline{0}}{\underline{0}}
\end{array}
$$

$$
\begin{array}{lllllllllllll}
\text { A } & \frac{132}{200} & \frac{160}{200} & \frac{217}{200} & \frac{283}{200} & \frac{320}{307} & \frac{266}{271} & \frac{232}{200} & \frac{384}{200} & \frac{439}{200} & \frac{192}{200} & \frac{173}{200} & \frac{132}{200} \\
\text { C } & \frac{10}{0} & \frac{10}{0} & \frac{\underline{0}}{0} & \frac{\underline{0}}{200} & \underline{200} & \underline{200} & \underline{200} & \frac{1}{200} & \underline{0}
\end{array}
$$

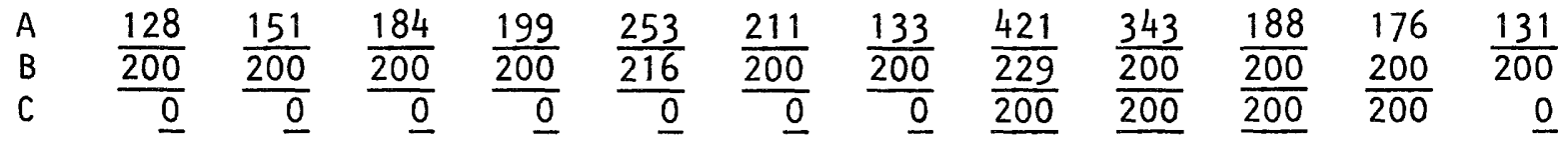

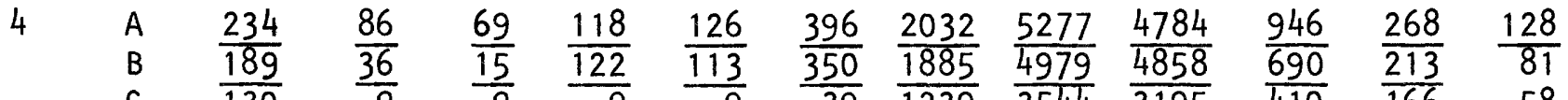

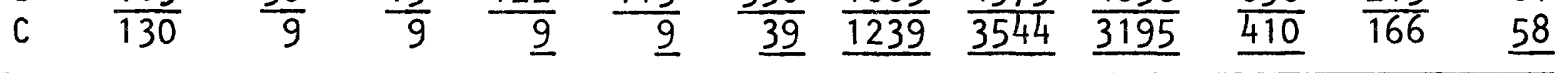


Table 36.--Summary of simulated historical monthly streamflows, control point 18 (Yampa River near Maybell, Colo.),

with 100 percent of agricultural and no transmountain diversions, and with 100 percent of both agricultural and transmountain diversions, and including 100 percent of industrial and municipal diversions for all simulations

[FLOW VALUES: $A=M E A N ; B=M E D I A N ;$ and $C=80-P E R C E N T$ EXCEEDENCE. Underscored values are less than corresponding table 32 historical conditions]

OP- FLOW

MONTHLY FLOWS, IN CUBIC FEET PER SECOND

TION VALUES

OCT. NOV. DEC. JAN. FEB. MAR. APR. MAY JUNE JULY AUG. SEPT

WITHOUT TRANSMOUNTAIN DIVERSIONS

$\begin{array}{llllllllllllll}\text { A A } & \underline{48} & \frac{108}{0} & \frac{124}{0} & \frac{272}{200} & \frac{348}{440} & \frac{189}{0} & \frac{268}{200} & \frac{200}{200} & \frac{210}{200} & \frac{164}{200} & \frac{75}{0} & \frac{53}{0} \\ \text { B } & \underline{0} & \underline{0} & \underline{0} & \frac{0}{0} & \frac{\underline{0}}{0} & \underline{0} & \underline{200} & \underline{200} & \underline{200} & \underline{200} & \underline{0} & \underline{0}\end{array}$

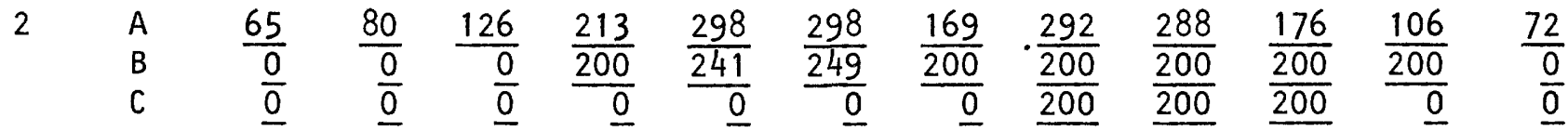

$\begin{array}{llllllllllllll}\mathrm{A} & \underline{60} & \frac{82}{0} & \underline{78} & \frac{103}{0} & \frac{144}{0} & \frac{162}{0} & \frac{113}{200} & \frac{275}{200} & \frac{281}{200} & \frac{180}{200} & \frac{110}{200} & \frac{72}{0} \\ \mathrm{~B} & \underline{0} & \underline{0} & \underline{0} & \underline{0} & \underline{0} & \underline{0} & \frac{0}{0} & \frac{200}{200} & \underline{200} & \underline{200} & \underline{0} & \underline{0}\end{array}$

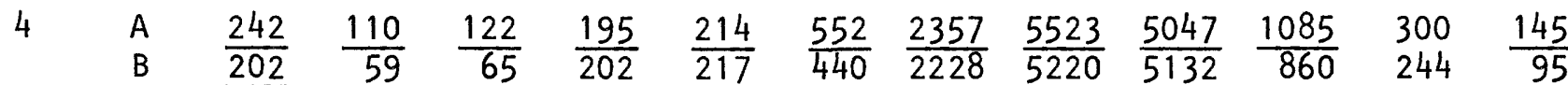

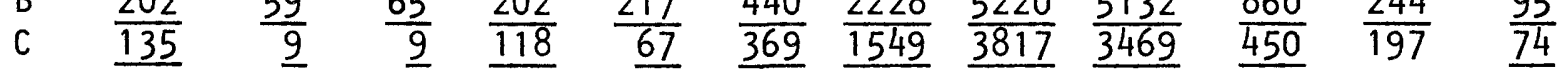

WITH TRANSMOUNTAIN DIVERSIONS

$\begin{array}{llllllllllllll}\mathrm{A} & \underline{31} & \underline{33} & \underline{63} & \frac{143}{0} & \frac{123}{0} & \underline{181} & \frac{283}{200} & \frac{200}{200} & \frac{192}{200} & \frac{108}{200} & \frac{60}{0} & \frac{28}{0} \\ \mathrm{~B} & \underline{0} & \underline{0} & \frac{0}{0} & \underline{0} & \underline{0} & \underline{0} & \frac{200}{200} & \frac{200}{200} & \underline{200} & \underline{0} & \underline{0} & \underline{0}\end{array}$

$\begin{array}{llllllllllllll}\text { A } & \frac{52}{0} & \frac{90}{0} & \frac{76}{0} & \frac{124}{0} & \frac{228}{0} & \frac{299}{250} & \frac{167}{200} & \frac{229}{200} & \frac{264}{200} & \frac{145}{200} & \frac{73}{0} & \frac{48}{0} \\ \text { B } & \frac{0}{0} & \frac{0}{0} & \frac{0}{0} & \frac{0}{0} & \frac{0}{0} & \frac{150}{0} & \frac{10}{0}\end{array}$

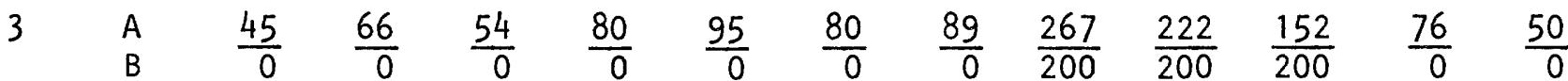

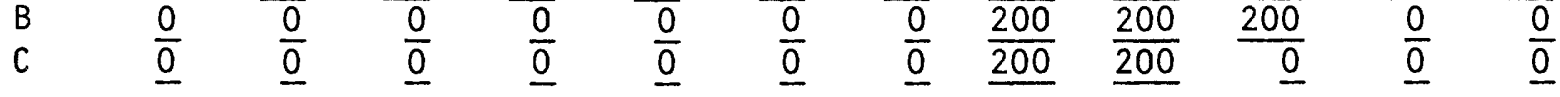

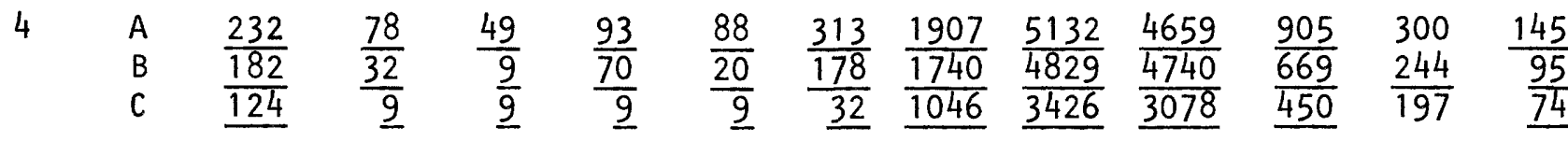


Juniper Reservoir, a large agricultural diversion, is located upstream from the Yampa River at Maybell location. The assumed agricultural diversion simulated for Juniper Reservoir would divert as much as eight times the amount of water as the upstream Vidler tunnel transmountain diversion. Yampa River near Maybell (control point 18) is also the streamflow-gaging site and index station for streamflow from the Yampa River for the Colorado River Compact of 1948. This agreement requires that $5,000,000$ acre-ft of water per 10-year period be delivered from the Yampa River basin upstream, which represents an approximate continuous flow of $690 \mathrm{ft}^{3} / \mathrm{s}$.

For the Yampa River near Maybell site, the Juniper Reservoir is included for reservoir-development options 1,2 , and 3 (table 2) and results in more regulated flows than option 4 (tables 33 through 36). Reservoir-development option 4 without Juniper Reservoir could follow a reduced annual-flow pattern similar to the historical flows (tables 32 and 33 ).

For reservoir-development options 1,2, and 3, simulated historical 50-year median monthly flows quickly drop to the desired flow of $200 \mathrm{ft}^{3} / \mathrm{s}$. The 80 -percent exceedence value dropped to zero for 3 or more months at the 75 -percent allocation percentage (table 35). Realistically, if any substantial flow is to be maintained in the Yampa River at this location, reservoir-development options 1, 2, and 3 would be restricted to the 50-percent agricultural water-use allocation or less.

At the 100-percent allocation level, the Vidler transmountain diversion alone could cause an approximate 12 -percent reduction in flow, as seen in table 32 . When combined with the large irrigation diversion from this site (options 1, 2, and 3 ), the Vidler transmountain diversion could increase the number of zero-flow occurrences as the percentage of water-use allocation increases and could reduce the possibility of maintaining a desired flow of $200 \mathrm{ft}^{3} / \mathrm{s}$ (tables 33 through 36 ).

Model-simulated historical monthly streamflows for control point 19 (Yampa River near Lily, Colo.) are presented in tables 37 through 41 . This site is located downstream from the proposed Juniper and Cross Mountain Reservoirs (fig. 1) and is approximately $2 \mathrm{mi}$ upstream from the confluence of the Little Snake River. All monthly flow statistics in tables 37 through 41 less than the corresponding historical-flow statistics are underscored. 
Table 37.--Summary of simulated historical monthly streamflows, control point 19 (Yampa River near Lily, Colo.L),

for historical conditions and with 100 percent of transmountain diversions

[FLOW VALUES: $A=M E A N ; B=M E D I A N ;$ and $C=80-P E R C E N T$ EXCEEDENCE. Underscored values are less than historical conditions without transmountain diversions]

FLOW

MONTHLY FLOWS, IN CUBIC FEET PER SECOND

VALUES OCT. NOV. DEC. JAN. FEB. MAR. APR. MAY JUNE JULY AUG. SEPT

SIMULATED HISTORICAL CONDITIONS

$\begin{array}{rrrrrrrrrrrrr}\text { A } & 411 & 398 & 364 & 337 & 379 & 831 & 3260 & 7311 & 6220 & 1440 & 357 & 240 \\ \text { B } & 377 & 374 & 337 & 320 & 332 & 666 & 3033 & 7037 & 6305 & 1247 & 304 & 195 \\ \text { C } & 253 & 283 & 294 & 288 & 304 & 605 & 2060 & 5137 & 4315 & 556 & 142 & 104\end{array}$

SIMULATED HISTORICAL CONDITIONS WITH 100 PERCENT OF TRANSMOUNTAIN DIVERSIONS

\begin{tabular}{|c|c|c|c|c|c|c|c|c|c|c|}
\hline$\frac{\frac{332}{292}}{177}$ & $\frac{\frac{293}{283}}{117}$ & $\frac{\frac{278}{257}}{211}$ & $\frac{259}{\frac{242}{204}}$ & $\frac{301}{\frac{254}{226}}$ & $\frac{\frac{753}{588}}{527}$ & $\frac{2876}{\frac{2642}{1669}}$ & $\frac{\frac{6920}{6646}}{4746}$ & $\frac{\frac{5833}{5914}}{\frac{3924}{4}}$ & $\frac{1185}{\frac{951}{401}}$ & $\frac{293}{\frac{235}{111}}$ \\
\hline
\end{tabular}


Table 38.--Summary of simulated historical monthly streamflows, control point 19 (Yampa River near Lily, Colo.),

with 25 percent of agricultural and no transmountain diversions, and with 25 percent of both agricultural and transmountain diversions, and including 100 percent of industrial and municipal diversions for all simulations

[FLOW VALUES: $A=M E A N ; B=M E D I A N ;$ and $C=80$-PERCENT EXCEEDENCE. Underscored values are less than corresponding table 37 simulated historical conditions]

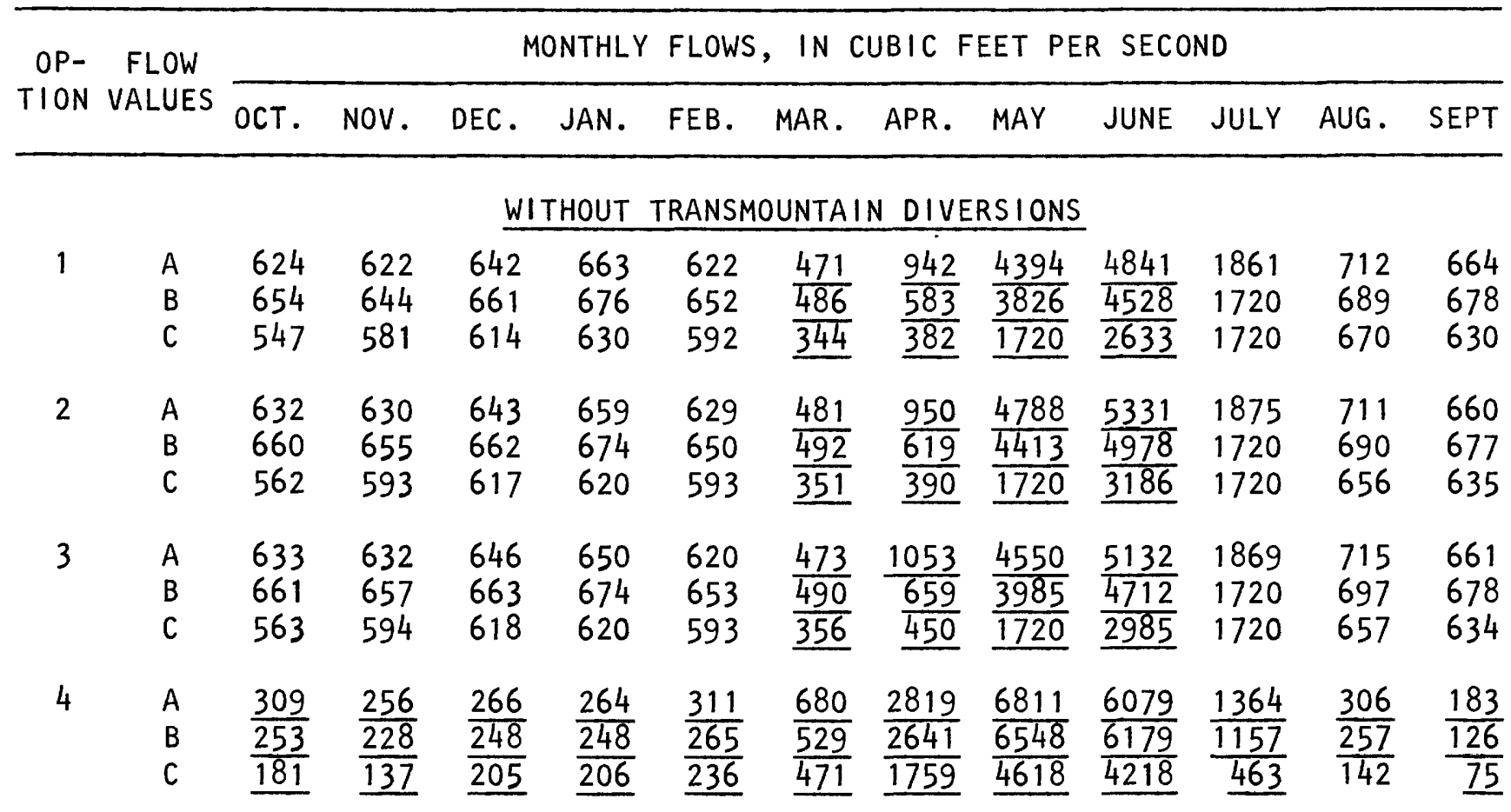

WITH TRANSMOUNTAIN DIVERSIONS

\begin{tabular}{|c|c|c|c|c|c|c|c|c|c|c|c|c|c|}
\hline 1 & $\begin{array}{l}A \\
B \\
C\end{array}$ & $\begin{array}{l}624 \\
654 \\
547\end{array}$ & $\begin{array}{l}622 \\
644 \\
581\end{array}$ & $\begin{array}{l}642 \\
661 \\
614\end{array}$ & $\begin{array}{l}663 \\
676 \\
630\end{array}$ & $\begin{array}{l}622 \\
652 \\
592\end{array}$ & $\frac{\frac{463}{477}}{344}$ & $\frac{\frac{859}{530}}{367}$ & $\frac{\frac{4105}{3314}}{1720}$ & $\frac{4693}{\frac{4430}{2522}}$ & $\begin{array}{l}1851 \\
1720 \\
1720\end{array}$ & $\begin{array}{l}709 \\
688 \\
670\end{array}$ & $\begin{array}{l}664 \\
678 \\
630\end{array}$ \\
\hline 2 & $\begin{array}{l}A \\
B \\
C\end{array}$ & $\begin{array}{l}631 \\
660 \\
562\end{array}$ & $\begin{array}{l}627 \\
646 \\
592\end{array}$ & $\begin{array}{l}643 \\
661 \\
616\end{array}$ & $\begin{array}{l}660 \\
673 \\
620\end{array}$ & $\begin{array}{l}628 \\
648 \\
597\end{array}$ & $\frac{\frac{481}{493}}{348}$ & $\frac{\frac{860}{564}}{\underline{368}}$ & $\frac{\frac{4506}{3875}}{1720}$ & $\frac{\frac{5199}{4875}}{2779}$ & $\begin{array}{l}1862 \\
1720 \\
1720\end{array}$ & $\begin{array}{l}708 \\
689 \\
656\end{array}$ & $\begin{array}{l}660 \\
677 \\
632\end{array}$ \\
\hline 3 & $\begin{array}{l}A \\
B \\
C\end{array}$ & $\begin{array}{l}633 \\
661 \\
563\end{array}$ & $\begin{array}{l}630 \\
652 \\
594\end{array}$ & $\begin{array}{l}644 \\
663 \\
618\end{array}$ & $\begin{array}{l}649 \\
674 \\
620\end{array}$ & $\begin{array}{l}620 \\
651 \\
593\end{array}$ & $\frac{\frac{413}{490}}{351}$ & $\frac{953}{\frac{927}{443}}$ & $\frac{\frac{4263}{3641}}{1720}$ & $\frac{4999}{4608}$ & $\begin{array}{l}1857 \\
1720 \\
1720\end{array}$ & $\begin{array}{l}712 \\
696 \\
657\end{array}$ & $\begin{array}{l}678 \\
633\end{array}$ \\
\hline 4 & $\begin{array}{l}A \\
B \\
C\end{array}$ & $\frac{\frac{302}{247}}{181}$ & $\frac{\frac{215}{184}}{75}$ & $\frac{\frac{223}{229}}{90}$ & $\frac{\frac{241}{228}}{181}$ & $\frac{\frac{288}{243}}{216}$ & $\frac{\frac{660}{510}}{\frac{452}{4}}$ & $\frac{\frac{2721}{2544}}{1662}$ & $\frac{\frac{6713}{6450}}{4521}$ & $\frac{\frac{5982}{6081}}{\frac{4120}{}}$ & $\frac{\frac{1290}{1059}}{442}$ & $\frac{\frac{297}{250}}{142}$ & $\frac{170}{\frac{107}{57}}$ \\
\hline
\end{tabular}


Table 39.--Summary of simulated historical monthly streamflows, control point 19 (Yampa River near Lily, Colo.),

with 50 percent of agricultural and no transmountain diversions, and with 50 percent of both agricultural and transmountain diversions, and including 100 percent of industrial and municipal diversions for all simulations

[FLOW VALUES: $A=M E A N ; B=M E D I A N ;$ and $C=80-P E R C E N T$ EXCEEDENCE. Underscored values are less than corresponding table 37 simulated historical conditions]

\begin{tabular}{|c|c|c|c|c|c|c|c|c|c|c|c|c|c|}
\hline \multirow{2}{*}{$\begin{array}{l}\text { OP- } \\
\text { TION }\end{array}$} & \multirow{2}{*}{$\begin{array}{l}\text { FLOW } \\
\text { VALUES }\end{array}$} & \multicolumn{12}{|c|}{ MONTHLY FLOWS, IN CUBIC FEET PER SECOND } \\
\hline & & OCT. & NOV. & DEC. & JAN. & FEB. & MAR. & APR. & MAY & JUNE & JULY & AUG. & SEPT \\
\hline
\end{tabular}

WITHOUT TRANSMOUNTAIN DIVERSIONS

\begin{tabular}{|c|c|c|c|c|c|c|c|c|c|c|c|c|c|}
\hline 1 & $\begin{array}{l}A \\
B \\
C\end{array}$ & $\begin{array}{l}615 \\
644 \\
537\end{array}$ & $\begin{array}{l}622 \\
644 \\
581\end{array}$ & $\begin{array}{l}642 \\
661 \\
614\end{array}$ & $\begin{array}{l}663 \\
676 \\
630\end{array}$ & $\begin{array}{l}622 \\
652 \\
592\end{array}$ & $\frac{\frac{468}{477}}{344}$ & $\frac{\frac{565}{344}}{\underline{344}}$ & $\frac{\frac{3061}{1720}}{1720}$ & $\frac{\frac{3721}{3262}}{1798}$ & $\begin{array}{l}1881 \\
1754 \\
1720\end{array}$ & $\begin{array}{l}1252 \\
1275 \\
1230\end{array}$ & $\begin{array}{l}834 \\
830 \\
809\end{array}$ \\
\hline & $\begin{array}{l}A \\
B \\
C\end{array}$ & $\begin{array}{l}622 \\
652 \\
561\end{array}$ & $\begin{array}{l}622 \\
644 \\
596\end{array}$ & $\begin{array}{l}631 \\
650 \\
606\end{array}$ & $\begin{array}{l}650 \\
650 \\
607\end{array}$ & $\begin{array}{l}624 \\
637 \\
586\end{array}$ & $\frac{\frac{475}{486}}{344}$ & $\frac{\frac{589}{415}}{344}$ & $\frac{\frac{3344}{1737}}{1720}$ & $\frac{\frac{4374}{3698}}{1993}$ & $\begin{array}{l}1884 \\
1754 \\
1720\end{array}$ & $\begin{array}{l}1256 \\
1275 \\
1235\end{array}$ & $\begin{array}{l}834 \\
830 \\
809\end{array}$ \\
\hline & $\begin{array}{l}A \\
B \\
C\end{array}$ & $\begin{array}{l}623 \\
652 \\
563\end{array}$ & $\begin{array}{l}626 \\
639 \\
598\end{array}$ & $\begin{array}{l}638 \\
659 \\
609\end{array}$ & $\begin{array}{l}645 \\
665 \\
624\end{array}$ & $\begin{array}{l}617 \\
640 \\
591\end{array}$ & $\frac{\frac{470}{481}}{344}$ & $\frac{\frac{643}{491}}{344}$ & $\frac{\frac{3136}{1819}}{1720}$ & $\frac{\frac{4049}{3439}}{1832}$ & $\begin{array}{l}1882 \\
1753 \\
1720\end{array}$ & $\begin{array}{l}1259 \\
1275 \\
1235\end{array}$ & $\begin{array}{l}834 \\
829 \\
808\end{array}$ \\
\hline & $\begin{array}{l}A \\
B \\
C\end{array}$ & $\frac{\frac{308}{253}}{186}$ & $\frac{229}{\frac{208}{75}}$ & $\frac{\frac{244}{252}}{123}$ & $\frac{265}{251}$ & $\frac{\frac{314}{269}}{239}$ & $\frac{\frac{684}{533}}{475}$ & $\frac{\frac{2820}{2646}}{1766}$ & $\frac{\frac{6698}{6396}}{4547}$ & $\frac{\frac{6047}{6156}}{4182}$ & $\frac{\frac{1348}{1128}}{500}$ & $\frac{\frac{314}{271}}{173}$ & $\frac{182}{\frac{119}{70}}$ \\
\hline
\end{tabular}

WITH TRANSMOUNTAIN DIVERSIONS

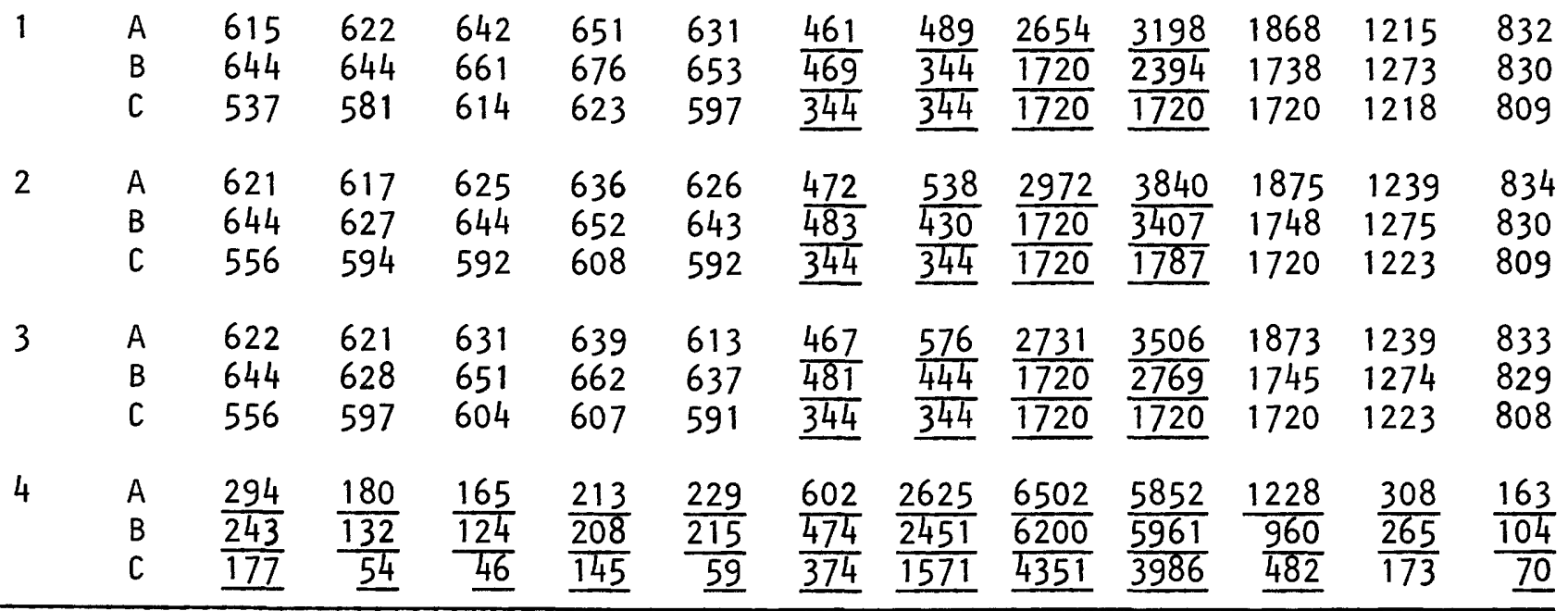


Table 40.--Summary of simulated historical monthly streamflows, control point 19 (Yampa River near Lily, Colo.),

with 75 percent of agricultural and no transmountain diversions, and with 75 percent of both agricultural and transmountain diversions, and including 100 percent of industrial and municipal diversions for all simulations

[FLOW VALUES: $A=M E A N ; B=M E D I A N ;$ and $C=80$-PERCENT EXCEEDENCE. Underscored values are less than corresponding table 37 simulated historical conditions]

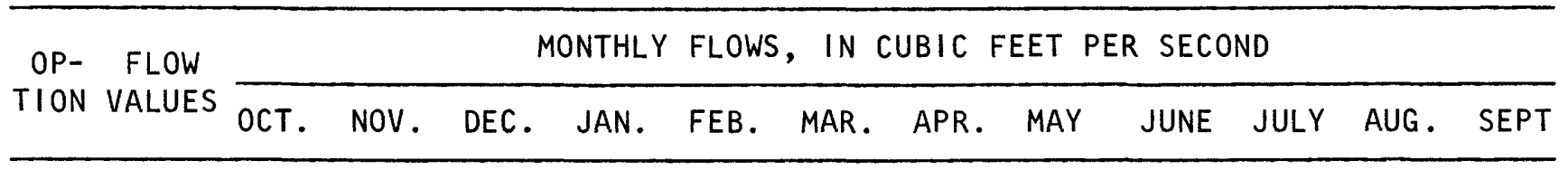

WITHOUT TRANSMOUNTAIN DIVERSIONS

\begin{tabular}{|c|c|c|c|c|c|c|c|c|c|c|c|c|c|}
\hline & $\begin{array}{l}A \\
B \\
C\end{array}$ & $\begin{array}{l}606 \\
634 \\
527\end{array}$ & $\begin{array}{l}608 \\
613 \\
580\end{array}$ & $\begin{array}{l}539 \\
638 \\
501\end{array}$ & $\begin{array}{l}574 \\
655 \\
568\end{array}$ & $\begin{array}{l}514 \\
617 \\
188\end{array}$ & $\frac{\frac{381}{385}}{206}$ & $\frac{\frac{372}{344}}{344}$ & $\frac{\frac{1986}{1720}}{1720}$ & $\frac{\frac{2538}{2270}}{1720}$ & $\begin{array}{l}2398 \\
2410 \\
2256\end{array}$ & $\begin{array}{l}1707 \\
1792 \\
1760\end{array}$ & $\begin{array}{r}972 \\
1128 \\
613\end{array}$ \\
\hline$?$ & $\begin{array}{l}A \\
B \\
C\end{array}$ & $\begin{array}{l}613 \\
631 \\
560\end{array}$ & $\begin{array}{l}595 \\
605 \\
563\end{array}$ & $\begin{array}{l}579 \\
595 \\
536\end{array}$ & $\begin{array}{l}568 \\
596 \\
522\end{array}$ & $\begin{array}{l}522 \\
596 \\
522\end{array}$ & $\frac{\frac{433}{460}}{344}$ & $\frac{\frac{381}{344}}{344}$ & $\frac{\frac{2197}{1720}}{1720}$ & $\frac{\frac{2820}{2434}}{1720}$ & $\begin{array}{l}2418 \\
2416 \\
2284\end{array}$ & $\begin{array}{l}1773 \\
1792 \\
1761\end{array}$ & $\begin{array}{l}1060 \\
1133 \\
1106\end{array}$ \\
\hline J & $\begin{array}{l}A \\
B \\
C\end{array}$ & $\begin{array}{l}614 \\
631 \\
560\end{array}$ & $\begin{array}{l}598 \\
602 \\
562\end{array}$ & $\begin{array}{l}564 \\
575 \\
517\end{array}$ & $\begin{array}{l}529 \\
584 \\
398\end{array}$ & $\begin{array}{r}450 \\
562 \\
57 \\
\end{array}$ & $\frac{\frac{349}{345}}{88}$ & $\frac{\frac{368}{344}}{344}$ & $\frac{\frac{2070}{1720}}{1720}$ & $\frac{\frac{2635}{2371}}{1720}$ & $\begin{array}{l}2396 \\
2416 \\
2230\end{array}$ & $\begin{array}{l}1782 \\
1792 \\
1761\end{array}$ & $\begin{array}{l}1084 \\
1132 \\
1105\end{array}$ \\
\hline & $\begin{array}{l}A \\
B \\
C\end{array}$ & $\frac{\frac{302}{247}}{170}$ & $\frac{\frac{196}{132}}{57}$ & $\frac{\frac{208}{194}}{48}$ & $\frac{\frac{254}{249}}{197}$ & $\frac{295}{266}$ & $\frac{\frac{686}{536}}{476}$ & $\frac{\frac{2821}{2650}}{1769}$ & $\frac{\frac{6653}{6348}}{4519}$ & $\frac{\frac{6020}{6125}}{4160}$ & $\frac{\frac{1333}{1102}}{522}$ & $\frac{\frac{336}{296}}{205}$ & $\frac{179}{\frac{122}{87}}$ \\
\hline
\end{tabular}

WITH TRANSMOUNTAIN DIVERSIONS

\begin{tabular}{|c|c|c|c|c|c|c|c|c|c|c|c|c|c|}
\hline & $\begin{array}{l}A \\
B \\
C\end{array}$ & $\begin{array}{l}567 \\
623 \\
514\end{array}$ & $\begin{array}{l}575 \\
613 \\
567\end{array}$ & $\begin{array}{r}475 \\
617 \\
57 \\
\end{array}$ & $\begin{array}{r}388 \\
570 \\
41 \\
\end{array}$ & $\begin{array}{r}385 \\
546 \\
46 \\
\end{array}$ & $\frac{\frac{333}{344}}{96}$ & $\frac{\frac{367}{344}}{344}$ & $\frac{\frac{1858}{1720}}{1720}$ & $\frac{\frac{2303}{2146}}{1720}$ & $\begin{array}{l}2362 \\
2406 \\
2233\end{array}$ & $\begin{array}{r}1497 \\
1785 \\
918\end{array}$ & $\begin{array}{r}886 \\
1116 \\
595\end{array}$ \\
\hline & $\begin{array}{l}A \\
B \\
C\end{array}$ & $\begin{array}{l}567 \\
592 \\
521\end{array}$ & $\begin{array}{l}563 \\
582 \\
538\end{array}$ & $\begin{array}{l}521 \\
567 \\
412\end{array}$ & $\begin{array}{l}498 \\
589 \\
274 \\
\end{array}$ & $\begin{array}{l}459 \\
560 \\
125 \\
\end{array}$ & $\frac{\frac{376}{359}}{204}$ & $\frac{\frac{380}{344}}{344}$ & $\frac{\frac{1960}{1720}}{1720}$ & $\frac{\frac{2518}{2150}}{1720}$ & $\begin{array}{l}2384 \\
2408 \\
2201\end{array}$ & $\begin{array}{l}1672 \\
1791 \\
1756\end{array}$ & $\begin{array}{r}937 \\
1128 \\
561\end{array}$ \\
\hline J & $\begin{array}{l}A \\
B \\
C\end{array}$ & $\begin{array}{l}563 \\
599 \\
525\end{array}$ & $\begin{array}{l}561 \\
580 \\
539\end{array}$ & $\begin{array}{l}510 \\
563 \\
479\end{array}$ & $\begin{array}{r}448 \\
552 \\
68 \\
\end{array}$ & $\begin{array}{r}403 \\
527 \\
51\end{array}$ & $\frac{\frac{322}{344}}{85}$ & $\frac{\frac{363}{344}}{344}$ & $\frac{\frac{1928}{1720}}{1720}$ & $\frac{\frac{2341}{2059}}{1720}$ & $\begin{array}{l}2352 \\
2400 \\
2090\end{array}$ & $\begin{array}{l}1660 \\
1792 \\
1756\end{array}$ & $\begin{array}{r}933 \\
1121 \\
571\end{array}$ \\
\hline & $\begin{array}{l}A \\
B \\
C\end{array}$ & $\frac{\frac{293}{241}}{170}$ & $\frac{147}{\frac{96}{54}}$ & $\frac{122}{\frac{61}{44}}$ & $\frac{\frac{165}{162}}{52}$ & $\frac{\frac{182}{169}}{53}$ & $\frac{\frac{514}{427}}{136}$ & $\frac{\frac{2498}{2314}}{1462}$ & $\frac{\frac{6359}{6055}}{4226}$ & $\frac{\frac{5727}{5832}}{3866}$ & $\frac{1178}{\frac{872}{522}}$ & $\frac{\frac{334}{291}}{205}$ & $\frac{16}{12}$ \\
\hline
\end{tabular}


Table 41.--Summary of simulated historical monthly streamflows, control point 19 (Yampa River near Lily, Colo.),

with 100 percent of agricultural and no transmountain diversions, and with 100 percent of both agricultural and transmountain diversions, and including 100 percent of industrial and municipal diversions for all simulations

[FLOW VALUES: $A=M E A N ; B=M E D I A N ;$ and $C=80-P E R C E N T$ EXCEEDENCE. Underscored values are less than corresponding table 37 simulated historical conditions]

OP- FLOW

MONTHLY FLOWS, IN CUBIC FEET PER SECOND

TION VALUES

OCT. NOV. DEC. JAN. FEB. MAR. APR. MAY JUNE JULY AUG. SEPT

WITHOUT TRANSMOUNTAIN DIVERSIONS

$\begin{array}{llllllllllllll}\text { A } & \text { A } & 496 & 476 & 356 & 405 & 446 & \frac{305}{284} & \frac{349}{344} & \frac{1830}{1720} & \frac{2765}{2730} & 2951 & 1343 & 775 \\ \text { B } & 559 & 588 & \frac{354}{354} & 601 & 585 & 1456 & 574\end{array}$

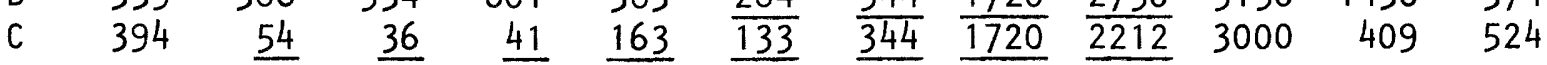

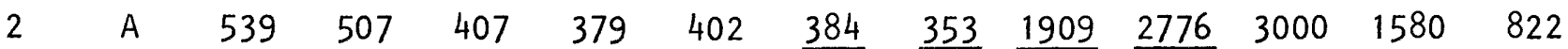

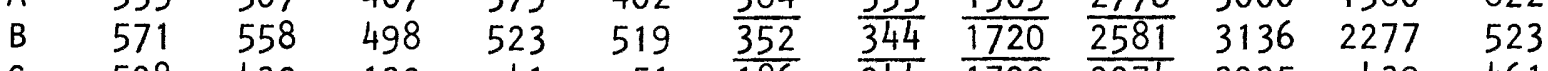

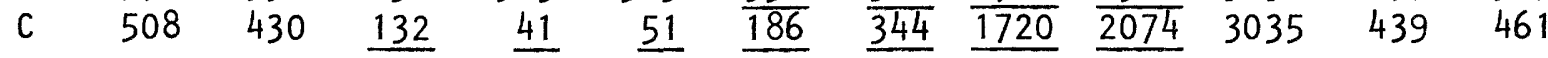

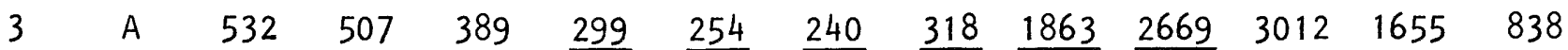

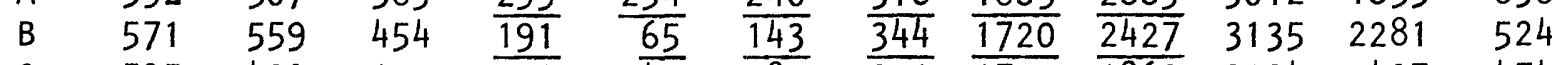

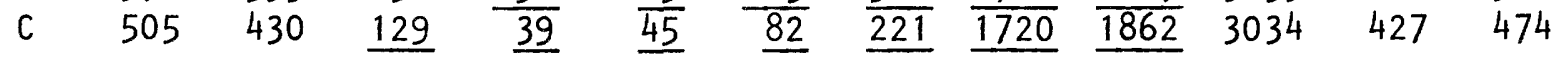

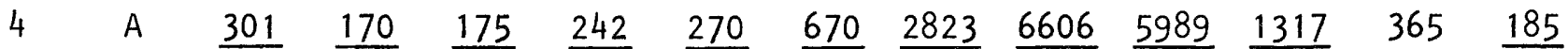

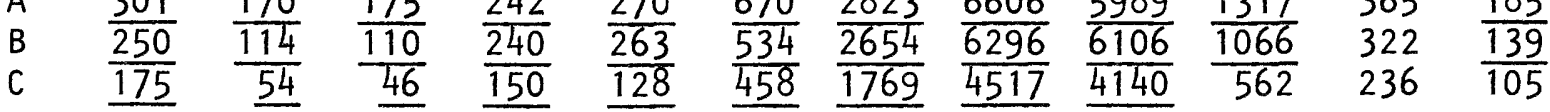

WITH TRANSMOUNTAIN DIVERSIONS

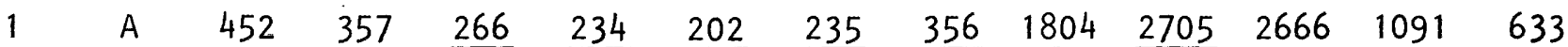

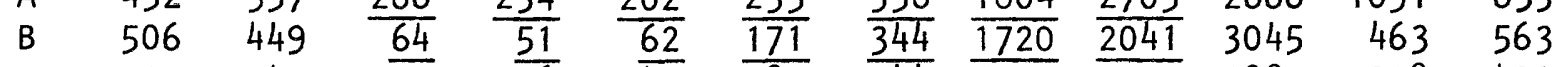

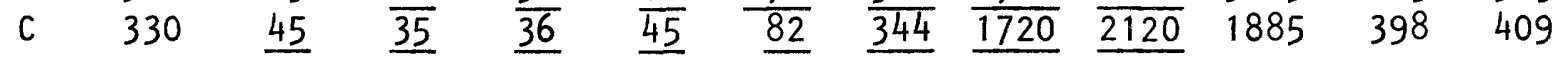

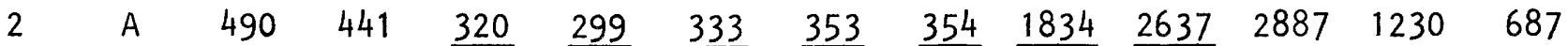

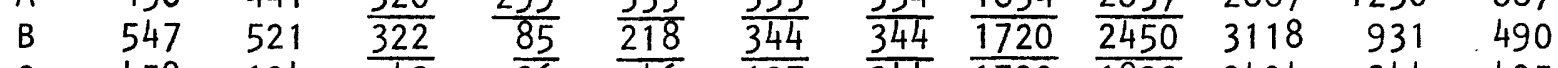

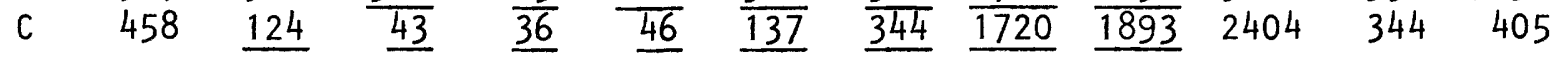

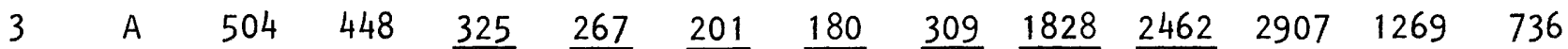

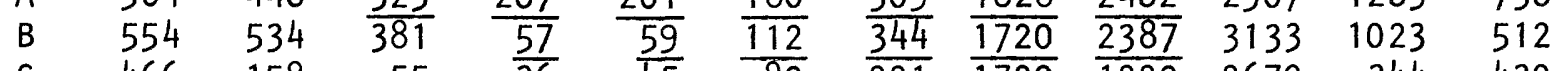

$\begin{array}{llllllllll}\text { C } 466 \quad \underline{158} \quad \underline{55} \quad \underline{36} \quad \underline{45} \quad \underline{80} \quad \underline{221} \quad \underline{1720} \quad \underline{1220} \quad 2670 \quad 344 & 430\end{array}$

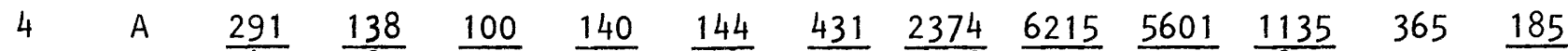

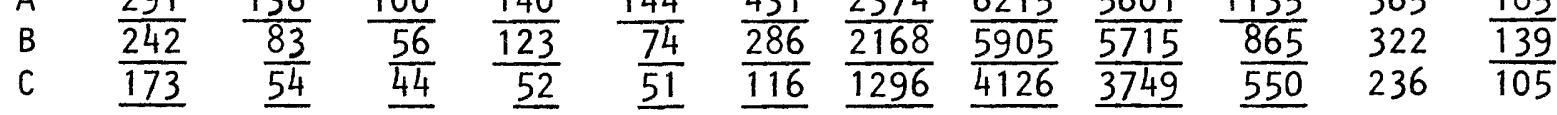


At the Yampa River near Lily site, the proposed Cross Mountain Reservoir is considered for reservoir-development options 1, 2, and 3 (table 2). Principally because of the possible large return flow from the Juniper Reservoir agricultural diversions, the monthly flow statistics at this site could reflect larger volumes than at the upstream site at Maybell (control point 18) (tables 33 through 36 and 38 through 41$)$. The flow statistics for this site also could indicate a more highly regulated annual-flow hydrograph than the historical discharges for reservoir-development options 1,2, and 3. Reservoir-development option 4 again followed more closely a historical annual-flow hydrograph because of the absence of the upstream Juniper and Cross Mountain Reservoirs. A hypothetical desired flow of $344 \mathrm{ft}^{3} / \mathrm{s}$, which is approximately equal to the historical summer average monthly flow, was established for this location. In many instances, the $344-\mathrm{ft}^{3} / \mathrm{s}$ desired flow was not met, particularly for the 100-percent water-use allocation level (table 41). For example, in reservoir-development option 4 with the 25-, 50-, 75-, and 100-percent water-use allocations, both with and without the transmountain diversions, the desired flow was not met during at least 6 months of each year (tables 38 through 41).

Model-simulated historical monthly streamflows for control point 43 (Little Snake River near Baggs, Wyo.) are presented in tables 42 through 46. In comparison to the streamflow-gaging-station measured data, simulated monthly mean streamflows for historical conditions without proposed transmountain diversions range from -8 to +8 percent and have an average absolute variation of 6 percent. This indicates that the model can reasonably simulate historical conditions at this control point (fig. 1), which is located downstream from the proposed Hog Park transmountain diversion and the proposed Sandstone and Pot Hook Reservoirs.

For reservoir development in option 1 without the Hog Park transmountain diversion, no change in flow was noticed from the historical conditions (tables 42 through 46) because neither Pot Hook nor Sandstone Reservoirs were considered for this option. Since there were no diversions for option 1 , the increase in allocation percentage similarly had no effect on the flow statistics (tables 42 through 46). In reservoir-development options 2, 3, and 4, both upstream reservoirs were considered and the flow statistics were reduced principally during the spring runoff of March, April, May, and June. Reservoir-development option-4 flow statistics were the most regular with less flow during the spring runoff and more flow during the summer and winter. By regulating the monthly flows at this location, additional flows also might be supplied during the low-flow months to the downstream Dinosaur National Monument.

Simulated historical streamflow statistics at control point 43 most significantly reflected the potential effects of proposed withdrawals by the Hog Park transmountain diversion during April through July. The effects of this diversion can most easily be seen in table 42 when 100-percent water-use allocation of transmountain diversion was added, resulting in zero flow for the month of July during many years. 
Table 42.--Summary of monthly streamflows, control point 43 (Little Snake River near Baggs, Wyo.), for simulated historical conditions, including 100 percent of transmountain diversions, and for historical conditions

[FLOW VALUES: $A=M E A N ; B=M E D I A N$; and $C=80-P E R C E N T$ EXCEEDENCE. Underscored values are less than historical conditions without transmountain diversions]

FLOW MONTHLY FLOWS, IN CUBIC FEET PER SECOND

VALUES OCT. NOV. DEC. JAN. FEB. MAR. APR. MAY JUNE JULY AUG. SEPT SIMULATED HISTORICAL CONDITIONS

$\begin{array}{rrrrrrrrrrrrr}\text { A } & 64 & 93 & 83 & 77 & 96 & 285 & 1069 & 2296 & 1841 & 185 & 28 & 34 \\ \text { B } & 49 & 78 & 78 & 66 & 88 & 222 & 847 & 2268 & 1805 & 65 & 18 & 17 \\ \text { C } & 25 & 54 & 55 & 54 & 55 & 140 & 446 & 1369 & 889 & 21 & 8 & 10\end{array}$

SIMULATED HISTORICAL CONDITIONS WITH 100 PERCENT OF TRANSMOUNTAIN DIVERSIONS

$\begin{array}{lllllllllllll}\text { A } & 64 & 93 & 83 & 77 & 96 & 285 & \underline{949} & \frac{2168}{730} & \frac{1715}{2140} & \frac{87}{1672} & 28 & 34 \\ \text { B } & 49 & 78 & 78 & 66 & 88 & 222 & \underline{730} & 18 & 17 \\ \text { C } & 25 & 54 & 55 & 54 & 55 & 140 & \underline{328} & \underline{1241} & \underline{761} & \underline{0} & 8 & 10\end{array}$

HISTORICAL STREAMFLOWS CALCULATED FROM GAGING-STATION RECORDS

\begin{tabular}{rrrrrrrrrrrrr} 
A & 66 & 96 & 85 & 79 & 95 & 348 & 1017 & 2482 & 1890 & 196 & 26 & 37 \\
B & 50 & 86 & 78 & 74 & 88 & 260 & 920 & 2412 & 1806 & 80 & 14 & 6 \\
$\mathrm{C}$ & 17 & 54 & 55 & 55 & 56 & 159 & 576 & 1408 & 968 & 21 & 3 & 1 \\
\hline
\end{tabular}


Table 43.--Summary of simulated historical monthly streamflows, control point 43 (Little snake River near Baggs, Wyo.),

with 25 percent of agricultural and no transmountain diversions, and with 25 percent of both agricultural and transmountain diversions, and including 100 percent of industrial and municipal diversions for all simulations

[FLOW VALUES: $A=M E A N ; B=M E D I A N ;$ and $C=80-P E R C E N T$ EXCEEDENCE. Underscored values are less than corresponding table 42 historical conditions]

\begin{tabular}{|c|c|c|c|c|c|c|c|c|c|c|c|c|c|}
\hline \multirow{2}{*}{$\begin{array}{l}\text { OP- } \\
\text { TION }\end{array}$} & \multirow{2}{*}{$\begin{array}{l}\text { FLOW } \\
\text { VALUES }\end{array}$} & \multicolumn{12}{|c|}{ MONTHLY FLOWS, IN CUBIC FEET PER SECOND } \\
\hline & & OCT. & NOV. & DEC. & JAN. & FEB. & MAR. & APR. & MAY & JUNE & JULY & AUG. & SEPT \\
\hline
\end{tabular}

WITHOUT TRANSMOUNTAIN DIVERSIONS

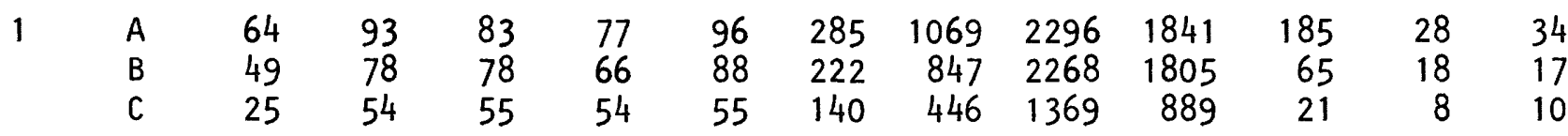

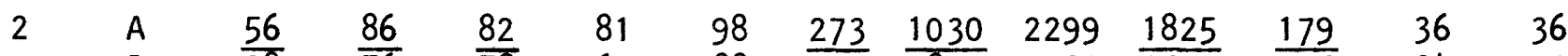

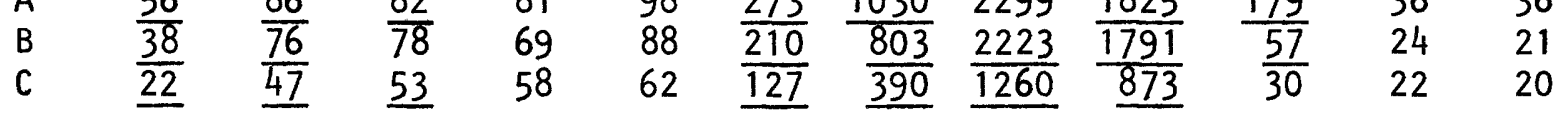

$\begin{array}{lllllllllllll}\text { A } & \frac{54}{35} & \frac{84}{71} & \frac{79}{76} & 77 & 96 & \frac{275}{21} & \frac{1038}{814} & 2302 & \frac{1825}{17} & \frac{179}{57} & 35 & 36 \\ \text { B } & \frac{35}{20} & \frac{75}{45} & \frac{75}{52} & 54 & \frac{85}{60} & \frac{213}{131} & \frac{814}{403} & \frac{2250}{1307} & \frac{1791}{873} & \frac{57}{30} & 22 & 19\end{array}$

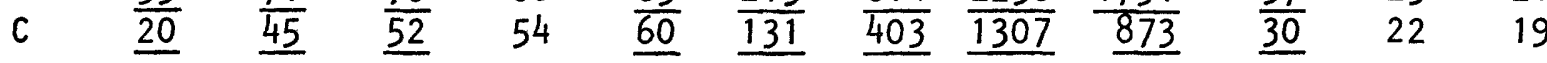

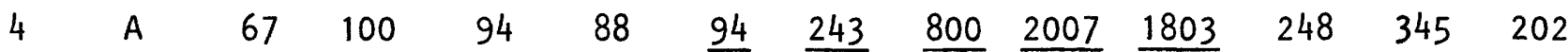

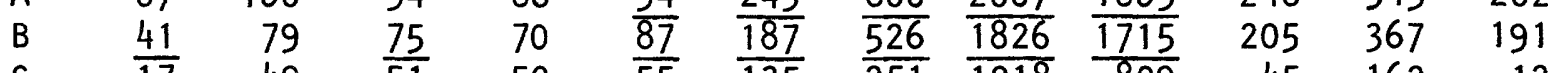

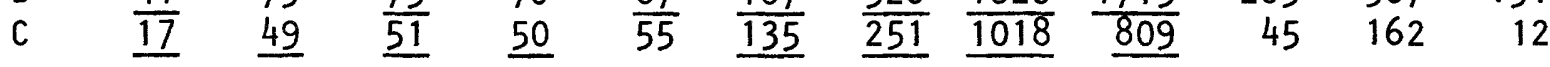

WITH TRANSMOUNTAIN DIVERSIONS

$\begin{array}{rlllllllllllll}1 & \text { A } & 64 & 93 & 83 & 77 & 96 & 285 & 1037 & \frac{2264}{2236} & \frac{1809}{1773} & \frac{153}{33} & 18 & 17 \\ \text { B } & 49 & 78 & 78 & 66 & 88 & 222 & \underline{815} & \frac{2234}{13} & \frac{857}{0} & \underline{0} & 8 & 10\end{array}$

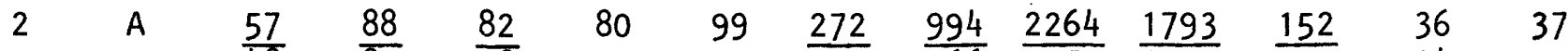

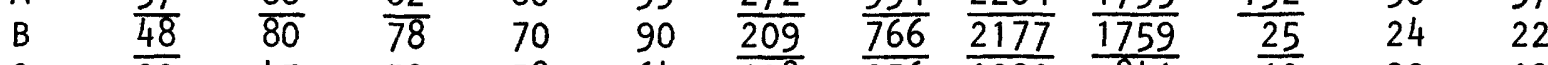

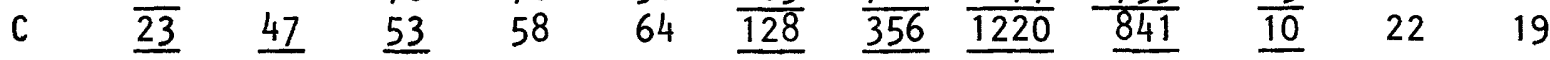

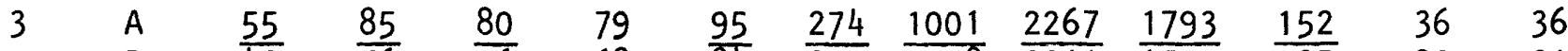

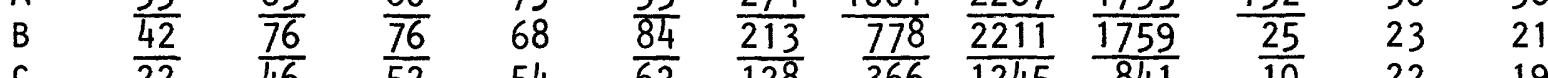

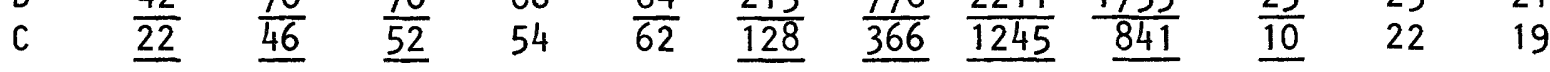

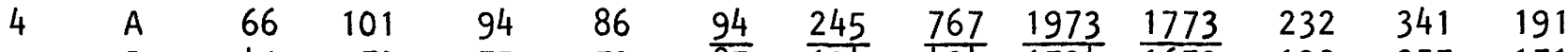

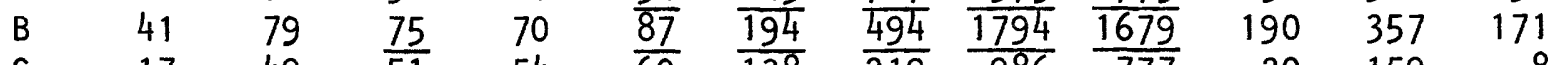

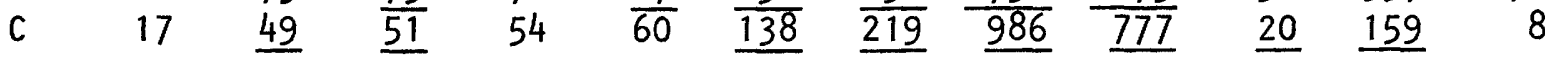


Table 44.--Summary of simulated historical monthly streamflows, control point 43 (Little Snake River near Baggs, Wyo.),

with 50 percent of agricultural and no transmountain diversions, and with 50 percent of both agricultural and transmountain diversions, and including 100 percent of industrial and municipal diversions for all simulations

[FLOW VALUES: $A=M E A N ; B=M E D I A N$; and $C=80-P E R C E N T$ EXCEEDENCE. Underscored values are less than corresponding table 42 historical conditions]

\begin{tabular}{|c|c|c|c|c|c|c|c|c|c|c|c|c|c|}
\hline \multirow{2}{*}{$\begin{array}{l}\text { OP- } \\
\text { TION }\end{array}$} & \multirow{2}{*}{$\begin{array}{c}\text { FLOW } \\
\text { VALUES }\end{array}$} & \multicolumn{12}{|c|}{ MONTHLY FLOWS, IN CUBIC FEET PER SECOND } \\
\hline & & OCT. & NOV. & DEC. & JAN. & FEB. & MAR. & APR. & MAY & JUNE & JULY & AUG. & SEPT \\
\hline & \multicolumn{13}{|c|}{ WITHOUT TRANSMOUNTAIN DIVERSIONS } \\
\hline 1 & $\begin{array}{l}A \\
B \\
C\end{array}$ & $\begin{array}{l}64 \\
49 \\
25\end{array}$ & $\begin{array}{l}93 \\
78 \\
54\end{array}$ & $\begin{array}{l}83 \\
78 \\
55\end{array}$ & $\begin{array}{l}77 \\
66 \\
54\end{array}$ & $\begin{array}{l}96 \\
88 \\
55\end{array}$ & $\begin{array}{l}285 \\
222 \\
140\end{array}$ & $\begin{array}{r}1069 \\
847 \\
446\end{array}$ & $\begin{array}{l}2296 \\
2268 \\
1369\end{array}$ & $\begin{array}{r}1841 \\
1805 \\
889\end{array}$ & $\begin{array}{r}185 \\
65 \\
21\end{array}$ & $\begin{array}{r}28 \\
18 \\
8\end{array}$ & $\begin{array}{l}34 \\
17 \\
10\end{array}$ \\
\hline 2 & $\begin{array}{l}A \\
B \\
C\end{array}$ & $\begin{array}{l}\frac{56}{40} \\
26\end{array}$ & $\begin{array}{l}93 \\
89 \\
56\end{array}$ & $\begin{array}{l}94 \\
85 \\
70\end{array}$ & $\begin{array}{l}90 \\
92 \\
52 \\
\end{array}$ & $\begin{array}{r}102 \\
89 \\
54 \\
\end{array}$ & $\frac{\frac{265}{205}}{135}$ & $\frac{\frac{967}{751}}{\underline{323}}$ & $\frac{\frac{2256}{2125}}{1206}$ & $\frac{\frac{1813}{1779}}{860}$ & $\begin{array}{r}194 \\
78 \\
59\end{array}$ & $\begin{array}{l}54 \\
45 \\
45\end{array}$ & $\begin{array}{l}41 \\
26 \\
26\end{array}$ \\
\hline 3 & $\begin{array}{l}A \\
B \\
C\end{array}$ & $\frac{\frac{54}{38}}{21}$ & $\begin{array}{l}\frac{90}{85} \\
49 \\
\end{array}$ & $\begin{array}{l}87 \\
\frac{77}{58}\end{array}$ & $\begin{array}{l}83 \\
75 \\
58\end{array}$ & $\begin{array}{l}97 \\
88 \\
57\end{array}$ & $\frac{\frac{267}{204}}{136}$ & $\frac{\frac{980}{768}}{364}$ & $\begin{array}{l}\frac{2266}{2142} \\
1206 \\
\end{array}$ & $\frac{\frac{1813}{1779}}{860}$ & $\begin{array}{r}194 \\
78 \\
59\end{array}$ & $\begin{array}{l}54 \\
45 \\
45\end{array}$ & $\begin{array}{l}41 \\
26 \\
26\end{array}$ \\
\hline 4 & $\begin{array}{l}A \\
B \\
C\end{array}$ & $\frac{\frac{65}{40}}{15}$ & $\begin{array}{l}\frac{99}{80} \\
49 \\
\end{array}$ & $\frac{\frac{91}{75}}{51}$ & $\frac{86}{\frac{70}{50}}$ & $\frac{\frac{94}{87}}{55}$ & $\frac{\frac{242}{187}}{135}$ & $\frac{\frac{803}{528}}{255}$ & $\frac{\frac{2004}{1831}}{1022}$ & $\frac{\frac{1788}{1695}}{821}$ & $\begin{array}{r}249 \\
217 \\
65\end{array}$ & $\begin{array}{l}340 \\
355 \\
146\end{array}$ & $\begin{array}{r}177 \\
141 \\
12\end{array}$ \\
\hline
\end{tabular}

WITH TRANSMOUNTAIN DIVERSIONS

$\begin{array}{rrrrrrrrrrrrrrr}1 & \text { A } & 64 & 93 & 83 & 77 & 96 & 285 & \frac{1006}{2232} & \frac{1777}{2204} & \frac{125}{1741} & 28 & 34 \\ \text { B } & 49 & 78 & 78 & 66 & 88 & 222 & \underline{783} & \frac{22}{18} & 17 \\ \text { C } & 25 & 54 & 55 & 54 & 55 & 140 & \underline{382} & \underline{1305} & \underline{825} & \underline{0} & 8 & 10\end{array}$

$\begin{array}{llllllllllllll}2 & A & 56 & 98 & 100 & 92 & 101 & \frac{266}{816} & \frac{896}{286} & \frac{2179}{2033} & \frac{1750}{1715} & \frac{142}{32} & 54 & 41\end{array}$

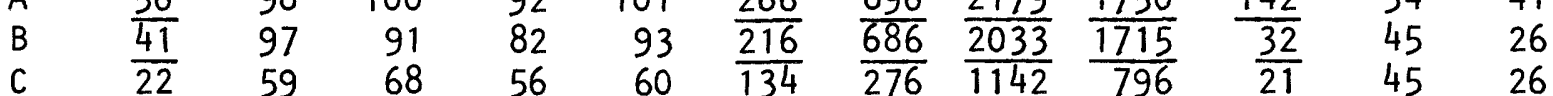

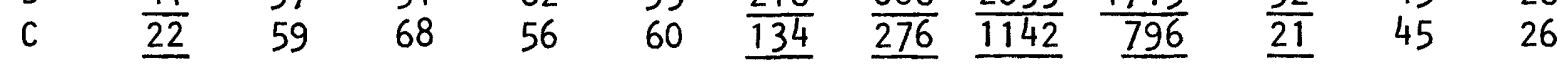

$\begin{array}{llllllllllllll}3 & \mathrm{~A} & 55 & 95 & 94 & 89 & 101 & \frac{265}{904} & 2186 & 1750 & \frac{142}{32} & 54 & 41\end{array}$

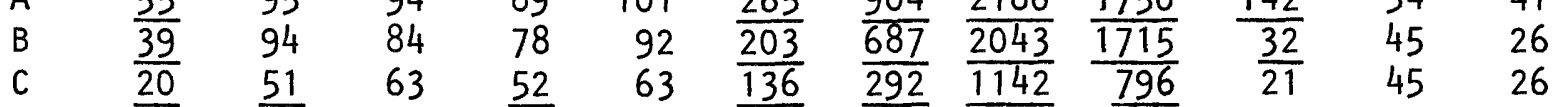

$\begin{array}{llllllllllllll}4 & A & 64 & 101 & 91 & 83 & \frac{94}{87} & \frac{247}{194} & \frac{738}{464} & \frac{1936}{1767} & \frac{1726}{1629} & 213 & 330 & 164\end{array}$

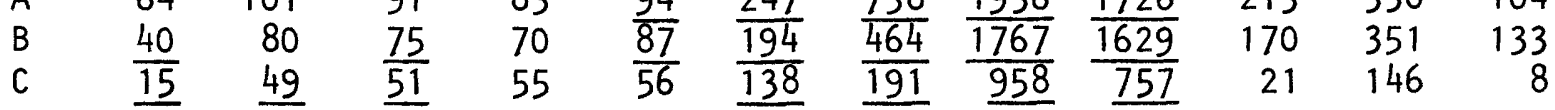


Table 45.--Summary of simulated historical monthly streamflows, control point 43 (Little Snake River near Baggs, Wyo.),

with 75 percent of agricultural and no transmountain diversions, and with 75 percent of both agricultural and transmountain diversions, and including 100 percent of industrial and municipal diversions for all simulations

[FLOW VALUES: $A=M E A N ; B=M E D I A N ;$ and $C=80-P E R C E N T$ EXCEEDENCE. Underscored values are less than corresponding table 42 historical conditions]

\begin{tabular}{|c|c|c|c|c|c|c|c|c|c|c|c|c|c|}
\hline \multirow{2}{*}{$\begin{array}{l}\text { OP- } \\
\text { TION }\end{array}$} & \multirow{2}{*}{$\begin{array}{l}\text { FLOW } \\
\text { VALUES }\end{array}$} & \multicolumn{12}{|c|}{ MONTHLY FLOWS, IN CUBIC FEET PER SECOND } \\
\hline & & OCT. & NOV. & DEC. & JAN. & FEB . & MAR. & APR. & MAY & JUNE & JULY & AUG. & SEPT \\
\hline \multicolumn{14}{|c|}{ WITHOUT TRANSMOUNTAIN DIVERSIONS } \\
\hline 1 & $\begin{array}{l}A \\
B \\
C\end{array}$ & $\begin{array}{l}64 \\
49 \\
25\end{array}$ & $\begin{array}{l}93 \\
78 \\
54\end{array}$ & $\begin{array}{l}83 \\
78 \\
55\end{array}$ & $\begin{array}{l}77 \\
66 \\
54\end{array}$ & $\begin{array}{l}96 \\
88 \\
55\end{array}$ & $\begin{array}{l}285 \\
222 \\
140\end{array}$ & $\begin{array}{r}1069 \\
847 \\
446\end{array}$ & $\begin{array}{l}2296 \\
2268 \\
1369\end{array}$ & $\begin{array}{r}1841 \\
1805 \\
889\end{array}$ & $\begin{array}{r}185 \\
65 \\
21\end{array}$ & $\begin{array}{r}28 \\
18 \\
8\end{array}$ & $\begin{array}{l}34 \\
17 \\
10\end{array}$ \\
\hline 2 & $\begin{array}{l}A \\
B \\
C\end{array}$ & $\frac{\frac{55}{39}}{20}$ & $\begin{array}{r}120 \\
125 \\
62\end{array}$ & $\begin{array}{r}141 \\
134 \\
84\end{array}$ & $\begin{array}{r}134 \\
134 \\
73\end{array}$ & $\begin{array}{r}118 \\
101 \\
62\end{array}$ & $\frac{\frac{259}{217}}{130}$ & $\frac{\frac{874}{647}}{317}$ & $\frac{\frac{2131}{1789}}{1158}$ & $\frac{\frac{1793}{1737}}{848}$ & $\begin{array}{r}216 \\
106 \\
89\end{array}$ & $\begin{array}{l}76 \\
67 \\
67\end{array}$ & $\begin{array}{l}54 \\
40 \\
40\end{array}$ \\
\hline 3 & $\begin{array}{l}A \\
B \\
C\end{array}$ & $\frac{\frac{54}{40}}{20}$ & $\begin{array}{r}118 \\
125 \\
58\end{array}$ & $\begin{array}{l}153 \\
161 \\
108\end{array}$ & $\begin{array}{r}137 \\
143 \\
85\end{array}$ & $\begin{array}{r}123 \\
106 \\
67\end{array}$ & $\begin{array}{l}\frac{266}{222} \\
131 \\
\end{array}$ & $\frac{\frac{876}{634}}{381}$ & $\frac{\frac{2109}{1752}}{1158}$ & $\frac{\frac{1789}{1737}}{848}$ & $\begin{array}{r}216 \\
106 \\
89\end{array}$ & $\begin{array}{l}76 \\
67 \\
67\end{array}$ & $\begin{array}{l}54 \\
40 \\
40\end{array}$ \\
\hline 4 & $\begin{array}{l}A \\
B \\
C\end{array}$ & $\frac{\frac{61}{38}}{16}$ & $\begin{array}{l}99 \\
78 \\
49 \\
\end{array}$ & $\begin{array}{l}89 \\
75 \\
51 \\
\end{array}$ & $\begin{array}{l}84 \\
70 \\
50 \\
\end{array}$ & $\frac{\frac{94}{87}}{55}$ & $\frac{\frac{242}{187}}{138}$ & $\frac{\frac{805}{529}}{258}$ & $\frac{\frac{2001}{1835}}{1026}$ & $\frac{\frac{1778}{1673}}{824}$ & $\begin{array}{r}255 \\
228 \\
89\end{array}$ & $\begin{array}{l}322 \\
358 \\
140\end{array}$ & $\begin{array}{r}157 \\
102 \\
9\end{array}$ \\
\hline
\end{tabular}

WITH TRANSMOUNTAIN DIVERSIONS

\begin{tabular}{|c|c|c|c|c|c|c|c|c|c|c|c|c|c|}
\hline & $\begin{array}{l}A \\
B \\
C\end{array}$ & $\begin{array}{l}64 \\
49 \\
25\end{array}$ & $\begin{array}{l}93 \\
78 \\
54\end{array}$ & $\begin{array}{l}83 \\
78 \\
55\end{array}$ & $\begin{array}{l}77 \\
66 \\
54\end{array}$ & $\begin{array}{l}96 \\
88 \\
55\end{array}$ & $\begin{array}{l}285 \\
222 \\
140\end{array}$ & $\frac{\frac{975}{751}}{350}$ & $\frac{\frac{2200}{2172}}{1273}$ & $\frac{1746}{1709}$ & $\frac{104}{\frac{0}{0}}$ & $\begin{array}{r}28 \\
18 \\
8\end{array}$ & $\begin{array}{l}34 \\
17 \\
10\end{array}$ \\
\hline & $\begin{array}{l}A \\
B \\
C\end{array}$ & $\begin{array}{l}74 \\
57 \\
22 \\
\end{array}$ & $\begin{array}{r}126 \\
124 \\
61\end{array}$ & $\begin{array}{l}149 \\
140 \\
111\end{array}$ & $\begin{array}{r}124 \\
138 \\
62\end{array}$ & $\begin{array}{r}125 \\
115 \\
73\end{array}$ & $\begin{array}{l}\frac{267}{223} \\
143\end{array}$ & $\frac{\frac{776}{523}}{\frac{255}{}}$ & $\frac{\frac{1986}{1690}}{1040}$ & $\frac{\frac{1694}{1631}}{752}$ & $\frac{141}{\frac{46}{31}}$ & $\begin{array}{l}80 \\
67 \\
67\end{array}$ & $\begin{array}{l}65 \\
40 \\
40\end{array}$ \\
\hline & $\begin{array}{l}A \\
B \\
C\end{array}$ & $\begin{array}{l}70 \\
56 \\
22 \\
\end{array}$ & $\begin{array}{r}127 \\
128 \\
61\end{array}$ & $\begin{array}{l}153 \\
154 \\
114\end{array}$ & $\begin{array}{r}136 \\
148 \\
63\end{array}$ & $\begin{array}{r}130 \\
118 \\
67\end{array}$ & $\begin{array}{l}\frac{271}{223} \\
143\end{array}$ & $\frac{\frac{780}{522}}{313}$ & $\frac{\frac{1968}{1656}}{1031}$ & $\frac{\frac{1688}{1607}}{752}$ & $\frac{141}{\frac{46}{31}}$ & $\begin{array}{l}86 \\
67 \\
67\end{array}$ & $\begin{array}{l}40 \\
40\end{array}$ \\
\hline & $\begin{array}{l}A \\
B \\
C\end{array}$ & $\frac{\frac{59}{38}}{16}$ & $\begin{array}{r}103 \\
78 \\
49 \\
\end{array}$ & $\begin{array}{l}88 \\
75 \\
51 \\
\end{array}$ & $\begin{array}{l}81 \\
70 \\
55\end{array}$ & $\frac{\frac{94}{87}}{56}$ & $\frac{\frac{251}{200}}{138}$ & $\frac{\frac{709}{433}}{174}$ & $\frac{\frac{1899}{1739}}{930}$ & $\frac{\frac{1682}{1577}}{723}$ & $\begin{array}{r}196 \\
160 \\
31\end{array}$ & $\begin{array}{l}311 \\
354 \\
140\end{array}$ & $\begin{array}{r}87 \\
8\end{array}$ \\
\hline
\end{tabular}


Table 46.--Summary of simulated historical monthly streamflows, control point 43 (Little Snake River near Baggs, Wyo.),

with 100 percent of agricultural and no transmountain diversions, and with 100 percent of both agricultural and transmountain diversions, and including 100 percent of industrial and municipal diversions for all simulations

[FLOW VALUES: $A=M E A N ; B=M E D I A N ;$ and $C=80-P E R C E N T$ EXCEEDENCE. Underscored values are less than corresponding table 42 historical conditions]

\begin{tabular}{|c|c|c|c|c|c|c|c|c|c|c|c|c|c|}
\hline \multirow{2}{*}{$\begin{array}{l}\text { OP- } \\
\text { TION }\end{array}$} & \multirow{2}{*}{$\begin{array}{l}\text { FLOW } \\
\text { VALUES }\end{array}$} & \multicolumn{12}{|c|}{ MONTHLY FLOWS, IN CUBIC FEET PER SECOND } \\
\hline & & OCT. & NOV. & DEC. & JAN. & FEB. & MAR. & APR. & MAY & JUNE & JULY & AUG. & SEPT \\
\hline & \multicolumn{13}{|c|}{ WITHOUT TRANSMOUNTAIN DIVERSIONS } \\
\hline 1 & $\begin{array}{l}A \\
B \\
C\end{array}$ & $\begin{array}{l}64 \\
49 \\
25\end{array}$ & $\begin{array}{l}93 \\
78 \\
54\end{array}$ & $\begin{array}{l}83 \\
78 \\
55\end{array}$ & $\begin{array}{l}77 \\
66 \\
54\end{array}$ & $\begin{array}{l}96 \\
88 \\
55\end{array}$ & $\begin{array}{l}285 \\
222 \\
140\end{array}$ & $\begin{array}{r}1069 \\
847 \\
446\end{array}$ & $\begin{array}{l}2296 \\
2268 \\
1369\end{array}$ & $\begin{array}{r}1841 \\
1805 \\
889\end{array}$ & $\begin{array}{r}185 \\
75 \\
21\end{array}$ & $\begin{array}{r}28 \\
18 \\
8\end{array}$ & $\begin{array}{l}34 \\
17 \\
10\end{array}$ \\
\hline 2 & $\begin{array}{l}A \\
B \\
C\end{array}$ & $\begin{array}{l}88 \\
82 \\
23 \\
\end{array}$ & $\begin{array}{r}142 \\
139 \\
71\end{array}$ & $\begin{array}{r}143 \\
136 \\
82\end{array}$ & $\begin{array}{r}113 \\
104 \\
60\end{array}$ & $\begin{array}{r}109 \\
99 \\
54 \\
\end{array}$ & $\frac{\frac{256}{220}}{114}$ & $\frac{\frac{843}{563}}{342}$ & $\frac{\frac{2039}{1784}}{1078}$ & $\frac{\frac{1772}{1691}}{835}$ & $\begin{array}{l}239 \\
132 \\
119\end{array}$ & $\begin{array}{l}99 \\
89 \\
89\end{array}$ & $\begin{array}{l}76 \\
59 \\
53\end{array}$ \\
\hline 3 & $\begin{array}{l}A \\
B \\
C\end{array}$ & $\begin{array}{l}89 \\
82 \\
24\end{array}$ & $\begin{array}{r}140 \\
139 \\
66\end{array}$ & $\begin{array}{r}151 \\
150 \\
92\end{array}$ & $\begin{array}{r}120 \\
106 \\
62\end{array}$ & $\begin{array}{r}112 \\
100 \\
55\end{array}$ & $\frac{\frac{264}{215}}{140}$ & $\frac{\frac{847}{548}}{\underline{386}}$ & $\frac{\frac{2020}{1758}}{1078}$ & $\frac{\frac{1767}{1691}}{835}$ & $\begin{array}{l}238 \\
132 \\
119\end{array}$ & $\begin{array}{l}99 \\
89 \\
89\end{array}$ & $\begin{array}{l}74 \\
58 \\
53\end{array}$ \\
\hline 4 & $\begin{array}{l}A \\
B \\
C\end{array}$ & $\frac{\frac{58}{36}}{15}$ & $\begin{array}{l}99 \\
76 \\
49 \\
\end{array}$ & $\begin{array}{l}86 \\
75 \\
51 \\
\end{array}$ & $\begin{array}{l}82 \\
70 \\
50 \\
\end{array}$ & $\frac{\frac{94}{87}}{55}$ & $\frac{\frac{241}{187}}{138}$ & $\frac{\frac{808}{531}}{261}$ & $\frac{\frac{1999}{1839}}{1030}$ & $\frac{\frac{1769}{1652}}{830}$ & $\begin{array}{l}264 \\
191 \\
119\end{array}$ & $\begin{array}{l}296 \\
328 \\
148\end{array}$ & $\begin{array}{r}139 \\
83 \\
8 \\
\end{array}$ \\
\hline
\end{tabular}

WITH TRANSMOUNTAIN DIVERSIONS

\begin{tabular}{|c|c|c|c|c|c|c|c|c|c|c|c|c|}
\hline 1 & $\begin{array}{l}A \\
B \\
C\end{array}$ & $\begin{array}{l}64 \\
49 \\
25\end{array}$ & $\begin{array}{l}93 \\
78 \\
54\end{array}$ & $\begin{array}{l}83 \\
78 \\
55\end{array}$ & $\begin{array}{l}77 \\
66 \\
54\end{array}$ & $\begin{array}{l}96 \\
88 \\
55\end{array}$ & $\begin{array}{l}285 \\
222 \\
140\end{array}$ & $\frac{\frac{949}{730}}{328}$ & $\frac{\frac{2168}{2140}}{\frac{1241}{2}}$ & $\frac{\frac{1715}{1677}}{761}$ & $\frac{87}{\frac{0}{0}}$ & $\begin{array}{r}28 \\
18 \\
8\end{array}$ \\
\hline 2 & $\begin{array}{l}A \\
B \\
C\end{array}$ & $\begin{array}{r}102 \\
92 \\
39\end{array}$ & $\begin{array}{r}141 \\
142 \\
64\end{array}$ & $\begin{array}{r}137 \\
123 \\
70\end{array}$ & $\begin{array}{r}110 \\
90 \\
58\end{array}$ & $\begin{array}{r}112 \\
90 \\
62\end{array}$ & $\frac{\frac{250}{207}}{107}$ & $\frac{737}{449}$ & $\frac{\frac{1878}{1630}}{884}$ & $\frac{\frac{1641}{1554}}{707}$ & $\begin{array}{r}145 \\
66 \\
47\end{array}$ & $\begin{array}{r}106 \\
89 \\
89\end{array}$ \\
\hline 3 & $\begin{array}{l}A \\
B \\
C\end{array}$ & $\begin{array}{l}95 \\
87 \\
39\end{array}$ & $\begin{array}{r}148 \\
153 \\
66\end{array}$ & $\begin{array}{r}146 \\
136 \\
68\end{array}$ & $\begin{array}{r}114 \\
94 \\
56\end{array}$ & $\begin{array}{r}115 \\
97 \\
67\end{array}$ & $\frac{\frac{271}{220}}{140}$ & $\frac{741}{479}$ & $\frac{\frac{1855}{1630}}{874}$ & $\frac{1636}{1556}$ & $\begin{array}{r}144 \\
66 \\
47\end{array}$ & $\begin{array}{r}103 \\
89 \\
89\end{array}$ \\
\hline 4 & $\begin{array}{l}A \\
B \\
C\end{array}$ & $\frac{\frac{58}{36}}{15}$ & $\begin{array}{r}105 \\
76 \\
49 \\
\end{array}$ & $\begin{array}{l}85 \\
75 \\
51 \\
\end{array}$ & $\begin{array}{l}78 \\
68 \\
55\end{array}$ & $\begin{array}{l}\frac{95}{88} \\
56\end{array}$ & $\frac{\frac{254}{201}}{139}$ & $\frac{\frac{683}{403}}{177}$ & $\frac{\frac{1864}{1711}}{889}$ & $\frac{\frac{1641}{1524}}{\underline{702}}$ & $\begin{array}{r}184 \\
140 \\
48\end{array}$ & $\begin{array}{l}286 \\
323 \\
136\end{array}$ \\
\hline
\end{tabular}


Model-simulated historical monthly streamflow statistics for control point 41 (Yampa River near Deerlodge Park, Colo.) are presented in tables 47 through 51. This site is located downstream from the confluence of the Yampa and the Little Snake Rivers near the entrance to Dinosaur National Monument (fig. 1). Flow statistics given in tables 47 through 51 less than historical values are underscored. Although no streamflow record is available at this site, the historical simulated flow statistics should conform to the actual streamflows as there was close agreement for the nearest upstream streamflow-gaging stations, Yampa River at Maybell (control point 18) and Little Snake River near Lily (control point 42).

Table 47.--Summary of simulated historical monthly streamflows, control point 41 (Yampa River near Deerlodge Park, Colo.), for historical conditions and with 100 percent of transmountain diversions

[FLOW VALUES: $A=M E A N ; B=M E D I A N$; and $C=80-P E R C E N T$ EXCEEDENCE. Underscored values are less than historical conditions without transmountain diversions]

\begin{tabular}{|c|c|c|c|c|c|c|c|c|c|c|c|c|}
\hline \multirow{2}{*}{$\begin{array}{l}\text { FLOW } \\
\text { VALUES }\end{array}$} & \multicolumn{12}{|c|}{ MONTHLY FLOWS, IN CUBIC FEET PER SECOND } \\
\hline & OCT. & NOV. & DEC. & JAN. & FEB. & MAR. & APR & MAY & JUNE & JULY & AUG. & SEPT \\
\hline \multicolumn{13}{|c|}{ SIMULATED HISTORICAL CONDITIONS } \\
\hline $\begin{array}{l}A \\
B \\
C\end{array}$ & $\begin{array}{l}517 \\
458 \\
279\end{array}$ & $\begin{array}{l}513 \\
483 \\
343\end{array}$ & $\begin{array}{l}461 \\
445 \\
361\end{array}$ & $\begin{array}{l}424 \\
402 \\
346\end{array}$ & $\begin{array}{l}499 \\
435 \\
375\end{array}$ & $\begin{array}{r}1223 \\
933 \\
853\end{array}$ & $\begin{array}{l}4359 \\
4030 \\
2527\end{array}$ & $\begin{array}{l}9932 \\
9408 \\
6688\end{array}$ & 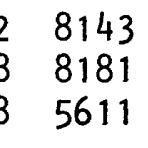 & $\begin{array}{r}1721 \\
1410 \\
646\end{array}$ & $\begin{array}{l}424 \\
340 \\
181\end{array}$ & $\begin{array}{l}295 \\
248 \\
114\end{array}$ \\
\hline \multicolumn{2}{|c|}{ SIMULATED $\mathrm{H}$} & STORIC & $A L \quad C O N$ & ITIONS & WITH & 100 & PERCENT & OF T & TRANSMOL & NTAIN & DIVERS & IONS \\
\hline $\begin{array}{l}A \\
B \\
C\end{array}$ & $\frac{\frac{438}{363}}{211}$ & $\frac{408}{\frac{400}{189}}$ & $\frac{\frac{374}{367}}{267}$ & $\frac{\frac{346}{324}}{\frac{264}{2}}$ & $\frac{\frac{421}{357}}{297}$ & $\frac{1145}{\frac{855}{775}}$ & $\frac{\frac{3856}{3511}}{2105}$ & $\begin{array}{l}\frac{9413}{8890} \\
6169 \\
\end{array}$ & $\frac{3}{5} \frac{7631}{7662}$ & $\frac{\frac{1368}{1029}}{382}$ & $\frac{\frac{361}{281}}{131}$ & $\frac{\frac{227}{160}}{72}$ \\
\hline
\end{tabular}


Table 48.--Summary of simulated historical monthly streamflows, control point 41 (Yampa River near Deerlodge Park, Colo.), with 25 percent of agricultural and no transmountain diversions, and with 25 percent of both agricultural and transmountain diversions, and including 100 percent of industrial and municipal diversions for all simulations

[FLOW VALUES: $A=$ MEAN; $B=$ MEDIAN; and $C=80-P E R C E N T$ EXCEEDENCE. Underscored values are less than corresponding table 47 simulated historical conditions]

\begin{tabular}{|c|c|c|c|c|c|c|c|c|c|c|c|c|c|}
\hline \multirow{2}{*}{$\begin{array}{l}\text { OP- } \\
\text { TION }\end{array}$} & \multirow{2}{*}{$\begin{array}{c}\text { FLOW } \\
\text { VALUES }\end{array}$} & \multicolumn{12}{|c|}{ MONTHLY FLOWS, IN CUBIC FEET PER SECOND } \\
\hline & & OCT. & NOV. & DEC. & JAN. & FEB. & MAR. & APR. & MAY & JUNE & JULY & AUG. & SEPT \\
\hline \multicolumn{14}{|c|}{ WITHOUT TRANSMOUNTAIN DIVERSIONS } \\
\hline 1 & $\begin{array}{l}A \\
B \\
C\end{array}$ & $\begin{array}{l}740 \\
750 \\
750\end{array}$ & $\begin{array}{l}738 \\
750 \\
750\end{array}$ & $\begin{array}{l}738 \\
750 \\
750\end{array}$ & $\begin{array}{l}750 \\
750 \\
750\end{array}$ & $\begin{array}{l}742 \\
750 \\
750\end{array}$ & $\frac{\frac{863}{750}}{750}$ & $\frac{\frac{2052}{1690}}{961}$ & $\frac{\frac{7056}{6130}}{3484}$ & $\frac{\frac{6837}{6470}}{3725}$ & $\begin{array}{l}2235 \\
2030 \\
1869\end{array}$ & $\begin{array}{l}850 \\
806 \\
750\end{array}$ & $\begin{array}{l}761 \\
750 \\
750\end{array}$ \\
\hline 2 & $\begin{array}{l}A \\
B \\
C\end{array}$ & $\begin{array}{l}740 \\
750 \\
750\end{array}$ & $\begin{array}{l}738 \\
750 \\
750\end{array}$ & $\begin{array}{l}738 \\
750 \\
750\end{array}$ & $\begin{array}{l}750 \\
750 \\
750\end{array}$ & $\begin{array}{l}751 \\
750 \\
750\end{array}$ & $\frac{\frac{860}{750}}{750}$ & $\frac{2021}{\frac{1656}{924}}$ & $\frac{\frac{7452}{6722}}{\underline{3747}}$ & $\frac{\frac{7312}{7039}}{4040}$ & $\begin{array}{l}2244 \\
2013 \\
1875\end{array}$ & $\begin{array}{l}857 \\
822 \\
750\end{array}$ & $\begin{array}{l}760 \\
750 \\
750\end{array}$ \\
\hline 3 & $\begin{array}{l}A \\
B \\
C\end{array}$ & $\begin{array}{l}740 \\
750 \\
750\end{array}$ & $\begin{array}{l}738 \\
750 \\
750\end{array}$ & $\begin{array}{l}738 \\
750 \\
750\end{array}$ & $\begin{array}{l}738 \\
750 \\
750\end{array}$ & $\begin{array}{l}739 \\
750 \\
750\end{array}$ & $\frac{\frac{854}{750}}{750}$ & $\frac{\frac{2131}{1749}}{946}$ & $\frac{\frac{7217}{6403}}{3631}$ & $\frac{\frac{7113}{6702}}{3878}$ & $\begin{array}{l}2238 \\
2013 \\
1875\end{array}$ & $\begin{array}{l}860 \\
827 \\
750\end{array}$ & $\begin{array}{l}760 \\
750 \\
750\end{array}$ \\
\hline 4 & $\begin{array}{l}A \\
B \\
C\end{array}$ & $\frac{\frac{419}{339}}{207}$ & $\frac{\frac{378}{330}}{202}$ & $\frac{\frac{373}{347}}{267}$ & $\frac{\frac{361}{333}}{273}$ & $\frac{\frac{429}{367}}{317}$ & $\frac{1029}{\frac{750}{705}}$ & $\frac{\frac{3649}{3320}}{2058}$ & $\frac{\frac{9143}{8726}}{6232}$ & $\frac{7955}{8023}$ & $\frac{1708}{\frac{1303}{750}}$ & $\begin{array}{l}691 \\
750 \\
750\end{array}$ & $\begin{array}{r}407 \\
393 \\
97 \\
\end{array}$ \\
\hline
\end{tabular}

WITH TRANSMOUNTAIN DIVERSIONS

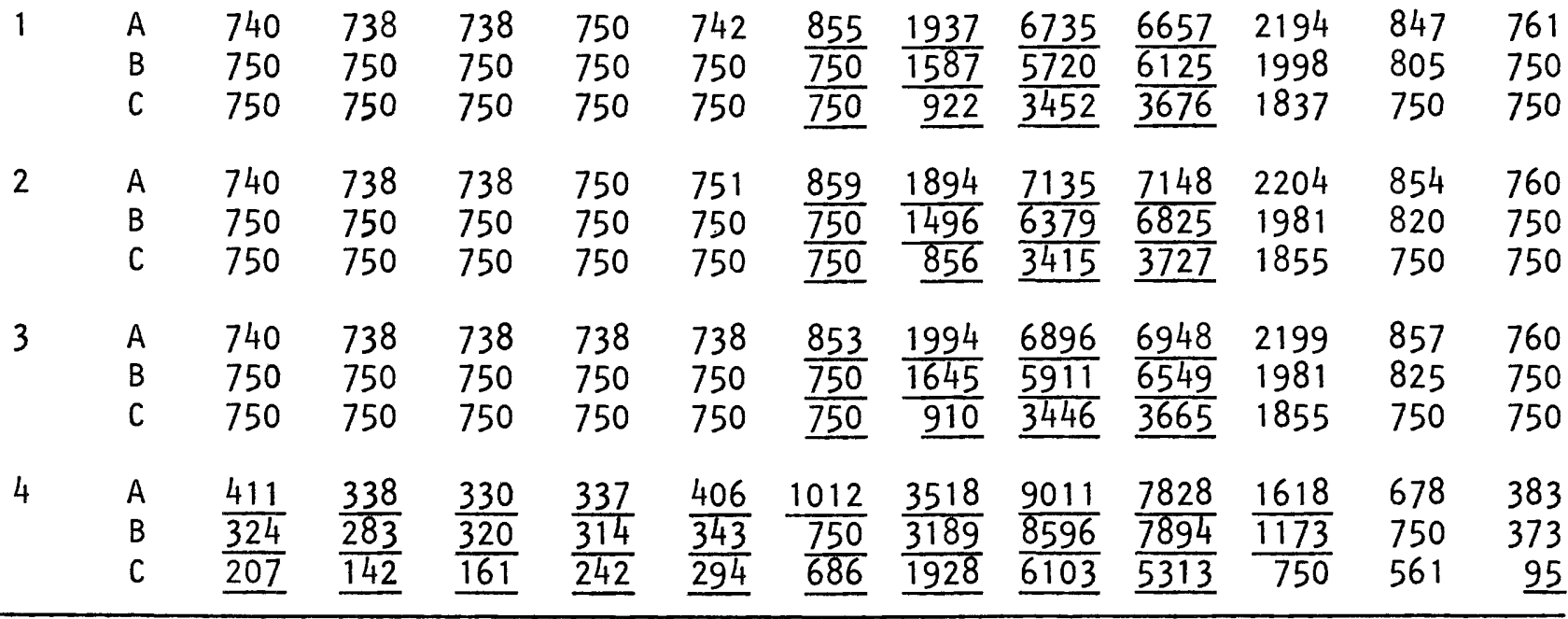


Table 49.--Summary of simulated historical monthly streamflows, control point 41 (Yampa River near Deerlodge Park, Colo.), with 50 percent of agricultural and no transmountain diversions, and with 50 percent of both agricultural and transmountain diversions, and including 100 percent of industrial and municipal diversions for all simulations

[FLOW VALUES: $A=M E A N ; B=M E D I A N ;$ and $C=80-P E R C E N T$ EXCEEDENCE. Underscored values are less than corresponding table 47 simulated historical conditions]

\begin{tabular}{|c|c|c|c|c|c|c|c|c|c|c|c|c|c|c|}
\hline \multirow{2}{*}{$\begin{array}{l}\text { OP- } \\
\text { TION }\end{array}$} & \multirow{2}{*}{$\begin{array}{l}\text { FLOW } \\
\text { VALUES }\end{array}$} & \multicolumn{4}{|c|}{ MONTHLY } & \multicolumn{2}{|c|}{ FLOWS, I } & \multicolumn{5}{|c|}{ CUBIC FEET PER SECOND } & \multirow[b]{2}{*}{$A \cup G$} & \multirow[b]{2}{*}{ SEPT } \\
\hline & & OCT. & NOV. & DEC. & JAN. & FEB. & MAR. & APR. & MAY & & UNE & JULY & & \\
\hline
\end{tabular}

WITHOUT TRANSMOUNTAIN DIVERSIONS

\begin{tabular}{|c|c|c|c|c|c|c|c|c|c|c|c|c|}
\hline & $\begin{array}{l}741 \\
750 \\
750\end{array}$ & $\begin{array}{l}738 \\
750 \\
750\end{array}$ & $\begin{array}{l}738 \\
750 \\
750\end{array}$ & $\begin{array}{l}750 \\
750 \\
750\end{array}$ & $\begin{array}{l}742 \\
750 \\
750\end{array}$ & $\begin{array}{l}\frac{860}{750} \\
750 \\
\end{array}$ & $\frac{\frac{1685}{1558}}{964}$ & $\frac{\frac{5763}{4708}}{3433}$ & $\frac{\frac{5790}{4870}}{3184}$ & $\begin{array}{l}2350 \\
2176 \\
1963\end{array}$ & $\begin{array}{l}1461 \\
1459 \\
1383\end{array}$ & $\begin{array}{l}972 \\
948 \\
905\end{array}$ \\
\hline & $\begin{array}{l}740 \\
750 \\
750\end{array}$ & $\begin{array}{l}738 \\
750 \\
750\end{array}$ & $\begin{array}{l}738 \\
750 \\
750\end{array}$ & $\begin{array}{l}750 \\
750 \\
750\end{array}$ & $\begin{array}{l}750 \\
750 \\
750\end{array}$ & $\frac{\frac{847}{750}}{750}$ & $\frac{\frac{1607}{1388}}{792}$ & $\frac{6006}{\frac{4749}{3344}}$ & $\frac{\frac{6416}{5485}}{3195}$ & $\begin{array}{l}2362 \\
2194 \\
1998\end{array}$ & $\begin{array}{l}1491 \\
1491 \\
1420\end{array}$ & $\begin{array}{l}980 \\
948 \\
919\end{array}$ \\
\hline & $\begin{array}{l}740 \\
750 \\
750\end{array}$ & $\begin{array}{l}738 \\
750 \\
750\end{array}$ & $\begin{array}{l}738 \\
750 \\
750\end{array}$ & $\begin{array}{l}738 \\
750 \\
750\end{array}$ & $\begin{array}{l}738 \\
750 \\
750\end{array}$ & $\frac{\frac{843}{750}}{750}$ & $\frac{1675}{\frac{1410}{835}}$ & $\frac{\frac{5808}{4875}}{3349}$ & $\frac{\frac{5091}{5157}}{\frac{3189}{}}$ & $\begin{array}{l}2360 \\
2194 \\
1998\end{array}$ & $\begin{array}{l}1494 \\
1491 \\
1421\end{array}$ & $\begin{array}{l}980 \\
947 \\
918\end{array}$ \\
\hline & $\frac{\frac{415}{327}}{211}$ & $\frac{\frac{350}{315}}{142}$ & $\frac{\frac{348}{343}}{190}$ & $\frac{\frac{362}{337}}{281}$ & $\frac{\frac{432}{371}}{319}$ & $\frac{1032}{\frac{750}{709}}$ & $\frac{\frac{3653}{3321}}{2067}$ & $\frac{\frac{9026}{8590}}{6191}$ & $\frac{7918}{\frac{7998}{5389}}$ & $\frac{\frac{1693}{1307}}{750}$ & $\begin{array}{l}694 \\
750 \\
638\end{array}$ & $\begin{array}{l}380 \\
341 \\
113\end{array}$ \\
\hline
\end{tabular}

WITH TRANSMOUNTAIN DIVERSIONS

$\begin{array}{llllllllllllll}1 & \text { A } & 741 & 738 & 738 & 738 & 752 & 852 & 1546 & 5292 & \underline{5204} & 2277 & 1423 & 971 \\ \text { B } & 750 & 750 & 750 & 750 & 750 & \underline{750} & \underline{1471} & \underline{4399} & \underline{4343} & 2125 & 1459 & 948 \\ \text { C } & 750 & 750 & 750 & 750 & 750 & \underline{750} & \underline{900} & \underline{3308} & \underline{3026} & 1907 & 1372 & 905\end{array}$

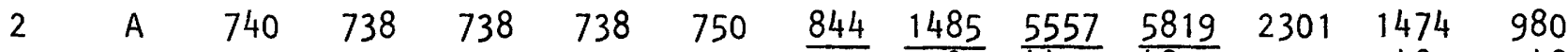

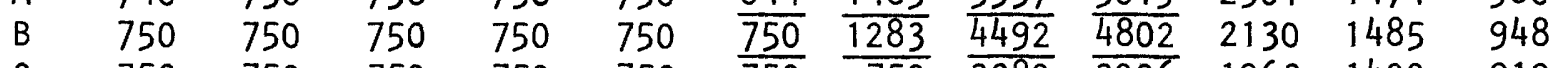

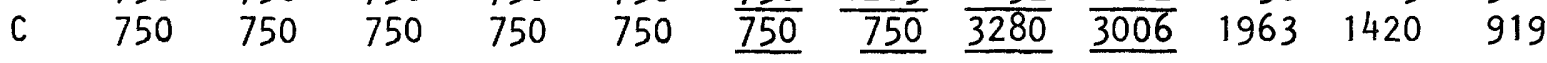

$\begin{array}{llllllllllllll}3 & \mathrm{~A} & 740 & 738 & 738 & 738 & 738 & 838 & 1530 & 5323 & 5484 & 2298 & 1474 & 980\end{array}$

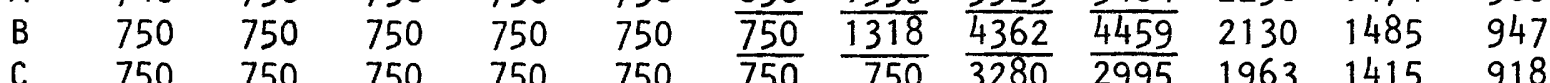

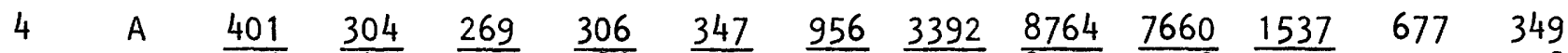

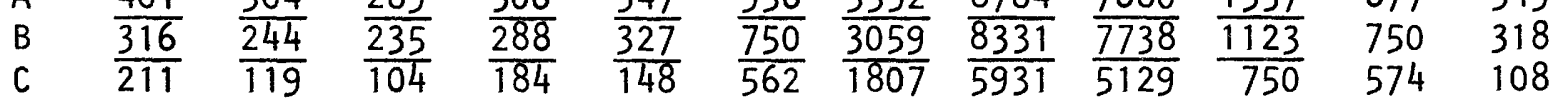


Table 50.--Summary of simulated historical monthly streamflows, control point 41 (Yampa River near Deerlodge Park, Colo.),

with 75 percent of agricultural and no transmountain diversions, and with 75 percent of both agricultural and transmountain diversions, and including 100 percent of industrial and municipal diversions for all simulations

[FLOW VALUES: $A=M E A N ; B=M E D I A N ;$ and $C=80-P E R C E N T$ EXCEEDENCE. Underscored values are less than corresponding table 47 simulated historical conditions]

OP- FLOW

MONTHLY FLOWS, IN CUBIC FEET PER SECOND

TION VALUES

OCT. NOV. DEC. JAN. FEB. MAR. APR. MAY JUNE JULY AUG. SEPT

WITHOUT TRANSMOUNTAIN DIVERSIONS

$\begin{array}{llllllllllllll}1 & \text { A } & 743 & 723 & 635 & 661 & 634 & 772 & 1502 & 4728 & 4680 & 2960 & 1986 & 1152 \\ & \text { B } & 750 & 750 & 750 & 750 & 750 & 750 & \frac{4356}{4411} & \frac{4326}{2908} & 2047 & 1264\end{array}$

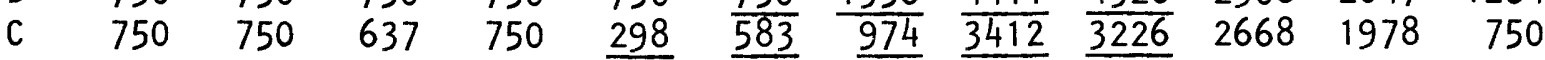

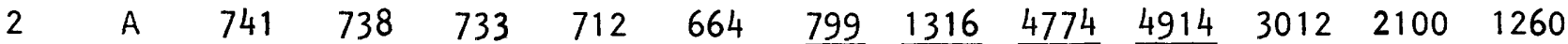

$\begin{array}{llllllllllll}\text { B } & 750 & 750 & 750 & 750 & 750 & \frac{750}{750} & \frac{1168}{4261} & \frac{4373}{33} & 2945 & 2099 & 1299\end{array}$

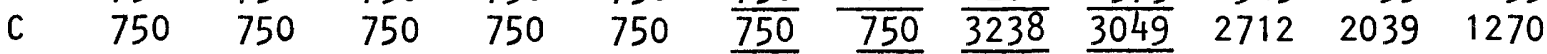

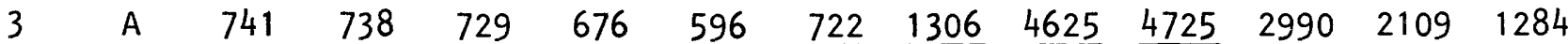

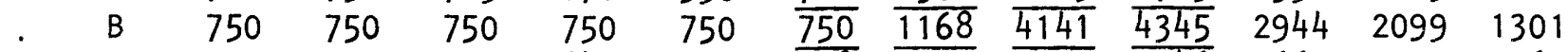

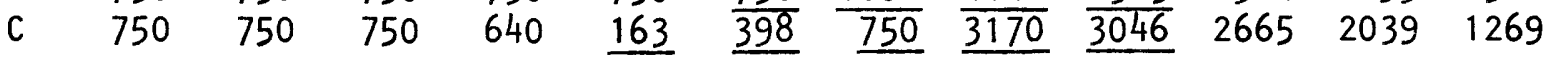

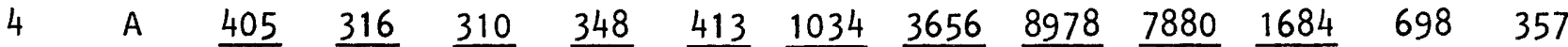

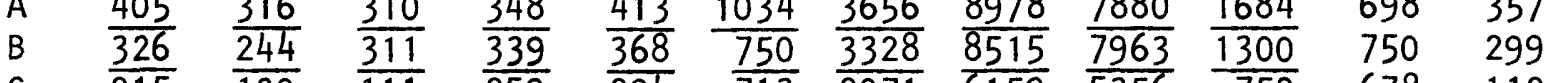

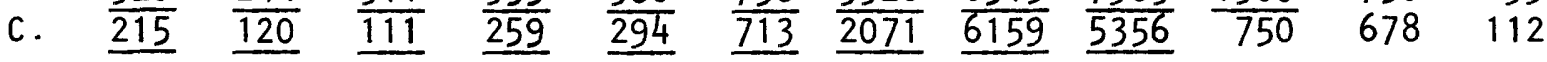

WITH TRANSMOUNTAIN DIVERSIONS

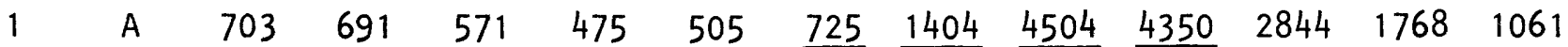

$\begin{array}{llllllllllll}\text { B } & 750 & 750 & 750 & 745 & 750 & \frac{750}{1260} & \underline{4315} & \frac{4166}{376} & 2807 & 2030 & 1251\end{array}$

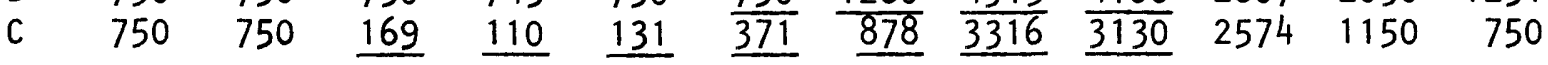

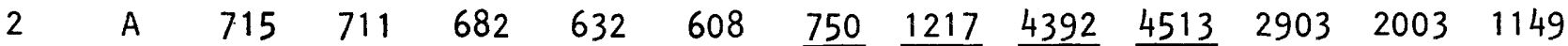

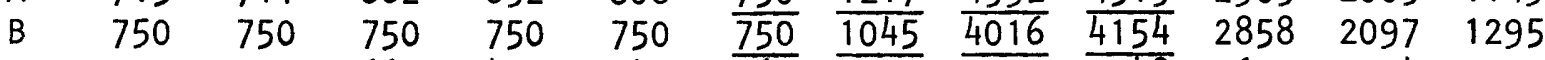

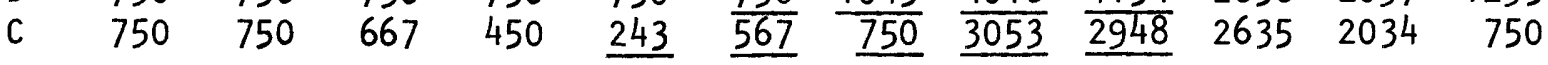

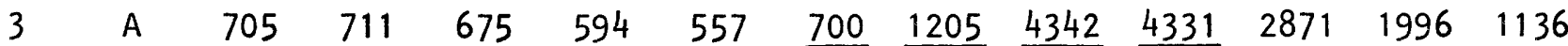

$\begin{array}{llllllllllll}\text { B } & 750 & 750 & 750 & 750 & 750 & \overline{750} & \overline{1044} & \frac{43469}{39658} & \frac{4058}{284} & 2097 & 1287\end{array}$

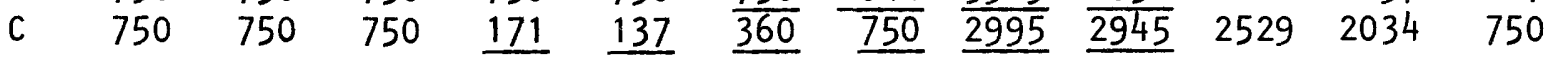

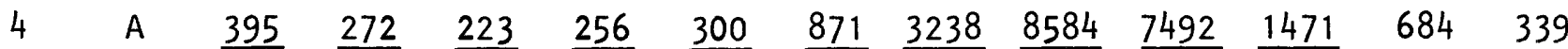

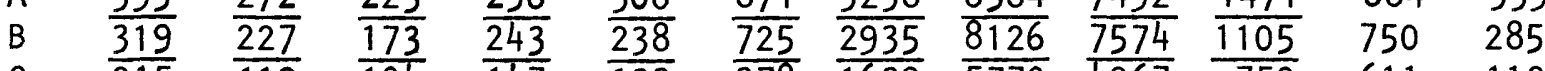

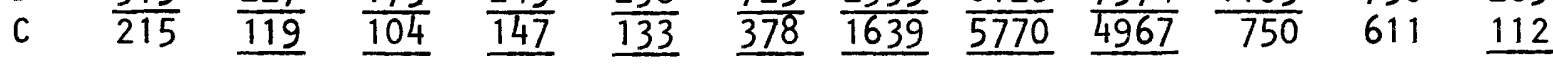


Table 51.--Summary of simulated historical monthly streamflows, control point 41 (Yampa River near Deerlodge Park, Colo.), with 100 percent of agricultural and no transmountain diversions, and with 100 percent of both agricultural and transmountain diversions, and including 100 percent of industrial and municipal diversions for all simulations

[FLOW VALUES: $A=M E A N ; B=M E D I A N ;$ and $C=80-P E R C E N T$ EXCEEDENCE. Underscored values are less than corresponding table 47 simulated historical conditions]

OP- FLOW

MONTHLY FLOWS, IN CUBIC FEET PER SECOND

TION VALUES

OCT. NOV. DEC. JAN. FEB. MAR. APR. MAY JUNE JULY AUG. SEPT

WITHOUT TRANSMOUNTAIN DIVERSIONS

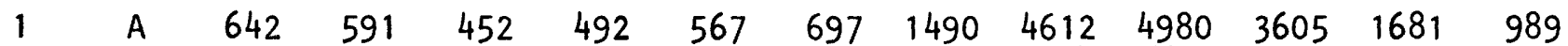

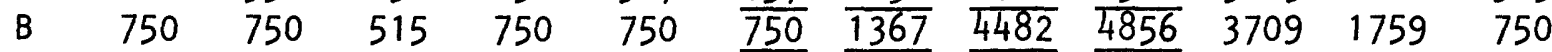

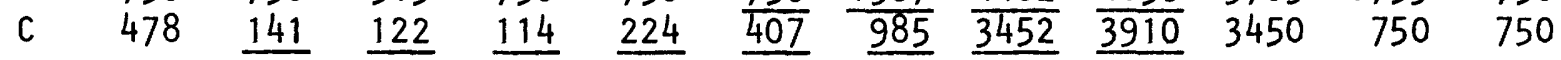

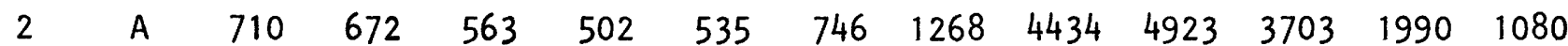

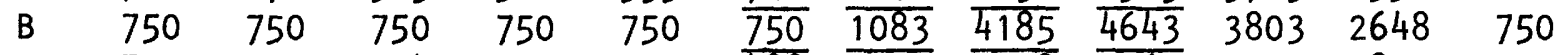

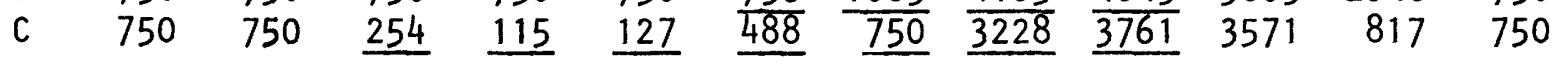

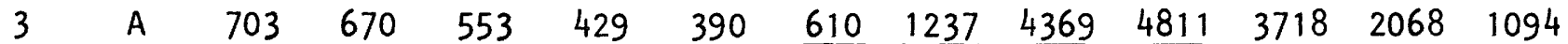

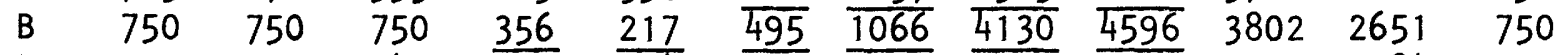

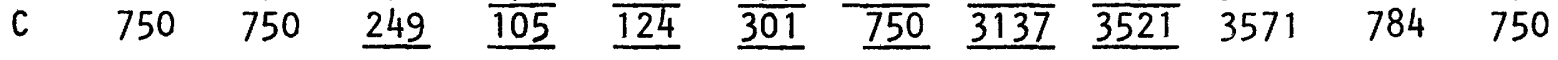

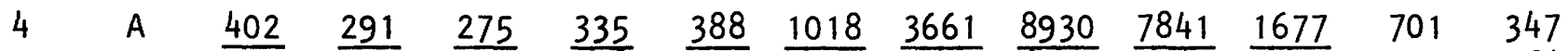

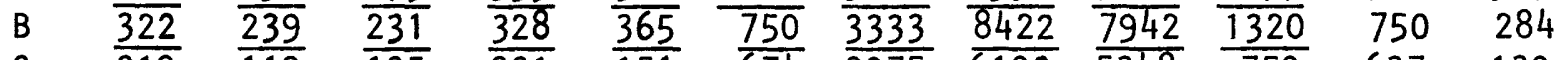

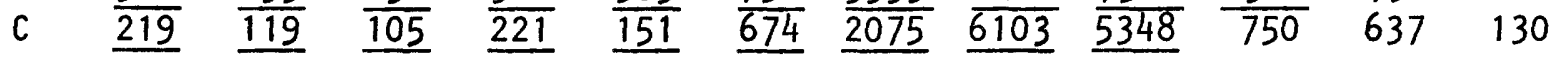

WITH TRANSMOUNTAIN DIVERSIONS

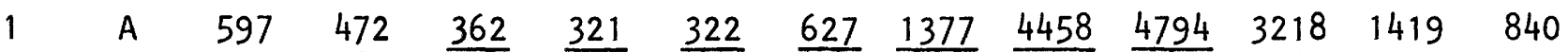

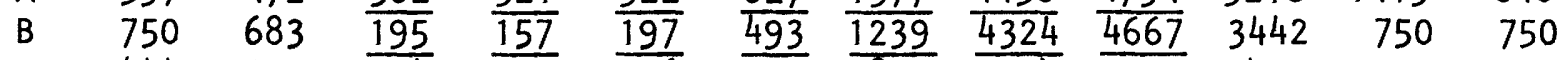

$\begin{array}{llllllllllllll}\text { C } 444 & 111 & \underline{104} & \underline{101} & \underline{116} & \underline{301} & \underline{857} & \underline{3324} & \underline{3501} & 2435 & 750 & 750\end{array}$

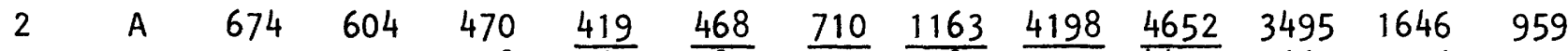

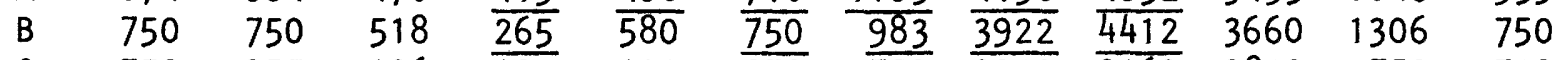

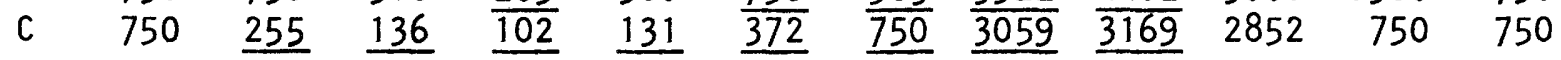

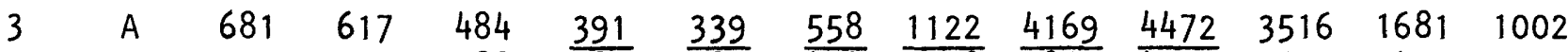

$\begin{array}{lllllllllllll}\text { B } & 750 & 750 & 588 & \frac{183}{183} & \frac{382}{182} & \frac{524}{424} & \overline{938} & \overline{3861} & \frac{4297}{360} & 3672 & 1429 & 750\end{array}$

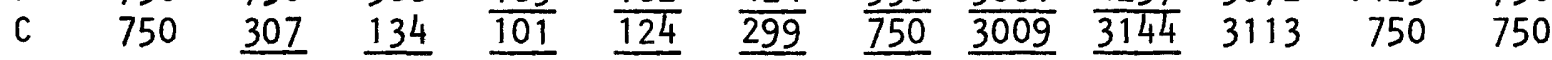

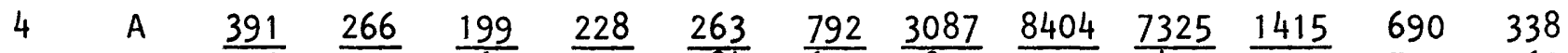

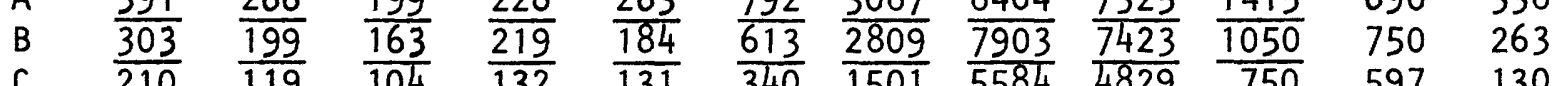

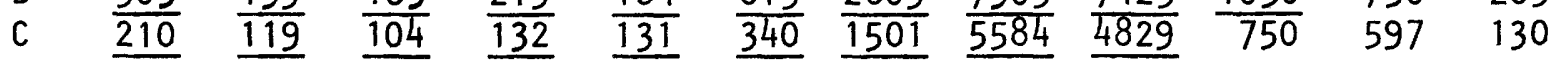


The Yampa River at Deerlodge Park is the last downstream control point in this simulation. As such, this location represents the total effect of all reservoir-development options, transmountain diversions, and water-use allocation on the outflow from the Yampa River basin. An average desired monthly streamflow of $750 \mathrm{ft}^{3} / \mathrm{s}$ was specified at this control point. This was computed from a combination of the $690-\mathrm{ft}^{3} / \mathrm{s}$ flow required by the Colorado River Compact of 1948 at the Maybell streamflow gage (control point 18) and the Little Snake River drainage input. Some consideration also was given to the proposed Wild and Scenic River designation within Dinosaur National Monument (H. J. Belisle, U.S. Bureau of Reclamation, written commun., 1976).

At this downstream point in the Yampa River basin, the streamflow statistics were similar for reservoir-development options 1, 2, and 3. Because of the size of the upstream Juniper and Cross Mountain Reservoirs and the other smaller proposed upstream reservoirs (table 2) and also because of the desired flow requirement at this location, the flow statistics indicated large regulated impacts for monthly flow values (tables 48 through 51). The desired flow of $750 \mathrm{ft}^{3} / \mathrm{s}$ was maintained throughout reservoir-development options 1,2 , and 3 for water-use allocations at the 25- and 50-percent levels (tables 48 and 49). Several values less than the $750-\mathrm{ft}^{3} / \mathrm{s}$ desired flow can be seen in tables 50 and 51 and at the 75- and 100-percent water-use allocation. A slight decline in flow was also noticed with progression from reservoir-development options 1, 2, and 3, due to increased proposed reservoir development upstream in the basin. Option 4 without Juniper and Cross Mountain Reservoirs reflected a fairly traditional annual hydrograph with slightly reduced levels of flow and appeared least affected by the water-use allocation percentage increases (tables 48 through 51). Because of the more traditional annual hydrograph (table 47), reservoir-development option 4, as well as the historical conditions, meets the hypothetical $750-\mathrm{ft}^{3} / \mathrm{s}$ desired flow target for 6 or more months each year (tables 48 through 51 ).

The flow statistics with both Vidler and Hog Park transmountain diversions at the 100-percent allocation are presented in table 47. The combined effect can be easily noticed when compared to the historical flow statistics, but when included with reservoir-development options (tables 48 through 51), it has the effect of reducing the number of months that the desired flow is met for both the median and 5-year low-flow (80-percent exceedence) statistics. 


\section{SUMMARY}

A total of 35 major reservoirs has been proposed for construction in the Yampa River basin to provide additional water for increasing industrial, irrigation, and municipal uses. In addition, two transmountain diversions have been proposed to transport water to the metropolitan areas of Denver, Colo., and Cheyenne, Wyo. A multireservoir-flow computer model was used to simulate the effects on streamflow of five potential options, including one representing historical conditions (no additional reservoir development) and four representing various degrees of reservoir and transmountain-diversion development. Various combinations of 17 of the larger proposed reservoirs and the 2 transmountain diversions were used in the model analysis.

Simulated monthly historical streamflows at 47 control points throughout the Yampa River basin were determined for the following conditions:

A. Historical conditions:

1. Historical conditions wi thout any proposed diversions.

2. Historical conditions with 100 percent of proposed transmountain diversions.

B. Reservoir-development options 1 through 4 :

1. Allocation of 25 percent of total active reservoir storage for agricultural use without any transmountain diversions, and including 100 percent of industrial and municipal diversions.

2. Allocation of 25 percent of total active reservoir storage for agricultural use with 25 percent of proposed transmountain diversions, and including 100 percent of industrial and municipal diversions.

3. Allocation of 50 percent of total active reservoir storage for agricultural use wi thout any transmountain diversions, and including 100 percent of industrial and municipal diversions.

4. Allocation of 50 percent of total active reservoir storage for agricultural use with 50 percent of proposed transmountain diversions, and including 100 percent of industrial and municipal diversions.

5. Allocation of 75 percent of total active reservoir storage for agricultural use without any transmountain diversions, and including 100 percent of industrial and municipal diversions.

6. Allocation of 75 percent of total active reservoir storage for agricultural use with 75 percent of proposed transmountain diversions, and including 100 percent of industrial and municipal diversions.

7. Allocation of 100 percent of total active reservoir storage for agricultural use without any transmountain diversions, and including 100 percent of industrial and municipal diversions.

8. Allocation of 100 percent of total active reservoir storage for agricultural use with 100 percent of proposed transmountain diversions, and including 100 percent of industrial and municipal diversions. 
Results of this study were designed to demonstrate the application of a reservoir-modeling computer technique in helping to evaluate certain areas of concern for proposed reservoir development in the Yampa River basin. This study, as presented, is an extension of earlier reservoir-modeling work completed for the Yampa River basin (Adams and others, 1982). Results presented in this report are somewhat speculative because of assumptions which had to be made for model application and also because of the limited possible reservoir-development schemes considered for the study. The results, however, do present some possible impacts of the proposed sequential reservoir development in the Yampa River basin.

By varying the percentages of agricultural and transmountain diversions within each proposed reservoir-development option, lesser degrees of development than those currently (1979) proposed were simulated, thus providing results for a greater range of alternatives. The results of these simulations will provide water managers and planners with some insight on how proposed surface-water developments will affect minimum streamflows.

During partial model verification for the basin, the fit of the mean monthly streamflow statistics to streamflow-gaging-station records statistics was good to fair ( 5 to 20 percent). Comparisons between the main-stem Yampa River model results and streamflow-gaging-station record statistics show a decrease in model accuracy in the downstream direction from a minus 5-percent difference at Steamboat Springs to a minus 20-percent difference at the Craig or Maybell streamflow-gaging stations.

Model-simulation results for nine representative control points presented in this report are summarized below. Results for the remaining 38 control points may be obtained from the U.S. Geological Survey for the cost of computer and reproduction time.

For certain tributary locations--namely the Elk River near Trull (control point 38) and Trout Creek at mouth (control point 34)--the monthly flow statistics are far less affected by allocation percentages or development option than are those for the Yampa River main stem. Because some of these reservoirs were only operating at minimal storage levels during most of the period of record and could not fulfill their corresponding diversion allocations, the effects of these reservoirs on streamflow are minor. The transmountain diversions also have little effect at these two locations. In general, the 50-year monthly flow statistics for any tributary to the Yampa River exhibit regulated flow patterns only if that tributary had one or more reservoirs upstream.

Yampa River main-stem sites responded in different ways, depending on their location in the proposed reservoir system and other downstream and upstream demands. In general, all locations studied responded to increases in agriculturaldiversion water-use allocation percentage and transmountain diversion with reduction in streamflow. In some instances, streamflow in certain reaches could be increased by releases from upstream reservoirs resulting from downstream reservoir demands. 
The Vidler and Hog Park transmountain diversions had noticeable effects on most Yampa River main-stem sites. The Vidler transmountain diversion could affect all Yampa River locations downstream from Steamboat Springs, Colo., while the Hog Park diversion could affect all Little Snake River locations downstream from approximately Dixon, Wyo. Both diversions could affect the Yampa River near Deerlodge Park. The Steamboat Springs location is most highly affected by the Vidler transmountain diversion with lesser impact downstream.

of the nine sites of interest presented in this report, three of the sites had some periods of projected zero flow for the 50-year monthly-flow simulation. The Steamboat Springs location had periods of zero flow when the Vidler transmountain diversion was included for reservoir-development options 3 and 4 at the proposed 25-percent water-use allocation and for alloptions at the 100-percent water-use allocation. The Craig location on the Yampa River had some zero-flow periods in reservoir-development option 4 at the 25-percent water-use allocation level, increasing to several instances of zero flow for all options at the 100percent water-use allocation, both with and without the Vidler transmountain diversion. The Yampa River at Maybell maintained some flow until the irrigation water reached 75-percent water-use allocation. At this 75-percent water-use allocation, several zero-flow months occurred, especially during the low-flow season of summer and fall.

\section{SELECTED REFERENCES}

Adams, D. B., Bauer, D. P., Dale, R. H., and Steele, T. D., 1982, Reservoirdevelopment impacts on surface-water quantity and quality in the Yampa River basin, Colorado and Wyoming: U.S. Geological Survey Water-Resources Investigation 31-30 (in press).

Banner \& Associates, Inc., 1976, Proposed expansion of Cheyenne's Little Snake diversion facilities: First section of general report and reconnaissance report on Hog Park Reservoir enlargement, prepared for the City of Cheyenne, Wyo., Board of Public Utilities, 6 chaps. with 3 apps.

Colorado River Water Conservation District, 1975, Federal Power Commission application for preliminary permit, Juniper-Cross Mountain project: Water-development proposal and supporting material, 7 p., app.

Ficke, J. F., Adams, D. B., and Danielson, T. W., 1976, Evaporation for seven reservoirs in the Denver water-supply system, central Colorado: U.S. Geological Survey Water-Resources Investigations 76-114, 170 p.; available only from U.S. Department of Commerce, National Technical Information Service, Springfield, VA 22161 , as report PB-265-323.

Gray, S. L., McKean, J. R., and Weker, J. C., 1977, The economy of northwestern Colorado--Description and analysis: Final report submitted to U.S. Bureau of Land Management, Contract 52500-CT5-1019, 4 chaps., app.

Knudsen, W. I., Jr., and Danielson, J. A., 1977, A discussion of legal and institutional constraints on energy-related water development in the Yampa River basin, northwestern Colorado: U.S. Geological Survey, Contract No. 14-080001-15075, December 1977, 20 p.

Oak Creek Power Company, 1976, Oak Creek Water and Power Project, Colorado: Denver, Van Sickle \& Associates, Inc., Consulting Engineers, January 1976, 22 p. 
Palmer, R. N., James, I. C., II, and Hirsch, R. M., 1977, Comparative assessment of water use and environmental implications of coal slurry pipelines: U.S. Geological Survey Open-File Report 77-698, 29 p.; also published in Hydrological Sciences Bulletin, v. 23, no. 4, December 1976, p. 455-469.

Steele, T. D., 1976, Coal-resource development alternatives, residuals management, and impacts on the water resources of the Yampa River basin: Colorado and Wyoming: International Water Resources Association Symposium on Water Resources and Fossil Fuel Production, Dusseldorf, Germany, September 1976, Paper 28, $17 \mathrm{p}$.

Steele, T. D., Bauer, D. P., Wentz, D. A., and Warner, J. W., 1979, The Yampa River basin, Colorado and Wyoming--A preview to expanded coal-resource development and its impacts on regional water resources: U.S. Geological Survey Water-Resources Investigations 78-126, 133 p.; available only from U.S. Department of Commerce, National Technical Information Service, Springfield, VA 22161 , as report PB-303 815/AS.

U.S. Army, Corps of Engineers, 1968, HEC-3, Reservoir systems analysis: Hydrological Engineering Center users manual no. 23-53, 86 p.

U.S. Department of the Interior, 1976, Savery-Pot Hook project, Colorado and Wyoming--Draft environmental statement: U.S. Bureau of Reclamation, Upper Colorado Region, Salt Lake City, Utah, INT DES 76-37, September 27, 1976, 9 chaps., with attachments.

Western Engineers, Inc., 1975, Yamcolo Reservoir project--Feasibility report: Grand Junction, Prepared for the Upper Yampa Water Conservancy District and the Colorado Water Conservation Board, November 1975, 8 chaps.

Woodward-Clyde Consultants, 1977, Supplemental report to the U.S. Environmental Protection Agency of Lake Catamount Dam and Reservoir: Report for Pleasant Valley Investment Co., Steamboat Springs, Colo., No. 18914-16319, 58 p., app. 

